\title{
One-skeleton galleries, the path model and a generalization of Macdonald's formula for Hall-Littlewood polynomials
}

\author{
Stéphane Gaussent and Peter Littelmann
}

November 8, 2018

Institut Élie Cartan U.M.R. 7502, Université Henri Poincaré Nancy 1, Boîte Postale 70239, F-54506 Vandouvre-lès-Nancy CEDEX, France, and

Mathematisches Institut, Universität zu Köln, Weyertal 86-90, D-50931 Köln, Germany

\begin{abstract}
We give a direct geometric interpretation of the path model using galleries in the 1 -skeleton of the Bruhat-Tits building associated to a semi-simple algebraic group. This interpretation allows us to compute the coefficients of the expansion of the Hall-Littlewood polynomials in the monomial basis. The formula we obtain is a "geometric compression" of the one proved by Schwer, its specialization to the case $\mathrm{A}_{n}$ turns out to be equivalent to Macdonald's formula.
\end{abstract}

\section{Contents}

1 Introduction $\quad 2$

2 Preliminaries $\quad 5$

2.1 Schubert varieties in the affine Grassmannian . . . . . . . . . . . . . 5

2.2 Reduction to the simply connected case . . . . . . . . . . . . 6

2.3 Affine Kac-Moody groups . . . . . . . . . . . . . . . . . . 6

2.4 Hall-Littlewood polynomials . . . . . . . . . . . . . . . . . 7

3 Apartments, chambers and buildings

3.1 Apartment ............................... 8

3.2 Chambers, alcoves, faces and sectors . . . . . . . . . . . . . 8

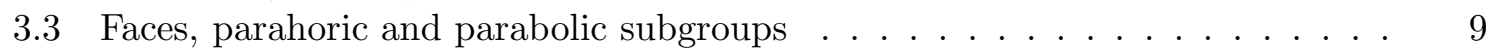

3.4 The affine building . . . . . . . . . . . . . . . . . . 10

3.5 Residue building . . . . . . . . . . . . . . . . . . 10

4 One-skeleton galleries 11

4.1 Combinatorial one-skeleton galleries . . . . . . . . . . . . . 11

4.2 Young tableaux and one-skeleton galleries for classical groups of type $\mathrm{A}_{n}, \mathrm{~B}_{n}, \mathrm{C}_{n} 13$

4.3 Varieties of galleries and Bott-Samelson varieties . . . . . . . . . 15

4.4 Cells ............................... 17 
5 Minimal one-skeleton galleries $\quad 20$

5.1 Minimality relative to an equivalence class of sectors . . . . . . . . . . 20

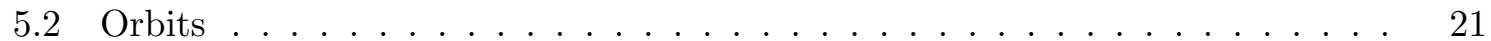

5.3 Positively folded one-skeleton galleries . . . . . . . . . . . . . 22

5.4 Local and global properties in special cases . . . . . . . . . . . . . 23

5.5 Semistandard Young tableaux and positively folded one-skeleton galleries . . 25

6 Local minimality 26

6.1 Positively folded galleries of chambers . . . . . . . . . . . . . . . 26

6.2 Two steps minimal one-skeleton galleries . . . . . . . . . . . . . . . . 28

7 From local properties to global properties

7.1 From positively folded two-steps galleries to minimal galleries . . . . . . . . . 33

$7.2 \quad$ A formula for $L_{\lambda, \mu} \ldots \ldots \ldots \ldots \ldots \ldots \ldots$

8 Dimension of $r_{-\infty}^{\min }(\delta)$, LS-galleries and Young tableaux $\quad \mathbf{3 6}$

8.1 LS one-skeleton galleries . . . . . . . . . . . . . . . . 37

8.2 Reduction to the case of a fundamental weight . . . . . . . . . . 38

8.3 Connection with the path model . . . . . . . . . . . . . . . . . . . . . . . . . . . . .

8.4 LS-tableaux and LS-galleries . . . . . . . . . . . . . . . . 41

\section{Introduction}

We give a direct geometric interpretation of the path model for representations and the associated Weyl group combinatorics [20]. As a consequence, we get a generalization of Macdonald's formula for Hall-Littelwood polynomials in type $A_{n}$ [23. Our formula can be seen as a geometric compression of Schwer's formula [26].

Concerning the connection with the path model, a first step in this direction was done in 9]. The advantage of the new approach is that galleries in the one-skeleton of the apartment can directly be identified with piecewise linear paths running along the one-skeleton. They can be concatenated and they can also be easily translated in the language of tableaux, for classical groups. The goal now is to show that the original approach by Lakshmibai, Musili and Seshadri [14, 16] towards what later became the path model has an intrinsic geometric interpretation in the geometry of the affine Grassmannian, respectively in the geometry of the associated affine building. Another instance of this approach can be found in the work of Kapovich and Millson [10] where they use paths in the one-skeleton in their proof of the "saturation" theorem.

To be more precise, let $G$ be a semisimple algebraic group defined over $\mathbb{C}$, fix a Borel subgroup $B$ and a maximal torus $T$. Let $U^{-}$be the unipotent radical of the opposite Borel subgroup. Let $\mathcal{O}=\mathbb{C}[[t]]$ be the ring of complex formal power series and let $\mathcal{K}=\mathbb{C}((t))$ be the quotient field. For a dominant coweight $\lambda$ and an arbitrary coweight $\mu$ consider the following intersection in the affine Grassmannian $G(\mathcal{K}) / G(\mathcal{O})$ :

$$
Z_{\lambda, \mu}=G(\mathcal{O}) \cdot \lambda \cap U^{-}(\mathcal{K}) \cdot \mu \text {. }
$$

Let $\mathbb{F}_{q}$ be the finite field with $q$ elements and replace the field of complex numbers by the algebraic closure $K$ of $\mathbb{F}_{q}$. Assume that all groups are defined and split over $\mathbb{F}_{q}$. Replace $\mathcal{K}$ by 
$\mathcal{K}_{q}=\mathbb{F}_{q}((t))$ and $\mathcal{O}$ by $\mathcal{O}_{q}=\mathbb{F}_{q}[[t]]$; the Laurent polynomials $L_{\lambda, \mu}$ defined by $L_{\lambda, \mu}(q)=\left|Z_{\lambda, \mu}^{q}\right|$ show up as coefficients in the Hall-Littlewood polynomial: $P_{\lambda}=\sum_{\mu \in X_{+}^{\vee}} q^{-\langle\rho, \lambda+\mu\rangle} L_{\lambda, \mu} m_{\mu}$.

We replace the desingularization of the Schubert variety $X_{\lambda}$ in [9] by a Bott-Samelson type variety $\Sigma$ which is a fibred space having as factors varieties of the form $H / R$, where $H$ is a semisimple algebraic group and $R$ is a maximal parabolic subgroup. In terms of the affine building, a point in this variety is a sequence $\delta=\left(P_{0}=G(\mathcal{O}), Q_{0}, P_{1}, Q_{1}, \ldots, P_{r}, Q_{r}, P_{r+1}\right)$ of parahoric subgroups of $G(\mathcal{K})$ reciprocative contained in each other, i.e. $G(\mathcal{O}) \supset Q_{0} \subset P_{1} \supset$ $Q_{1} \subset \ldots \supset Q_{r} \subset P_{r+1}$. These desingularizations are smaller than the ones used in [9], in the sense that the fibres are of smaller dimensions. In type $\mathrm{A}_{n}$, these coincide with convolution morphisms.

In terms of the faces of the building, a point in $\Sigma$ is a sequence of closed one-dimensional faces (corresponding to the parahoric subgroups $Q_{0}, \ldots, Q_{r}$ ), where successive faces have (at least) a common zero-dimensional face (i.e. a vertex corresponding to one of the maximal parahoric subgroups $\left.P_{0}, \ldots, P_{r+1}\right)$. So if the sequence is contained in an apartment, then the point in $\Sigma$ can be seen as a piecewise linear path in the apartment joining the origin with a special vertex.

We introduce the notion of a minimal one-skeleton gallery (which always lies in some apartment) and of a positively folded combinatorial gallery in the one-skeleton. The points in $\Sigma$ corresponding to the points in the open orbit $G(\mathcal{O}) \cdot \lambda \subset X_{\lambda}$ are exactly the minimal galleries, we identify those two sets. By choosing a generic one parameter subgroup of $T$ in the anti-dominant Weyl chamber, we get a Białynicki-Birula decomposition of $\Sigma$, the centers $\delta$ of the cells $C_{\delta}$ correspond to combinatorial one-skeleton galleries $\delta$ (i.e. the galleries lying in the standard apartment). We show that $C_{\delta} \cap G(\mathcal{O}) \cdot \lambda \neq \emptyset$ if and only if $\delta$ is positively folded.

The Białynicki-Birula decomposition of $\Sigma$ can be used to define a decomposition $Z_{\lambda, \mu}=$ $\bigcup_{\delta} Z_{\lambda, \mu} \cap C_{\delta}$, the indexing set of the strata are positively folded one-skeleton galleries. To see the geometrical compression (compare Lenart [18], 19]), recall the decomposition in [9], $Z_{\lambda, \mu}^{q}=\bigcup S_{\Delta}$, where the $\Delta$ 's are certain galleries of alcoves of a fixed type in the appartment. Now, fix a minimal gallery of alcoves $\Delta_{\lambda}$ between 0 and $\lambda$ and a minimal one-skeleton gallery $\gamma_{\lambda}$ contained in $\Delta_{\lambda}$. This allows to build a map from the galleries of alcoves in the standard apartment to the one-skeleton ones staying in this apartment. This map sends positively folded galleries of alcoves onto positively folded one-skeleton galleries. Note that this application [even when restricted to positively folded galleries] is surjective but not injective. Further, the pieces $S_{\Delta}$ group together to build the pieces $Z_{\lambda, \mu} \cap C_{\delta}$ of our new decomposition of $Z_{\lambda, \mu}$.

For example, in the $\mathrm{A}_{n}$-case the galleries can be translated into the language of Young tableaux, and the positively folded galleries ending in $\mu$ correspond exactly to the semistandard Young tableaux of shape $\lambda$ and weight $\mu$. In this sense, the new decomposition can be viewed as the optimal geometric decomposition for type $\mathrm{A}_{n}$.

To investigate the intersection $Z_{\lambda, \mu} \cap C_{\delta}$ we need to unfold the (possibly) folded gallery $\delta$. As a consequence of the unfolding procedure we present the formula for the coefficients of the Hall-Littlewood polynomials, the summands below counting the number of points in the intersection of $Z_{\lambda, \mu}^{q} \cap C_{\delta}$ for $\delta$ being positively folded and ending in $\mu$ :

Theorem 2 .

$$
L_{\lambda, \mu}(q)=\sum_{\delta \in \Gamma^{+}\left(\gamma_{\lambda}, \mu\right)} q^{\ell\left(w_{D_{0}}\right)} \prod_{j=1}^{r} U_{i}(q),
$$


where $U_{i}(q)$ is a polynomial of the form $\sum_{\mathbf{c} \in \Gamma_{s^{j}}^{+}\left(\mathbf{i}_{j}, o p\right)} q^{t(\mathbf{c})}(q-1)^{r(\mathbf{c})}$ that counts the number of points over $\mathbb{F}_{q}$ in a subvariety of a generalised Grassmannian $H / R$, where $H$ and $R$ are determined by $\delta$.

To get a rough idea of what this formula means without getting drowned by the technical details, let us consider the case where $G$ is of type $\mathrm{A}_{n}$. We identify the positively folded galleries with the semi-standard Young tableaux of shape $\lambda$ having weight $\mu$. We use the convention that the entries in the tableau are weakly increasing in the rows and stricly increasing in the columns, so the one dimensional faces of the gallery correspond to the columns of the tableau. We enumerate the columns such that the right most column is the first one. Given such a tableau $\delta$, let $E_{0}, \ldots, E_{r}$ be the columns. We want to investigate the set of all minimal galleries in $C_{\delta}$ lying in $Z_{\lambda, \mu}$. Proposition 15 shows that this set has a product structure

$$
B^{-} w_{D_{0}} Q_{E_{0}}^{-} / Q_{E_{0}}^{-} \times \prod_{j=1}^{r} \operatorname{Min}\left(E_{j-1}, E_{j}\right),
$$

which explains the product structure for each summand in Theorem 2, To get a minimal gallery in the building that lifts $\delta$, i.e. is an element of $C_{\delta}$ and lies in $Z_{\lambda, \mu}$, the possibilities for the first column $E_{0}$ form a Schubert cell leading to the term $q^{\ell\left(w_{D_{0}}\right)}$. For $j \geq 1$, the possibilities for lifts of $E_{j}$ depend on the column $E_{j-1}$ before. It can be shown that Macdonald's algorithm (see [23]) can be expressed also columnwise. More precisely, Klostermann [12 has shown in the framework of her thesis that the structure of the second sum in the formula above in Theorem 2 can be simplified in the $\mathrm{A}_{n}$-case so that, in terms of Young tableaux, the resulting algorithm is exactly the same as Macdonald's algorithm.

The positively folded one-skeleton galleries having $q^{\langle\lambda+\mu, \rho\rangle}$ as a leading term in the counting formula for $\left|Z_{\lambda, \mu}^{q} \cap C_{\delta}\right|$, are called LS-galleries; this is an abbreviation for LakshmibaiSeshadri galleries. As in [9], for an LS-gallery $\delta, \overline{Z_{\lambda, \mu} \cap C_{\delta}}$ is an MV-cycle.

In section 8 we discuss the special role of the LS-galleries and the connection with the indexing system by generalized Young tableaux introduced by Lakshmibai, Musili and Seshadri in a series of papers, see for example [14, 16, 17. Recall that these papers were the background for the path model theory started in [20]. An important notion introduced in the theory of standard monomials is the defining chain ([14, 16, see also section 5), which was a breakthrough on the way for the definition of standard monomials and generalized Young tableaux. In the context of the crystal structure of the path theory this notion again turned up to be an important combinatorial tool to check whether a concatenation of paths is in the Cartan component or not. Still, the definition had the air of an ad hoc combinatorial tool. But in the context of Białynicki-Birula cells, the folding of a minimal gallery by the action of the torus occurs naturally: during the limit process (going to the center of the cell) the direction (= the sector, see section 51) attached to a minimal gallery is transformed into the weakly decreasing sequence of Weyl group elements, the defining chain for the positively folded one-skeleton gallery in the center of the cell.

The connection between the path model theory and the one-skeleton galleries is summarized in the following corollary. For a fundamental coweight $\omega$ let $\pi_{\omega_{i}}:[0,1] \rightarrow X_{\mathbb{R}}^{\vee}, t \mapsto t \omega$ be the path which is just the straight line joining $\mathfrak{o}$ with $\omega$ and let $\gamma_{\omega}$ be the one-skeleton gallery obtained as the sequence of edges and vertices lying on the path (see also Example 1 in Section 4.1). 
Corollary 3. Write a dominant coweight $\lambda=\omega_{i_{1}}+\ldots+\omega_{i_{r}}$ as a sum of fundamental coweights, write $\underline{\lambda}$ for this ordered decomposition. Let $\mathcal{P}_{\underline{\lambda}}$ be the associated path model of LS-paths of shape $\underline{\lambda}$ defined in [20] having as starting path the concatenation $\pi_{\omega_{i_{1}}} * \ldots * \pi_{\omega_{i_{r}}}$. For a path $\pi$ in the path model denote by $\gamma_{\pi}$ the associated gallery in the one-skeleton of $\mathbb{A}$ obtained as the sequence of edges and vertices lying on the path. The one-skeleton galleries $\gamma_{\pi}$ obtained in this way are precisely the LS-galleries of the same type as $\gamma_{\omega_{i_{1}}} * \ldots * \gamma_{\omega_{i_{r}}}$.

In fact, the notion of a defining chain for LS-paths coincides in this case with the notion of a defining chain for the associated gallery.

Since the number of the LS-galleries is the coefficient of the leading term of $L_{\lambda, \mu}$, and since $P_{\lambda} \rightarrow s_{\lambda}$ for $q \rightarrow \infty$, we get as an immediate consequence of Theorem 2 the following character formula. In combination with Corollary [3, this provides a geometric proof of the path character formula, first conjectured by Lakshmibai (see for example [17]) and proved in 20]:

Corollary 4, Char $V(\lambda)=\sum_{\delta} e^{\text {target }(\delta)}$, where the sum runs over all LS-galleries of the same type as $\gamma_{\lambda}$.

The article is organized as follows: In section 2 we recall some basic facts about the affine Grassmannian and Hall-Littlewood polynomials, in section 3 we recall the main facts from building theory needed later. In section 4, we introduce the main object of this article, the one-skeleton galleries of a fixed type, and its geometric counterpart, the Bott-Samelson variety $\Sigma$. We give a description of the Białynicki-Birula cells of $\Sigma$. For groups of type $\mathrm{A}_{n}, \mathrm{~B}_{n}, \mathrm{C}_{n}$, we establish a bijection between galleries and tableaux. In section 5 we introduce the notion of a minimal one-skeleton gallery and of a positively folded combinatorial gallery in the one-skeleton. We show that the correspondence between galleries and tableaux restricts to a bijection between positively folded galleries and semistandard tableaux. In section 6 we unfold the folded galleries locally, in section 7 we do this stepwise for the full gallery and we prove: a cell $C_{\delta}$ contains minimal galleries if and only if $\delta$ is positively folded. In subsection 7.2 we present the formula for the coefficients of the Hall-Littlewood polynomials. In section 8 we discuss the special role of the LS-galleries and the connection with the indexing system by generalized Young tableaux introduced by Lakshmibai, Musili and Seshadri.

\section{Preliminaries}

Let $G$ be a connected complex semisimple algebraic group, we fix a Borel subgroup $B \subset G$ and a maximal torus $T \subset B$. Let $\mathcal{O}=\mathbb{C}[[t]]$ be the ring of complex formal power series and let $\mathcal{K}=\mathbb{C}((t))$ be the quotient field. Denote by $v: \mathcal{K}^{*} \rightarrow \mathbb{Z}$ the standard valuation such that $\mathcal{O}=\{f \in \mathcal{K} \mid v(f) \geq 0\}$. As a set, the affine Grassmannian $\mathcal{G}$ is the quotient

$$
\mathcal{G}=G(\mathcal{K}) / G(\mathcal{O})
$$

Note that $G(\mathcal{K})$ and $\mathcal{G}$ are $i n d$-schemes and $G(\mathcal{O})$ is a group scheme $([13])$. The $G(\mathcal{O})$-orbits in $\mathcal{G}$ are finite dimensional quasi-projective varieties.

\subsection{Schubert varieties in the affine Grassmannian}

We recall the classification of $G(\mathcal{O})$-orbits and the associated $G(\mathcal{O})$-stable Schubert varieties. Denote by $\langle\cdot, \cdot\rangle$ the non-degenerate pairing between the character group $X:=\operatorname{Mor}\left(T, \mathbb{C}^{*}\right)$ of 
$T$ and its group $X^{\vee}:=$ Mor $\left(\mathbb{C}^{*}, T\right)$ of cocharacters. Let $\Phi \subset X$ be the root system of the pair $(G, T)$, and, corresponding to the choice of $B$, denote $\Phi^{+}$the set of positive roots, let $\Delta=\left\{\alpha_{1}, \ldots, \alpha_{n}\right\}$ be the set of simple roots, and let $\rho$ be half the sum of the positive roots.

Let $\Phi^{\vee} \subset X^{\vee}$ be the dual root system, together with a bijection $\Delta \rightarrow \Delta^{\vee}, \alpha \mapsto \alpha^{\vee}$. We denote by $R_{+}^{\vee}$ the submonoide of the coroot lattice $R^{\vee}$ generated by the positive coroots $\Phi_{+}^{\vee}$. We define on $X^{\vee}$ a partial order by setting $\lambda \succ \nu \Leftrightarrow \lambda-\nu \in R_{+}^{\vee}$. Let $X_{+}^{\vee}$ be the cone of dominant cocharacters:

$$
X_{+}^{\vee}:=\left\{\lambda \in X^{\vee} \mid\langle\lambda, \alpha\rangle \geq 0 \forall \alpha \in \Phi^{+}\right\} .
$$

Given $\lambda \in X^{\vee}$, we can view in fact $\lambda$ as an element of $G(\mathcal{K})$. By abuse of notation we write also $\lambda$ for the corresponding class in $\mathcal{G}$.

Let $e v: G(\mathcal{O}) \rightarrow G$ be the evaluation maps at $t=0$ and let $\mathcal{B}=e v^{-1}(B)$ be the corresponding Iwahori subgroup. Then

$$
\mathcal{G}=\bigcup_{\lambda \in X^{\vee}} \mathcal{B} \cdot \lambda=\bigcup_{\lambda \in X_{+}^{\vee}} G(\mathcal{O}) \cdot \lambda
$$

We denote by $X(\lambda)=\overline{\mathcal{B} . \lambda}$ the corresponding Schubert variety. Let $N=N_{G}(T)$ be the normalizer in $G$ of the fixed maximal torus $T$, we denote by $W$ the Weyl group $N / T$ of $G$. Note that for $\lambda \in X_{+}^{\vee}$ we have

$$
\overline{G(\mathcal{O}) \cdot \lambda}=X\left(w_{0}(\lambda)\right)
$$

where $w_{0}$ is the longest element in the Weyl group $W$. By abuse of notation we just write $X_{\lambda}$ for the variety $X\left(w_{0}(\lambda)\right)$ of dimension $\langle 2 \lambda, \rho\rangle$.

\subsection{Reduction to the simply connected case}

Let now $p: G^{\prime} \rightarrow G$ be an isogeny with $G^{\prime}$ being simply connected. The natural map $p_{\mathcal{O}}: G^{\prime}(\mathcal{O}) \rightarrow G(\mathcal{O})$ is surjective and has the same kernel as $p$. Let $X^{\prime}$ and $X^{\prime \vee}$ be the character group respectively group of cocharacters of $G^{\prime}$ for a maximal torus $T^{\prime} \subset G^{\prime}$ such that $p\left(T^{\prime}\right)=T$, then $p: T^{\prime} \rightarrow T$ induces an inclusion $X^{\prime \vee} \hookrightarrow X^{\vee}$.

The quotient $X^{\vee} / X^{\prime \vee}$ measures the difference between $\mathcal{G}$ and the affine grassmannian $\mathcal{G}^{\prime}=G^{\prime}(\mathcal{K}) / G^{\prime}(\mathcal{O})$. In fact, $\mathcal{G}^{\prime}$ is connected, and the connected components of $\mathcal{G}$ are indexed by $X^{\vee} / X^{\prime \vee}$. The natural maps $p_{\mathcal{K}}: G^{\prime}(\mathcal{K}) \rightarrow G(\mathcal{K})$ and $p_{\mathcal{O}}: G^{\prime}(\mathcal{O}) \rightarrow G(\mathcal{O})$ induce a $G^{\prime}(\mathcal{K})$-equivariant inclusion $\mathcal{G}^{\prime} \hookrightarrow \mathcal{G}$, which is an isomorphism onto the component of $\mathcal{G}$ containing the class of 1 . Now $G^{\prime}(\mathcal{K})$ acts via $p_{\mathcal{K}}$ on all of $\mathcal{G}$, and each connected component is a homogeneous space for $G^{\prime}(\mathcal{K})$, isomorphic to $G^{\prime}(\mathcal{K}) / \mathcal{Q}$ for some parahoric subgroup $\mathcal{Q}$ of $G^{\prime}(\mathcal{K})$ which is conjugate to $G(\mathcal{O})$ by an outer automorphism.

So to study $G(\mathcal{O})$-orbits on $G(\mathcal{K}) / G(\mathcal{O})$ for $G$ semisimple, without loss of generality we may sometimes for convenience assume that $G$ is simply connected, but we have to investigate more generally $G(\mathcal{O})$-orbits on $G(\mathcal{K}) / \mathcal{Q}$ for all parahoric subgroups $\mathcal{Q} \subset G(\mathcal{K})$ conjugate to $G(\mathcal{O})$ by an outer automorphism.

\subsection{Affine Kac-Moody groups}

In the following let $G$ be a simply connected semisimple complex algebraic group. The rotation operation $\gamma: \mathbb{C}^{*} \rightarrow \operatorname{Aut}(\mathcal{K}), \gamma(z)(f(t))=f(z t)$ gives rise to group automorphisms $\gamma_{G}: \mathbb{C}^{*} \rightarrow$ Aut $(G(\mathcal{K}))$, we denote $\mathcal{L}(G(\mathcal{K}))$ the semidirect product $\mathbb{C}^{*} \ltimes G(\mathcal{K})$. The rotation operation on 
$\mathcal{K}$ restricts to an operation on $\mathcal{O}$ and hence we have a natural subgroup $\mathcal{L}(G(\mathcal{O})):=\mathbb{C}^{*} \ltimes G(\mathcal{O})$ (for this and the following see [13], Chapter 13).

Let $\hat{\mathcal{L}}(G)$ be the affine Kac-Moody group associated to the affine Kac-Moody algebra

$$
\hat{\mathcal{L}}(\mathfrak{g})=\mathfrak{g} \otimes \mathcal{K} \oplus \mathbb{C} c \oplus \mathbb{C} d,
$$

where $0 \rightarrow \mathbb{C} c \rightarrow \mathfrak{g} \otimes \mathcal{K} \oplus \mathbb{C} c \rightarrow \mathfrak{g} \otimes \mathcal{K} \rightarrow 0$ is the universal central extension of the loop algebra $\mathfrak{g} \otimes \mathcal{K}$ and $d$ denotes the scaling element. We have corresponding exact sequences also on the level of groups, i.e., $\hat{\mathcal{L}}(G)$ is a central extension of $\mathcal{L}(G(\mathcal{K}))$ :

$$
1 \rightarrow \mathbb{C}^{*} \rightarrow \hat{\mathcal{L}}(G) \stackrel{\pi}{\longrightarrow} \mathcal{L}(G(\mathcal{K})) \rightarrow 1 .
$$

Denote $\mathcal{P}_{\mathcal{O}} \subset \hat{\mathcal{L}}(G)$ the "parabolic" subgroup $\pi^{-1}(\mathcal{L}(G(\mathcal{O})))$, then

$$
\mathcal{G}=G(\mathcal{K}) / G(\mathcal{O})=\mathcal{L}(G(\mathcal{K})) / \mathcal{L}(G(\mathcal{O}))=\hat{\mathcal{L}}(G) / \mathcal{P}_{\mathcal{O}}
$$

Let $N_{\mathcal{K}}$ be the subgroup of $G(\mathcal{K})$ generated by $N$ and $T(\mathcal{K})$, let $\mathcal{T} \subset \hat{\mathcal{L}}(G)$ be the corresponding standard maximal torus (i.e. $\pi(\mathcal{T}) \supset \mathbb{C}^{*} \ltimes T$ ) and let $\mathcal{N}$ be its normalizer in $\hat{\mathcal{L}}(G)$. We get two incarnations of the affine Weyl group:

$$
W^{\mathfrak{a}}=N_{\mathcal{K}} / T=\mathcal{N} / \mathcal{T}
$$

So to study $G(\mathcal{O})$-orbits on $G(\mathcal{K}) / G(\mathcal{O})$ for $G$ semisimple, without loss of generality we may assume that $G$ is simply connected and study $\mathcal{P}_{\mathcal{O}}$-orbits in $\hat{\mathcal{L}}(G) / \mathcal{Q}$, where $\mathcal{Q}$ is a parabolic subgroup of the affine Kac-Moody group $\hat{\mathcal{L}}(G)$ conjugate to $\mathcal{P}_{\mathcal{O}}$ by an outer automorphism.

\subsection{Hall-Littlewood polynomials}

There is a natural action of $W$ on the group algebra $R\left[X^{\vee}\right]$ with coefficients in some ring $R$. For $\mu \in X^{\vee}$ we denote the corresponding basis element by $x^{\mu}$. The algebra of symmetric polynomials $R\left[X^{\vee}\right]^{W}$ is the algebra of invariants under this action. There are several classical bases known for $R\left[X^{\vee}\right]^{W}$, all indexed by dominant coweights. Two important ones are the monomial symmetric polynomials $\left\{m_{\lambda}\right\}_{\lambda \in X_{+}^{\vee}}$ and the Schur polynomials $\left\{s_{\lambda}\right\}_{\lambda \in X_{+}^{\vee}}$. The monomial polynomials are just the orbit sums $m_{\lambda}=\sum_{\mu \in W \lambda} x^{\mu}$. The Schur polynomial $s_{\lambda}$ is the character of the irreducible representation $V(\lambda)$ of the Lie algebra $\mathfrak{g}^{\vee}$ of the Langlands' dual group $G^{\vee}$ of $G$.

Specializing the ring of coefficients $R$ to the $\operatorname{ring} \mathcal{L}:=\mathbb{Z}\left[q, q^{-1}\right]$ of Laurent polynomials we have another basis for $\mathcal{L}\left[X^{\vee}\right]^{W}$, the Hall-Littlewood polynomials $\left\{P_{\lambda}\right\}_{\lambda \in X_{+}^{\vee}}$. They are defined by

$$
P_{\lambda}=\frac{1}{W_{\lambda}\left(q^{-1}\right)} \sum_{w \in W} w\left(x^{\lambda} \prod_{\alpha \in \Phi^{+}} \frac{1-q^{-1} x^{-\alpha^{\vee}}}{1-x^{-\alpha^{\vee}}}\right)
$$

where $W_{\lambda} \subset W$ is the stabilizer of $\lambda$ and $W_{\lambda}(t)=\sum_{w \in W_{\lambda}} t^{\ell(w)}$. The Hall- Littlewood polynomials interpolate between the monomial symmetric polynomials and the Schur polynomials because $P_{\lambda}(1)=m_{\lambda}$ and $P_{\lambda} \rightarrow s_{\lambda}$ for $q \rightarrow \infty$.

We define Laurent polynomials $L_{\lambda, \mu}$ for $\lambda, \mu \in X_{+}^{\vee}$ by

$$
P_{\lambda}=\sum_{\mu \in X_{+}^{\vee}} q^{-\langle\rho, \lambda+\mu\rangle} L_{\lambda, \mu} m_{\mu}
$$


Since $P_{\lambda} \rightarrow s_{\lambda}$ for $q \rightarrow \infty$, we know that $q^{-\langle\rho, \lambda+\mu\rangle} L_{\lambda, \mu} \in \mathbb{Z}\left[q^{-1}\right]$.

The Hall-Littlewood polynomials are connected with the geometry of the affine Grassmannian. Let $B^{-} \subset G$ be the opposite Borel subgroup and denote by $U^{-}$its unipotent radical. We are interested in the structure of the irreducible components of the intersection of the following orbits in $\mathcal{G}$ :

$$
Z_{\lambda, \mu}:=G(\mathcal{O}) \cdot \lambda \cap U^{-}(\mathcal{K}) . \mu \subset \mathcal{G}, \quad \lambda \in X_{+}^{\vee}, \mu \in X^{\vee} .
$$

For a prime power $q$ let $\mathbb{F}_{q}$ be the finite field with $q$ elements, set $\mathcal{K}_{q}:=\mathbb{F}_{q}((t))$ and $\mathcal{O}_{q}:=$ $\mathbb{F}_{q}[[t]]$, and let $Z_{\lambda, \mu}^{q}$ be defined as above, only $\mathcal{K}$ and $\mathcal{O}$ being replaced by $\mathcal{K}_{q}$ and $\mathcal{O}_{q}$. The Laurent polynomials $L_{\lambda, \mu}$ have the following geometric interpretation coming from the Satake isomorphism.

Fact. $\left|Z_{\lambda, \mu}^{q}\right|=L_{\lambda, \mu}(q)$.

\section{Apartments, chambers and buildings}

Instead of studying directly the intersection $Z_{\lambda, \mu}$ in (2), we replace the Schubert variety $X_{\lambda}$ by a desingularization given by an appropriately chosen Bott-Samelson variety or variety of galleries. In this context the $U^{-}(\mathcal{K})$-orbits are replaced by Białynicki-Birula cells associated to a generic anti-dominant coweight. To describe the choice of the desingularization and get hold of the combinatorial tools to calculate $\left|Z_{\lambda, \mu}^{q}\right|$, we need to recall some notation from the theory of buildings. As references we suggest [4], [5], [25] and/or [29].

\subsection{Apartment}

The apartment associated to the root and coroot datum is the real vector space $\mathbb{A}=X^{\vee} \otimes_{\mathbb{Z}} \mathbb{R}$ together with the hyperplane arrangement defined by the set $\{(\alpha, n) \mid \alpha \in \Phi, n \in \mathbb{Z}\}$ of affine roots. In terms of affine Kac-Moody algebras, a couple $(\alpha, n)$ corresponds to the real affine root $\alpha+n \delta$, where $\delta$ denotes the smallest positive imaginary root. For an affine root $(\alpha, n)$ we write $s_{\alpha, n}: x \mapsto x-(\langle\alpha, x\rangle+n) \alpha^{\vee}$ for the affine reflection and $\mathrm{H}_{\alpha, n}=\{x \in \mathbb{A} \mid\langle\alpha, x\rangle+n=0\}$ for the corresponding affine hyperplane of fixed points, and we write

$$
\mathrm{H}_{\alpha, n}^{+}=\{x \in \mathbb{A} \mid\langle\alpha, x\rangle+n \geqslant 0\}
$$

for the corresponding closed half-space. Similarly we define the negative half space $\mathrm{H}_{\alpha, n}^{-}$.

\subsection{Chambers, alcoves, faces and sectors}

Definition 1. The irreducible components of $\mathbb{A}-\bigcup_{\alpha \in \Phi^{+}} \mathrm{H}_{\alpha, 0}$ are called open (spherical) chambers, the closure is called a closed chamber or Weyl chamber, or just chamber. The irreducible components of $\mathbb{A}-\bigcup_{(\alpha, n) \in \Phi^{+} \times \mathbb{Z}} \mathrm{H}_{\alpha, n}$ are called open alcoves, the closure is called a closed alcove or just an alcove.

The Weyl group $W$ and the affine Weyl group $W^{\mathfrak{a}}$ can be realized in this context as follows: $W$ is the finite subgroup of $G L(\mathbb{A})$ generated by the reflections $s_{\alpha, 0}, \alpha \in \Phi$, the affine Weyl group $W^{\mathfrak{a}}$ is the group of affine transformations of $\mathbb{A}$ generated by the affine reflections $s_{\alpha, n}$, $(\alpha, n) \in \Phi \times \mathbb{Z}$. The dominant Weyl chamber

$$
C^{+}:=\left\{x \in \mathbb{A} \mid \forall \alpha \in \Phi^{+}:\langle\alpha, x\rangle \geqslant 0\right\}=\bigcap_{\alpha \in \Phi^{+}} \mathrm{H}_{\alpha, 0}^{+}
$$


is a fundamental domain for the action of $W$ on $\mathbb{A}$ and the fundamental alcove

$$
\Delta_{f}=\left\{x \in \mathbb{A} \mid \forall \alpha \in \Phi^{+}: 0 \leq\langle\alpha, x\rangle \leq 1\right\}=\bigcap_{\alpha \in \Phi^{+}} \mathrm{H}_{\alpha, 0}^{+} \cap \bigcap_{\alpha \in \Phi, n>0} \mathrm{H}_{\alpha, n}^{+}
$$

is a fundamental domain for the action of $W^{\mathfrak{a}}$ on $\mathbb{A}$.

Definition 2. By a face $F$ we mean a subset of $\mathbb{A}$ obtained as the intersection $\bigcap_{(\beta, m)} \mathrm{H}_{\beta, n}^{\bullet}$, where for each pair $(\beta, n), \beta \in \Phi^{+}, n \in \mathbb{Z}$, one choses $\mathrm{H}_{\beta, n}^{\bullet}$ to be either the hyperplane, the positive or the negative halfspace. By the corresponding open face $F^{o}$ we mean the subset of $F$ obtained when replacing the closed affine halfspaces in the definition of $F$ by the corresponding open affine halfspaces.

We call the affine span $\left\langle F^{o}\right\rangle_{\text {aff }}=\langle F\rangle_{\text {aff }}$ the support of the (open) face, the dimension of the face is the dimension of its support. A wall of an alcove is the support of a codimension one face. In general, instead of the term hyperplane we use often the term wall, which is more common in the language of buildings.

For any subset $\Omega$ and any face $F$ contained in an apartment $A$ of a building, we say that a wall $\mathrm{H}$ separates $\Omega$ and $F$ if $\Omega$ is contained in a closed half space defined by $\mathrm{H}$ and $F^{o}$ is a subset of the opposite open half space.

We call a face of dimension one in $\mathbb{A}$ an edge and a face of dimension zero a vertex. For a vertex $\nu$ let $\Phi_{\nu} \subset \Phi$ be the subrootsystem consisting of all roots $\alpha$ such that $\nu \in \mathrm{H}_{(\alpha, n)}$ for some integer $n$. A vertex $\nu$ is called a special vertex if $\Phi_{\nu}=\Phi$. The special vertices are precisely the coweights for $G$ of adjoint type.

By a sector $\mathfrak{s}$ with vertex $\nu \in \mathbb{A}$ we mean a closed chamber translated by $\nu$, i.e., there exists a closed chamber $C$ such that

$$
\mathfrak{s}:=\{\lambda \in \mathbb{A} \mid \lambda=\nu+z \text { for some } z \in C\} .
$$

By abuse of notation we write $-\mathfrak{s}$ for the sector

$$
-\mathfrak{s}=\nu-C=\{\mu \in A \mid \mu=\nu-x \text { for some } x \in C\} .
$$

For a sector $\mathfrak{s}$ with vertex $\nu$ and an element $\mu \in \mathbb{A}$ let $\mathfrak{s}(\mu)$ be the sector obtained from $\mathfrak{s}$ by translating the sector by $\mu-\nu$ : If $\mathfrak{s}$ is as in (3), then

$$
\begin{aligned}
\mathfrak{s}(\mu) & =\{\lambda \in \mathbb{A} \mid \lambda=(\mu-\nu)+z \text { for some } z \in \mathfrak{s}\} \\
& =\{\lambda \in \mathbb{A} \mid \lambda=\mu+z \text { for some } z \in C\}
\end{aligned}
$$

If $\mu \in \mathfrak{s}$, then obviously $\mathfrak{s}(\mu) \subset \mathfrak{s} \subset \mathfrak{s}(-\mu)$.

\subsection{Faces, parahoric and parabolic subgroups}

The faces in $\mathbb{A}$ are in bijection with parabolic subgroups of the affine Kac-Moody group $\hat{\mathcal{L}}(G)$ containing $\mathcal{T}$ and parahoric subgroups in $G(\mathcal{K})$ containing $T$.

To a root vector $X_{\alpha} \in \operatorname{Lie} G$, one associates the one-parameter subgroup $U_{\alpha}=\left\{x_{\alpha}(f)=\right.$ $\left.\exp \left(X_{\alpha} \otimes f\right) \mid f \in \mathcal{K}\right\}$ of $G(\mathcal{K})$ (resp. of $\left.\hat{\mathcal{L}}(G)\right)$. If $f=a t^{n}$ for some $a \in \mathbb{C}$ and $n \in \mathbb{Z}$, then, for a fixed $n$, the set $U_{\alpha+n \delta}=\left\{x_{\alpha}\left(a t^{n}\right) \mid a \in \mathbb{C}\right\}$ is a one-parameter subgroup associated to the real affine root $\alpha+n \delta$. 
Definition 3. Given a face $F$, let $\hat{P}_{F}$ be the unique parabolic subgroup of $\hat{\mathcal{L}}(G)$ containing $\mathcal{T}$ and all root subgroups $U_{\alpha+n \delta}$ such that $F \subset \mathrm{H}_{\alpha, n}^{+}$.

Given a face $F$, let $U_{F}$ be the subgroup of $G(\mathcal{K})$ generated by all elements of the form $x_{\alpha}(f)$, where $f \in \mathcal{K}^{*}$ is such that $v(f) \geq n$ and $F \subset \mathrm{H}_{\alpha, n}^{+}$. Let $P_{F}$ be the unique parahoric subgroup of $G(\mathcal{K})$ containing $T$ and $U_{F}$.

For example, if $F$ is a face of the fundamental alcove, then $\mathcal{B} \subset P_{F}$. Indeed, the fundamental alcove itself corresponds to the Iwahori subgroup $\mathcal{B} \subset G(\mathcal{K})$ respectively the fixed Borel subgroup $\hat{B}$ of $\hat{\mathcal{L}}(G)$. The origin corresponds to the parahoric subgroup $G(\mathcal{O}) \subset G(\mathcal{K})$ respectively the parabolic subgroup $\mathcal{P}_{\mathcal{O}} \subset \hat{\mathcal{L}}(G)$.

\subsection{The affine building}

Let $\mathcal{O}=\mathbb{C}[[t]]$ be the ring of complex formal power series and let $\mathcal{K}=\mathbb{C}((t))$ be the quotient field. Let $N=N_{G}(T)$ be the normalizer in $G$ of the fixed maximal torus $T \subset G$, then the Weyl group $W$ of $G$ is isomorphic to $N / T$. For a real number $r$ let $U_{\beta, r} \subset U_{\beta}(\mathcal{K})$ be the unipotent subgroup

$$
U_{\beta, r}=\{1\} \cup\left\{x_{\beta}(f) \mid f \in \mathcal{K}^{*}, v(f) \geq r\right\} .
$$

For a non-empty subset $\Omega \subset \mathbb{A}$ let $\ell_{\beta}(\Omega)=-\inf _{x \in \Omega}\langle\beta, x\rangle$. We attach to $\Omega$ a subgroup of $G(\mathcal{K})$ by setting

$$
U_{\Omega}:=\left\langle U_{\beta, \ell_{\beta}(\Omega)} \mid \beta \in \Phi\right\rangle .
$$

Let $N(\mathcal{K})$ be the subgroup of $G(\mathcal{K})$ generated by $N$ and $T(\mathcal{K})$. To define the affine building $\mathcal{J}^{\mathfrak{a}}$, let $\sim$ be the relation on $G(\mathcal{K}) \times \mathbb{A}$ defined by:

$$
(g, x) \sim(h, y) \quad \text { if } \exists n \in N(\mathcal{K}) \text { such that } n x=y \text { and } g^{-1} h n \in U_{x},
$$

where $U_{x}=U_{\{x\}}$.

Definition 4. The affine building $\mathcal{J}^{\mathfrak{a}}:=G(\mathcal{K}) \times \mathbb{A} / \sim$ associated to $G$ is the quotient of $G(\mathcal{K}) \times \mathbb{A}$ by " $\sim$ ". The building $\mathcal{J}^{\mathfrak{a}}$ comes naturally equipped with a $G(\mathcal{K})$-action $g \cdot(h, y):=$ $(g h, y)$ for $g \in G(\mathcal{K})$ and $(h, y) \in \mathcal{J}^{\mathfrak{a}}$.

The map $\mathbb{A} \rightarrow \mathcal{J}^{\mathfrak{a}}, x \mapsto(1, x)$ is injective and $N(\mathcal{K})$-equivariant, we will identify in the following $\mathbb{A}$ with its image in $\mathcal{J}^{\mathfrak{a}}$. More generally, a subset $A$ of $\mathcal{J}^{\mathfrak{a}}$ is called an apartment if it is of the form $g \mathbb{A}$ for some $g \in G(\mathcal{K})$. We extend in the same way the notion of a face $F$, a sector $\mathfrak{s}$, a chamber $C$ and the notion of a parahoric subgroup $P_{F}$ associated to a face. Moreover, the action of $G(\mathcal{K})$ is such that the subgroup $U_{\alpha+n \delta}$ fixes the halfspace $\mathrm{H}_{\alpha, n}^{+}$; indeed, $x_{\alpha}\left(a t^{n}\right)$ belongs to $U_{x}$, whence, $\left(x_{\alpha}\left(a t^{n}\right), x\right) \sim(1, x)$.

We denote by $r_{-\infty}: \mathcal{J}^{\mathfrak{a}} \rightarrow \mathbb{A}$ the retraction centered at $-\infty$. It is a chamber complex map and the fibers of $r_{-\infty}$ are the $U^{-}(\mathcal{K})$-orbits in $\mathcal{J}^{\mathfrak{a}}$ (see [9] Definition 8 and Proposition 1, or [5] Sections 6,7).

\subsection{Residue building}

Let $V$ be a vertex in $\mathcal{J}^{\mathfrak{a}}$. Let $\mathcal{J}_{V}^{\mathfrak{a}}$ be the set of all faces $F$ in $\mathcal{J}^{\mathfrak{a}}$ such that $F \supset V$. Following Bruhat and Tits in Remark 4.6.35 of [6], one endows $\mathcal{J}_{V}^{\mathfrak{a}}$ with the complex simplicial structure given by the relation $F \subset F^{\prime}$, for two faces containing $V$. Further, let $\mathrm{H}_{V}$ be the connected reductive subgroup of $G$ with root system $\Phi_{V}$. Then, Theorem 4.6 .33 of loc. cit. shows that 
the structure of a spherical building on the set of all parabolic subgroups of $\mathrm{H}_{V}$ is isomorphic to the one on $\mathcal{J}_{V}^{\mathfrak{a}}$.

This isomorphism restricts to any apartment and implies that if $A$ is an apartment in $\mathcal{J}^{\mathfrak{a}}$, then the set $A_{V}$ of all faces $F \supset V$ contained in $A$ is an apartment in $\mathcal{J}_{V}^{\mathfrak{a}}$. The simplicial structure on $A_{V}$ is the one associated to the Coxeter complex given by the spherical group $W_{V}^{v}$. The latter is the subgroup of $W$ generated by the reflections along $\operatorname{Ker}(\alpha)$, for all $\alpha \in \Phi_{V}$.

Notation. The set $\mathcal{J}_{V}^{\mathfrak{a}}$, endowed with this structure, is called the residue building of $\mathcal{J}^{\mathfrak{a}}$ at $V$. The group $\mathrm{H}_{V}$ acts transitively on the set of pairs $\left(C_{V} \subset A_{V}\right)$ of a chamber in an apartment in $\mathcal{J}_{V}^{\mathfrak{a}}$.

For any face $F$ of $\mathcal{J}^{\mathfrak{a}}$ containing $V$, we denote the associated face in $\mathcal{J}_{V}^{\mathfrak{a}}$ by $F_{V}$. Given a sector $\mathfrak{s}=V+C$ in $\mathbb{A}$ with vertex $V$, one associates the chamber $\mathfrak{s}_{V}$ of $\mathbb{A}_{V}$ in the following way: let $\Delta \supset V$ be the unique alcove in $\mathbb{A}$ such that $\Delta^{o} \cap \mathfrak{s}^{o} \neq \emptyset$, then $\mathfrak{s}_{V}:=\Delta_{V}$. By abuse of notation, $-\mathfrak{s}_{V}$ will denote the chamber associated to $(V-C)$. Let $C_{V}^{ \pm}$denote the positive (resp. negative) chamber in $\mathbb{A}_{V}$ associated to $V+C^{ \pm}$. The stabilizers of $C_{V}^{ \pm}$in $\mathrm{H}_{V}$ are opposite Borel subgroups, denoted by $B_{V}^{ \pm}$.

Let now $V \subset F$ be a one-dimensional face containing a vertex in $\mathcal{J}^{\mathfrak{a}}$. Let $P_{V} \supset P_{F}$ be the parahoric subgroups associated to $V$ and $F$, then $P_{V} / P_{F}$ is isomorphic to a Grassmannian $\mathrm{H}_{V} / Q_{F}$ where $Q_{F} \supset B_{V}$ is the maximal parabolic subgroup in $\mathrm{H}_{V}$ associated to the simple root $\alpha_{F}$ defined by the type of $F_{V}$.

\section{One-skeleton galleries}

Roughly speaking, a one-skeleton gallery is a sequence of edges in $\mathcal{J}^{\mathfrak{a}}$, two subsequent ones having a common vertex. A combinatorial one-skeleton gallery is essentially a gallery that stays in the apartment $\mathbb{A}$. We will see that the set of one-skeleton galleries of fixed type inherits in a natural way the structure of a Bott-Samelson variety $\Sigma$ and provides the desired desingularization of the Schubert variety $X_{\lambda}$ (see Proposition 3). The combinatorial oneskeleton galleries correspond precisely to the centers of Białynicki-Birula cells (Section 4.4) for the smooth variety $\Sigma$.

\subsection{Combinatorial one-skeleton galleries}

Definition 5. We call a sequence $\gamma=\left(V_{0} \subset E_{0} \supset V_{1} \subset E_{1} \supset \cdots \supset V_{r} \subset E_{r} \supset V_{r+1}\right)$ of faces in $\mathbb{A}$ a combinatorial one-skeleton gallery if

- the faces $V_{i}, i=0, \ldots, r+1$, are vertices in $\mathbb{A}$;

- the vertex $V_{0}$ (the source of the gallery) and the vertex $V_{r+1}$ (the target of the gallery) are special vertices;

- the faces $E_{i}, i=0, \ldots, r$, are edges in $\mathbb{A}$.

If $\gamma^{\prime}=\left(V_{0}^{\prime} \subset E_{0}^{\prime} \supset \cdots \subset E_{t}^{\prime} \supset V_{t+1}^{\prime}\right)$ is another one-skeleton gallery such that $V_{0}^{\prime}=V_{r+1}$, then one can concatenate the two galleries to get a new one:

$$
\gamma * \gamma^{\prime}=\left(V_{0} \subset E_{0} \supset \cdots \supset V_{r} \subset E_{r} \supset V_{r+1}=V_{0}^{\prime} \subset E_{0}^{\prime} \supset \cdots \subset E_{t}^{\prime} \supset V_{t+1}^{\prime}\right) .
$$


By abuse of notation we often write $\gamma * \gamma^{\prime}$ even if $V_{0}^{\prime} \neq V_{r+1}$. In this case, we mean the concatenation of $\gamma$ with the displaced gallery $\gamma^{\prime}+\left(V_{r+1}-V_{0}^{\prime}\right)$. This construction makes sense since, by assumption, $V_{0}^{\prime}$ and $V_{r+1}$ are special vertices.

Example 1. Suppose $G$ is simple, of adjoint type and $\omega$ is a fundamental coweight. Let $\mathbb{R}_{\geq 0} \omega \subset \mathbb{A}$ be the extremal ray of the dominant Weyl chamber $C^{+}$spanned by $\omega$. Set $V_{0}=\mathfrak{o}$ and let $E_{0}$ be the unique face of dimension one in the intersection of $\mathbb{R}_{\geq 0} \omega$ with the fundamental alcove. If the second vertex $V_{1}$ of $E_{0}$ is different from $\omega$, then let subsequently $E_{i}$ be the unique dimension one face in $\mathbb{R}_{\geq 0} \omega$ (different from $E_{i-1}$ ) having $V_{i}$ as a common vertex with $E_{i-1}$. We obtain a one-skeleton gallery

$$
\gamma_{\omega}=\left(V_{0}=\mathfrak{o} \subset E_{0} \supset V_{1} \subset \cdots \supset V_{r} \subset E_{r} \supset \omega=V_{r+1}\right)
$$

joining $\mathfrak{o}$ with $\omega$. We refer to these kind of galleries as fundamental galleries, the faces $E_{j}$ of such a gallery are called fundamental faces (although, they might not be contained in the fundamental alcove).

Example 2. Let $\lambda$ be an arbitrary dominant coweight. We call a one-skeleton gallery $\gamma=$ $\left(V_{0} \subset E_{0} \supset \cdots \subset E_{r} \supset V_{r+1}\right)$ a dominant combinatorial gallery joining $\mathfrak{o}$ and $\lambda$ along the coweight lattice if $\gamma=\gamma_{\omega_{i_{1}}} * \gamma_{\omega_{i_{2}}} * \cdots * \gamma_{\omega_{i r}}$ is a concatenation of fundamental galleries such that $\sum_{j=1}^{s} \omega_{i_{j}}=\lambda$.

Example 3. If we have fixed an enumeration $\omega_{1}, \ldots, \omega_{n}$ of the fundamental coweights and $\lambda=\sum a_{i} \omega_{i}$, then we write $\gamma_{\underline{\lambda}}$ for the gallery $\gamma_{a_{1} \omega_{1}} * \cdots * \gamma_{a_{n} \omega_{n}}$ joining $\mathfrak{o}$ and $\lambda$.

Example 4. Let $\lambda$ be again an arbitrary dominant coweight. We call a one-skeleton gallery $\gamma=\left(V_{0} \subset E_{0} \supset \cdots \subset E_{r} \supset V_{r+1}\right)$ a dominant combinatorial gallery joining $\mathfrak{o}$ and $\lambda$ if the source is $\mathfrak{o}$, the target is $\lambda$ and all the faces $E_{j}$ are displaced fundamental faces.

Definition 6. Let $\mathcal{S}^{\mathfrak{a}}$ be the set of affine roots $(\alpha, n)$ such that $\Delta_{f} \cap \mathrm{H}_{(\alpha, n)}$ is a face of codimension one. Given a face $F$ of the fundamental alcove $\Delta_{f}$, we call $\mathcal{S}^{\mathfrak{a}}(F):=\{(\alpha, n) \in$ $\left.\mathcal{S}^{\mathfrak{a}} \mid F \subset \mathrm{H}_{(\alpha, n)}\right\}$ the type of $F$. Given an arbitrary face $F \subset \mathbb{A}$, there exists a unique face $F^{f}$ of the fundamental alcove which is $W^{\mathfrak{a}}$-conjugate to $F$. We set $\mathcal{S}^{\mathfrak{a}}(F):=\mathcal{S}^{\mathfrak{a}}\left(F^{f}\right)$ and call this the type of $F$.

Definition 7. Given a combinatorial one-skeleton gallery $\gamma=\left(V_{0} \subset E_{0} \supset V_{1} \subset \cdots \supset V_{r} \subset\right.$ $\left.E_{r} \supset V_{r+1}\right)$, we call the sequence

$$
t_{\gamma}:=\left(\mathcal{S}^{\mathfrak{a}}\left(V_{0}\right) \supset \mathcal{S}^{\mathfrak{a}}\left(E_{0}\right) \subset \mathcal{S}^{\mathfrak{a}}\left(V_{1}\right) \supset \ldots \subset \mathcal{S}^{\mathfrak{a}}\left(V_{r}\right) \supset \mathcal{S}^{\mathfrak{a}}\left(E_{r}\right) \subset \mathcal{S}^{\mathfrak{a}}\left(V_{r+1}\right)\right)
$$

the gallery of types or the type of $\gamma$. We denote by $\Gamma\left(t_{\gamma}, V_{0}\right)$ the set of all combinatorial galleries starting in $V_{0}$ and having $t_{\gamma}$ as type.

Notation. Because a face $F$ is always contained in an apartment $A=g \mathbb{A}$, the notion of a one-skeleton gallery, of the type of a face and the type of a gallery extends to the whole building $\mathcal{J}^{\mathfrak{a}}$.

Let $W_{V_{i}} \subset W^{\mathfrak{a}}$ be the Weyl group of $P_{V_{i}}$, i.e., $W_{V_{i}}$ is the stabilizer of the vertex $V_{i}$, and let $W_{E_{i}} \subset W^{\mathfrak{a}}$ be the Weyl group of $P_{E_{i}}$, i.e., $W_{E_{i}}$ is the stabilizer of the edge $E_{i}$. 
Lemma 1. Let $\gamma=\left(V_{0} \subset E_{0} \supset V_{1} \subset \cdots \supset V_{r} \subset E_{r} \supset V_{r+1}\right)$ be a combinatorial oneskeleton gallery. The set $\Gamma\left(t_{\gamma}, V_{0}\right)$ can be identified with sequences of Weyl group classes in $\prod_{i=0}^{r} W_{V_{i}} / W_{E_{i}}$ via the map

$$
\begin{aligned}
\left(w_{0}, \ldots, w_{r}\right) \mapsto \quad\left(V_{0} \subset w_{0}\left(E_{0}\right) \supset\right. & w_{0}\left(V_{1}\right) \subset w_{0} w_{1}\left(E_{1}\right) \supset \cdots \\
& \left.\cdots \subset w_{0} \cdots w_{r}\left(E_{r}\right) \supset w_{0} \cdots w_{r}\left(V_{r+1}\right)\right) .
\end{aligned}
$$

In particular, the set $\Gamma\left(t_{\gamma}, V_{0}\right)$ is finite.

Proof. Let $\gamma^{\prime}=\left(V_{0} \subset E_{0}^{\prime} \supset V_{1}^{\prime} \subset E_{1}^{\prime} \supset V_{2}^{\prime} \supset \ldots\right)$ be a one-skeleton gallery in $\Gamma\left(t_{\gamma}, V_{0}\right)$. Since the type of $E_{0}$ and $E_{0}^{\prime}$ are the same, the two have to be conjugate by the finite reflection group $W_{V_{0}}$ generated by all affine reflections $s_{\alpha, n}$ such that $V_{0} \subset \mathrm{H}_{\alpha, n}$. Proceeding by induction, we see that the map defined above is a bijection. Therefore

$$
\left|\Gamma\left(t_{\gamma}, V_{0}\right)\right|=\sum_{i=1}^{r}\left|W_{V_{i}} / W_{E_{i}}\right|,
$$

in particular, the set $\Gamma\left(t_{\gamma}, V_{0}\right)$ is finite.

\subsection{Young tableaux and one-skeleton galleries for classical groups of type $\mathrm{A}_{n}, \mathrm{~B}_{n}, \mathrm{C}_{n}$}

Throughout this section we use the Bourbaki enumeration of the weights and coweights. Given a partition $p=\left(p_{1}, \ldots, p_{n}\right)$, the associated Young diagram of shape $p$ consists of left justified rows of boxes with $p_{1}$ boxes in the first row, $p_{2}$ boxes in the second row, etc. We enumerate the rows from top to bottom $\left(R_{1}, \ldots\right)$ and the columns from the right to the left $\left(C_{1}, \ldots\right)$.

Young tableaux of type $A_{\mathrm{n}}$ : For a dominant coweight $\lambda=\sum_{i=1}^{n} a_{i} \omega_{i}$ set $p_{i}=a_{i}+\ldots+a_{n}$, we call $p_{\underline{\lambda}}=\left(p_{1}, \ldots, p_{n}\right)$ the associated partition. By a Young tableau $\mathcal{T}$ of shape $p_{\underline{\lambda}}$ and type $\mathrm{A}_{\mathrm{n}}$ we mean a filling of the boxes of the Young diagram of shape $p_{\underline{\lambda}}$ with positive integers such that the entries are smaller or equal to $n+1$ and the entries are strictly increasing in the columns (top to bottom). The tableau is called semistandard if in addition the entries are weakly increasing in the rows (left to right).

We use the linearly ordered alphabet $\mathfrak{N}=\{1<2<\ldots<n<\bar{n}<\ldots<\overline{2}<\overline{1}\}$ with the convention $\overline{\bar{i}}=i$.

Young tableaux of type $\mathrm{B}_{\mathrm{n}}$ : For a dominant coweight $\lambda=\sum_{i=1}^{n} a_{i} \omega_{i}$ set $p_{i}=2 a_{i}+$ $\ldots+2 a_{n-1}+a_{n}$, we call $p_{\underline{\lambda}}=\left(p_{1}, \ldots, p_{n}\right)$ the associated partition. By a Young tableau of shape $p_{\underline{\lambda}}$ and type $\mathrm{B}_{\mathrm{n}}$ we mean a filling of the boxes of the Young diagram of shape $p_{\underline{\lambda}}$ with elements of $\mathfrak{N}$ such that the entries are strictly increasing in the columns (top to bottom), and $i$ and $\bar{i}$ are never entries in the same column. Further, for each pair of columns $\left(C_{2 j-1}, C_{2 j}\right)$, $j=1, \ldots, a_{1}+\ldots+a_{n-1}$, either $C_{2 j-1}=C_{2 j}$ or the column $C_{2 j}$ is obtained from $C_{2 j-1}$ by exchanging some of the entries $k, 1 \leq k \leq \overline{1}$, in $C_{2 j-1}$ by $\bar{k}$. The tableau is called semistandard if in addition the entries are weakly increasing in the rows (left to right).

Young tableaux of type $\mathrm{C}_{\mathrm{n}}$ : For a dominant coweight $\lambda=\sum_{i=1}^{n} a_{i} \omega_{i}$ set $p_{1}=a_{1}+\sum_{j=2}^{n} 2 a_{j}$ and for $i \geq 2$ set $p_{i}=2 a_{i}+\ldots+2 a_{n}$, we call $p_{\underline{\lambda}}=\left(p_{1}, \ldots, p_{n}\right)$ the associated partition. By a Young tableau of shape $p_{\underline{\lambda}}$ and type $\mathrm{C}_{\mathrm{n}}$ we mean a filling of the boxes of the Young diagram of shape $p_{\underline{\lambda}}$ with elements of $\mathfrak{N}$, strictly increasing in the columns (top to bottom), but $i$ and $\bar{i}$ are never entries in the same column. Further, for each pair of columns $\left(C_{a_{1}+2 j-1}, C_{a_{1}+2 j}\right)$, 
$j=1, \ldots, a_{2}+\ldots+a_{n}$, either $C_{a_{1}+2 j-1}=C_{a_{1}+2 j}$ or the column $C_{a_{1}+2 j}$ is obtained from $C_{a_{1}+2 j-1}$ by exchanging for an even number of times an entry $k, 1 \leq k \leq \overline{1}$, in $C_{a_{1}+2 j-1}$ by $\bar{k}$. The tableau is called semistandard if in addition the entries are weakly increasing in the rows (left to right).

Example 5. The following tableaux are semistandard Young tableaux of shape $p_{\underline{\lambda}}$ for $\lambda=$ $\omega_{1}+\omega_{2}+\omega_{3}:$

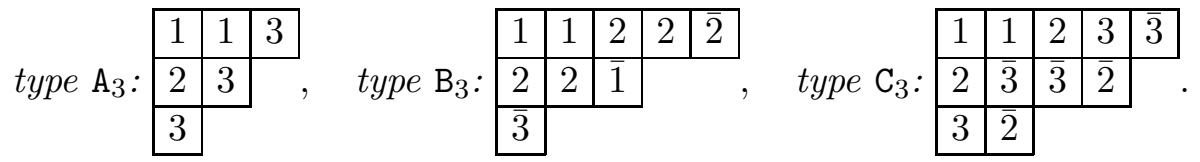

The one-skeleton gallery $\gamma_{\omega_{i}}$ :

(1) If $\omega_{i}$ is a minuscule fundamental coweight (i.e. $i$ is arbitrary for type $\mathrm{A}_{n}, i=1$ for type $\mathrm{C}_{n}, i=n$ for type $\left.\mathrm{B}_{n}\right)$, then $\gamma_{\omega_{i}}=\left(\mathfrak{o} \subset E \supset \omega_{i}\right)$, where $E$ is the closed face $\left\{t \omega_{i} \mid t \in[0,1]\right\}$. The galleries of the same type as $\gamma_{\omega_{i}}$ are the galleries $\gamma_{\sigma\left(\omega_{i}\right)}=\left(\mathfrak{o} \subset \sigma(E) \supset \sigma\left(\omega_{i}\right)\right)$, where $\sigma(E)=\left\{t \sigma\left(\omega_{i}\right) \mid t \in[0,1]\right\}$ and $\sigma \in W / W_{\omega_{i}}$. It follows that the gallery is completely determined by the weight $\sigma\left(\omega_{i}\right)$.

(2) If $\omega_{i}$ is a not a minuscule fundamental weight, then $\left\langle\omega_{i}, \beta^{\vee}\right\rangle \leq 2$ for all positive roots (because we consider only groups of classical type), and there exists at least one root such that $\left\langle\omega_{i}, \beta^{\vee}\right\rangle=2$. Hence $\gamma_{\omega_{i}}=\left(\mathfrak{o} \subset E_{1} \supset V \subset E_{2} \supset \omega_{i}\right)$, where $V=\frac{1}{2} \omega_{i}, E_{1}$ is the closed face $\left\{t \omega_{i} \mid t \in\left[0, \frac{1}{2}\right]\right\}$ and $E_{2}$ is the displaced face $E_{1}+\frac{1}{2} \omega_{i}=\left\{t \omega_{i} \mid t \in\left[\frac{1}{2}, 1\right]\right\}$. The galleries of the same type having $E_{1}$ as a first one-dimensional face are of the form $\gamma=\left(\mathfrak{o} \subset E_{1} \supset V \subset \sigma\left(E_{1}\right)+\frac{1}{2} \omega_{i} \supset \frac{\omega_{i}+\sigma\left(\omega_{i}\right)}{2}\right)$, where $\sigma$ is an element in the subgroup $W_{V}^{v}$ of $W$ generated by the simple reflection $s_{\alpha_{j}}, j \neq i$, and the reflection $s_{\beta}$, where $\beta$ is the dominant short root. An arbitrary one-skeleton gallery of the same type as $\gamma_{\omega_{i}}$ is of the form $\gamma=\left(\mathfrak{o} \subset \tau\left(E_{1}\right) \supset \frac{\tau\left(\omega_{i}\right)}{2} \subset \tau \sigma\left(E_{1}\right)+\frac{1}{2} \tau\left(\omega_{i}\right) \supset \frac{\tau\left(\omega_{i}\right)+\tau \sigma\left(\omega_{i}\right)}{2}\right)$, where $\tau \in W$ and $\sigma$ is a above. So the gallery is completely determined by the pair of weights $\tau\left(\omega_{i}\right)$ and $\tau \sigma\left(\omega_{i}\right)$,

\section{Weights, one column tableaux and two column tableaux:}

We encode the Weyl group conjugates of a fundamental coweight in a tableau consisting of one column. Then $\omega_{i}=\epsilon_{1}+\ldots+\epsilon_{i}$ for $i=1, \ldots, n$, except for type $\mathrm{B}_{n}$, in this case $\omega_{n}=\frac{1}{2}\left(\epsilon_{1}+\ldots+\epsilon_{n}\right)$. To have a uniform notation, for $1 \leq i \leq n$ we write $\epsilon_{\bar{i}}$ for $-\epsilon_{i}$. In type $\mathrm{A}_{n}$ we have $W \cdot \omega_{i}=\left\{\epsilon_{j_{1}}+\ldots+\epsilon_{j_{i}} \mid 1 \leq i_{1}<\ldots<j_{i} \leq n+1\right\}$. By writing the indices $j_{1}<\ldots<j_{i}$ as entries in a Young tableaux of shape $p_{\underline{\omega}_{i}}$ we get a bijection between the elements in the orbit and the Young tableaux of shape $p_{\omega_{i}}$ and type $A_{n}$.

In type $\mathrm{C}_{n}$ and $\mathrm{B}_{n}$ we have $W \cdot \omega_{i}=\left\{\epsilon_{j_{1}}+\ldots+\epsilon_{j_{i}} \mid 1 \leq j_{1}<\ldots<j_{i} \leq \overline{1}, \forall k, \ell: j_{k} \neq \bar{j}_{\ell}\right\}$, except for $\omega_{n}$ in type $\mathrm{B}_{n}$, in this case $W \cdot \omega_{n}=\left\{\frac{1}{2}\left(\epsilon_{j_{1}}+\ldots+\epsilon_{j_{n}}\right) \mid 1 \leq j_{1}<\ldots<j_{n} \leq\right.$ $\left.\overline{1}, \forall k, \ell: j_{k} \neq \bar{j}_{\ell}\right\}$. So by writing the indices as entries in a Young tableaux consisting of one column with $i$ boxes, this provides a bijection between the orbit $W \cdot \omega_{i}$ and the one column Young tableaux satisfying the column conditions in the definition of Young tableaux of type $\mathrm{B}_{n}$ and $\mathrm{C}_{n}$. In particular:

Lemma 2. If $\omega_{i}$ is minuscule, then this correspondence gives a bijection between the galleries of the same type as $\gamma_{\omega_{i}}$ and the set of Young tableaux of shape $p_{\underline{\omega_{i}}}$ and type $\mathrm{A}_{n}$ respectively $\mathrm{B}_{n}$ or $\mathrm{C}_{n}$.

Suppose $\omega_{i}$ is not minuscule and

$$
\gamma=\left(\mathfrak{o} \subset \tau\left(E_{1}\right) \supset V=\frac{\tau\left(\omega_{i}\right)}{2} \subset \tau \sigma\left(E_{1}\right)+\frac{1}{2} \tau\left(\omega_{i}\right) \supset \frac{\tau\left(\omega_{i}\right)+\tau \sigma\left(\omega_{i}\right)}{2}\right)
$$


is a gallery of the same type as $\gamma_{\omega_{i}}$. If $\tau=i d$, then $W_{V}^{v}$ is a subgroup of $W$ of type $\mathrm{B}_{\mathrm{i}} \times \mathrm{B}_{\mathrm{n}-\mathrm{i}}$ for type $\mathrm{B}_{n}$ and of type $\mathrm{D}_{\mathrm{i}} \times \mathrm{C}_{n-i}$ for type $\mathrm{C}_{n}$, we write $W_{V}^{v}=\left(W_{V}^{1}\right)^{v} \times\left(W_{V}^{2}\right)^{v}$ for this product decomposition. We have seen above that the possible choices for $\sigma\left(E_{1}\right)$ is in bijection with the orbit $W_{V}^{v} \cdot \omega_{i}$. The second part in the product decomposition of $W_{V}^{v}$ is in the stabilizer of $\omega_{i}$, so the possible choices for $\sigma\left(E_{1}\right)$ are in bijection with the orbit $\left(W_{V}^{1}\right)^{v} \cdot \omega_{i}$. The weights occurring in this orbit are twice the weights of the $\operatorname{Spin}_{2 i+1}$ respectively $\operatorname{Spin}_{2 i}$ representation, i.e. the weights are obtained from $\omega_{i}=\epsilon_{1}+\ldots+\epsilon_{i}$ just by a change of the signs in the $\mathrm{B}_{n}$-case and by an even number of sign changes in the $\mathrm{C}_{n}$-case. Now if $\gamma$ is an arbitrary gallery of the same type as $\gamma_{\omega_{i}}$ as in (8) , by linearity the weight $\tau \sigma\left(\omega_{i}\right)$ is obtained from the weight $\tau\left(\omega_{i}\right)$ by a change of some signs in the $B_{n}$-case respectively by an even number of sign changes in the $\mathrm{C}_{n}$-case. So attach to $\gamma$ the (two column) tableau of shape $p_{\omega_{i}}$ having as first column the one corresponding to $\tau\left(\omega_{i}\right)$ and as second the one corresponding to $\tau \sigma\left(\omega_{i}\right)$. It follows:

Lemma 3. If $\omega_{i}$ is not minuscule, then this correspondence describes a bijection between the galleries of the same type as $\gamma_{\omega_{i}}$ and the set of Young tableaux of shape $p_{\omega_{i}}$ and type $\mathrm{B}_{n}$ respectively $\mathrm{C}_{n}$.

One-skeleton galleries and tableaux:

For a dominant coweight $\lambda=\sum a_{i} \omega_{i}$ let $\gamma_{\underline{\lambda}}$ be the concatenation of the galleries $\gamma_{\omega_{j}}$ associated to the fundamental coweights:

$$
\gamma_{\underline{\lambda}}=\underbrace{\gamma_{\omega_{1}} * \cdots * \gamma_{\omega_{1}}}_{a_{1} \text {-times }} * \cdots * \underbrace{\gamma_{\omega_{n}} * \cdots * \gamma_{\omega_{n}}}_{a_{n} \text {-times }}
$$

and let $\gamma=\gamma_{1} * \cdots * \gamma_{r}, r=\sum a_{i}$, be a gallery of the same type as $\gamma_{\underline{\lambda}}$. One can associate to $\gamma$ in a natural way a tableau of shape $p_{\underline{\lambda}}$ : fix $j$ minimal such that $a_{j} \neq 0$ and let $\mathcal{T}_{1}$ be the one- respectively two column tableau of shape $p_{\omega_{j}}$ associated to $\gamma_{1}$. Suppose we have already defined $\mathcal{T}_{k}, 1 \leq k \leq r$. If $r=k$, then set $\mathcal{T}_{\gamma}=\mathcal{T}_{k}$. If $k<r$, let $\ell$ be such that $\gamma_{k+1}$ is of shape $\omega_{\ell}$ and let $\mathcal{T}_{k+1}$ be the tableau obtained from $\mathcal{T}_{k}$ by adding to the left the one column (if $\omega_{\ell}$ is minuscule) respectively the two column tableau (if $\omega_{\ell}$ is not minuscule) corresponding to $\gamma_{k+1}$. The construction above implies:

Proposition 1. The correspondence $\gamma \leftrightarrow \mathcal{T}_{\gamma}$ describes a bijection between the set of galleries of the same type as $\gamma_{\underline{\lambda}}$ and the set of Young tableaux of shape $p_{\underline{\lambda}}$ and type $\mathrm{A}_{n}$ respectively $\mathrm{B}_{n}$ or $\mathrm{C}_{n}$.

\subsection{Varieties of galleries and Bott-Samelson varieties}

Fix a combinatorial one-skeleton gallery $\gamma=\left(V_{0}=\mathfrak{o} \subset E_{0} \supset V_{1} \subset \cdots \supset V_{r} \subset E_{r} \supset V_{r+1}\right)$, we can associate to the gallery a sequence of parahoric subgroups:

$$
G(\mathcal{O}) \supset P_{E_{0}^{f}} \subset P_{V_{1}^{f}} \supset \ldots \subset P_{V_{r}^{f}} \supset P_{E_{r}^{f}} \subset P_{V_{r+1}^{f}} .
$$

We use now this correspondence to identify one-skeleton galleries with points in (generalized) Bott-Samelson varieties.

Definition 8. The variety $\Sigma\left(t_{\gamma}\right)$ of galleries of type $t_{\gamma}$ starting in $V_{0}=\mathfrak{o}$ is the closed subvariety of

$$
G(\mathcal{K}) / G(\mathcal{O}) \times G(\mathcal{K}) / P_{E_{0}^{f}} \times \ldots \times G(\mathcal{K}) / P_{E_{r}^{f}} \times G(\mathcal{K}) / P_{V_{r+1}^{f}}
$$


given by all sequences of parahoric subgroups of shape

$$
G(\mathcal{O}) \supset Q_{0} \subset R_{1} \supset Q_{1} \subset \cdots \supset Q_{r} \subset R_{r+1},
$$

where $R_{i}$ is conjugate to $P_{V_{i}^{f}}$ for $i=1, \ldots, r+1$ and $Q_{i}$ is conjugate to $P_{E_{i}^{f}}$ for $i=0, \ldots, r$.

The action of the group $G(\mathcal{K})$ on $\mathcal{J}^{\mathfrak{a}}$ naturally extends to an action of $G(\mathcal{K})$ on the set of galleries. The action of $G(\mathcal{K})$ is type preserving, the variety of galleries of fixed type $\Sigma\left(t_{\gamma}\right)$ starting in $V_{0}$ is stable under the action of $G(\mathcal{O})$. Because of the bijection of parahoric subgroups with faces of $\mathcal{J}^{\mathfrak{a}}$, the set of all points of the variety $\Sigma\left(t_{\gamma}\right)$ is in bijection with the one-skeleton galleries in $\mathcal{J}^{\mathfrak{a}}$

$$
g=\left(V_{0}=\mathfrak{o} \subset E_{0}^{\prime} \supset V_{1}^{\prime} \subset E_{1}^{\prime} \supset \cdots \subset E_{r}^{\prime} \supset V_{r+1}^{\prime}\right)
$$

having type $t_{\gamma}$. The combinatorial galleries correspond to sequences of subgroups conjugated to the $P_{E_{i}^{f}}$ 's and $P_{V_{i}^{f}}$ 's by elements in $W^{\mathfrak{a}}$, these are precisely the $T$-fixed points in $\Sigma\left(t_{\gamma}\right)$. Given a sequence of parahoric subgroups

$$
G(\mathcal{O}) \supset P_{E_{0}^{f}} \subset P_{V_{1}^{f}} \supset P_{E_{0}^{f}} \subset \cdots \supset P_{E_{r}^{f}} \subset P_{V_{r+1}^{f}},
$$

one defines the fibred product

$$
G(\mathcal{O}) \times P_{E_{0}^{f}} P_{V_{1}^{f}} \times P_{E_{1}^{f}} \cdots \times P_{E_{r-1}^{f}} P_{V_{r}^{f}} / P_{E_{r}^{f}}
$$

as the quotient of $P_{V_{0}^{f}} \times P_{V_{1}^{f}} \times \cdots \times P_{V_{r}^{f}}$ by $P_{E_{0}^{f}} \times P_{E_{1}^{f}} \times \cdots \times P_{E_{r}^{f}}$ given by the action :

$$
\left(p_{0}, p_{1}, \ldots, p_{r}\right) \cdot\left(q_{0}, q_{1}, \ldots, q_{r}\right)=\left(q_{0} p_{0}, p_{0}^{-1} q_{1} p_{1}, \ldots, p_{r-1}^{-1} q_{r} p_{r}\right)
$$

This fibred product is a smooth projective complex variety. Its points are denoted by $\left[g_{0}, \ldots, g_{r}\right]$. The following proposition is proved in [7] in the case of varieties of galleries in the spherical building associated to a semi-simple group. The proof extends naturally to our setting.

Proposition 2. As a variety, $\Sigma\left(t_{\gamma}\right)$ is isomorphic to the fibred product via the map

$$
\begin{aligned}
& {\left[g_{0}, \ldots, g_{r}\right] \mapsto \quad\left(P_{V_{0}} \supset g_{0} P_{E_{0}} g_{0}^{-1} \subset g_{0} P_{V_{1}} g_{0}^{-1} \supset g_{0} g_{1} P_{E_{1}} g_{1}^{-1} g_{0}^{-1} \subset \cdots\right.} \\
& \cdots\left.\subset g_{0} \cdots g_{r} P_{V_{r+1}} g_{r}^{-1} \cdots g_{0}^{-1}\right) .
\end{aligned}
$$

Given a dominant coweight $\lambda$, let $\gamma_{\lambda}=\left(\mathfrak{o} \subset E_{0} \supset \ldots \subset E_{r} \supset \lambda\right)$ be a corresponding one-skeleton gallery as in Example 4. In this case, the variety of galleries of type $t_{\gamma_{\lambda}}$ starting in $\mathfrak{o}$ is called the Bott-Samelson variety associated to the gallery $\gamma_{\lambda}$ and is denoted by:

$$
\Sigma\left(\gamma_{\lambda}\right):=G(\mathcal{O}) \times{ }_{E_{0}^{f}} P_{V_{1}^{f}} \times P_{E_{1}^{f}} \ldots P_{V_{r-1}^{f}} \times P_{E_{r-1}^{f}} P_{V_{r}^{f}} / P_{E_{r}^{f}}
$$

The set of all combinatorial galleries in the Bott-Samelson variety is denoted by $\Gamma\left(\gamma_{\lambda}\right)$. For instance, the gallery $\gamma_{\lambda}$ corresponds to $\left[1, w_{1}, \ldots, w_{r}\right]$, where $w_{j}$ is the minimal representative of the longest element in $W_{V_{j}^{f}} / W_{E_{j}^{f}}$.

Example 6. If $\omega$ is a minuscule fundamental coweight, then $\gamma_{\omega}=\left(\mathfrak{o} \subset E_{0} \supset \omega\right)$ and $\Sigma\left(\gamma_{\omega}\right)=$ $G(\mathcal{O}) / P_{E_{0}}$ is just a homogeneous space, isomorphic to the orbit $G(\mathcal{O}) \cdot \omega=\overline{G(\mathcal{O}) \cdot \omega}=X_{\omega}$. 
In general, the connection between $X_{\lambda}$ and $\Sigma\left(\gamma_{\lambda}\right)$ is given by the following proposition:

Proposition 3. As in Definition [6, denote by $\lambda^{f}$ also the point in $\mathcal{G}=G(\mathcal{K}) / G(\mathcal{O})$ corresponding to the vertex of the fundamental alcove of the same type as $\lambda$. The canonical product map

$$
\begin{aligned}
\pi: \Sigma\left(\gamma_{\lambda}\right):=G(\mathcal{O}) \times{ }_{P_{E_{0}}} P_{V_{1}} \times{ }_{P_{E_{1}^{f}}} \ldots P_{V_{r}^{f}} / P_{E_{r}^{f}} & \rightarrow \mathcal{G} \\
{\left[g_{0}, g_{1}, \ldots, g_{r}\right] } & \mapsto g_{0} g_{1} \cdots g_{r} \lambda^{f}
\end{aligned}
$$

has as image the Schubert variety $X_{\lambda}$. The induced map $\pi: \Sigma\left(\gamma_{\lambda}\right) \rightarrow X_{\lambda}$ defines a desingularization of the variety $X_{\lambda}$.

The proof of this proposition is similar to the proof in the classical case and is based on the fact that the gallery $\gamma_{\lambda}$ is minimal. The notion of minimality in our context is defined and discussed in Section 5 .

\subsection{Cells}

Let $\eta: \mathbb{C}^{*} \rightarrow T$ be a generic anti-dominant coweight. Then the set of $\eta$-fixed-points in $\Sigma\left(\gamma_{\lambda}\right)$ is finite and is in bijection with the set of all combinatorial galleries of the same type as $\gamma_{\lambda}$. For such a fixed $\gamma$ denote by $C_{\gamma}$ the corresponding Białynicki-Birula cell, i.e. the set of points such that $\lim _{t \rightarrow 0} \eta(t) \cdot x=\gamma$.

For a face $F$ in $\mathcal{J}^{\mathfrak{a}}, \lim _{t \rightarrow 0} \eta(t) . F=r_{-\infty}(F)$, and for a face $F$ in $\mathbb{A}, r_{-\infty}^{-1}(F)=U^{-}(\mathcal{K}) . F$. Therefore, we want to determine as precisely as possible the group $\operatorname{Stab}_{-}(F)=\operatorname{Stab}_{U^{-}(\mathcal{K})}(F)$ and the set $\mathrm{Stab}_{-}(V) / \operatorname{Stab}_{-}(F)$ when $F$ and $V$ are faces of the Coxeter complex such that $V \subset F$.

Bruhat and Tits (see (7.1.1) in [5]) associate to a face $F$ of the Coxeter complex the function $f_{F}: \alpha \mapsto \inf _{k \in \mathbb{Z}}\{\alpha(F)+k \geq 0\}$. If $\alpha \in \Phi$, then $f_{F}(\alpha)$ is the smallest integer $n$ such that $F$ lies in the closed half-space $H_{\alpha, n}^{+}$. The function $f_{F}$ is convex and positively homogeneous of degree 1 ; in particular, $f_{F}(i \alpha+j \beta) \leqslant i f_{F}(\alpha)+j f_{F}(\beta)$ for all roots $\alpha, \beta \in \Phi$ and all positive integers $i, j$.

When $F$ and $V$ are two faces of $\mathbb{A}$ such that $V \subset F$, then we denote by $\Phi_{-}^{\mathfrak{a}}(V, F)$ the set of all affine roots $\beta \in \Phi_{-} \times \mathbb{Z}$ such that $V \subset \mathrm{H}_{\beta}$ and $F \not \subset H_{\beta}^{+}$; in other words, $(\alpha, n) \in \Phi_{-}^{\mathfrak{a}}(V, F)$ if and only if $\alpha \in \Phi_{-}, n=f_{V}(\alpha)$ and $n+1=f_{F}(\alpha)$. We denote by $\operatorname{Stab}_{-}(V, F)$ the subgroup of $U^{-}(\mathcal{K})$ generated by the elements of the form $x_{\beta}(a)$ with $\beta \in \Phi_{-}^{\mathfrak{a}}(V, F)$ and $a \in \mathbb{C}$. We plot an example to help the understanding of all these definitions. In the following picture, $\alpha$ is a positive root.

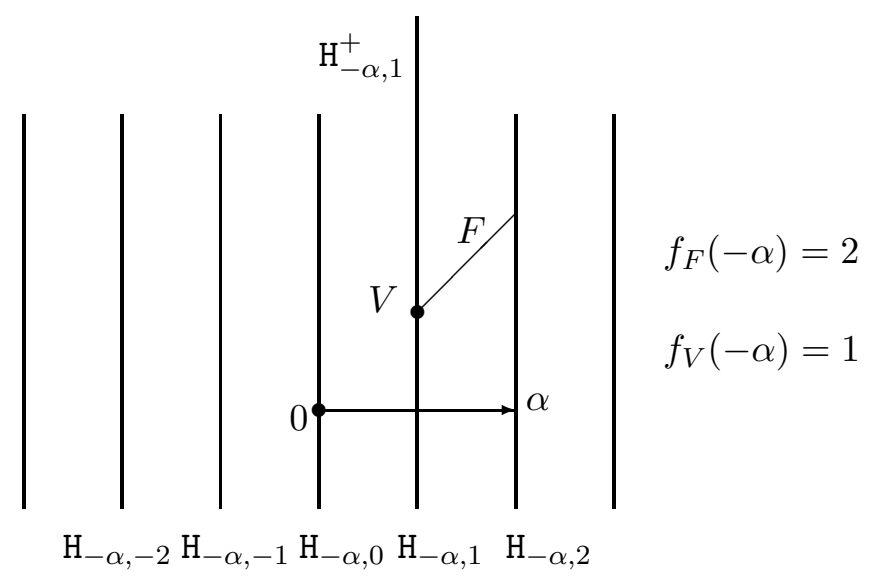


The following proposition is proved in [1, Proposition 19.

Proposition 4. 1. The stabilizer $\mathrm{Stab}_{-}(F)$ of a face $F$ of the Coxeter complex is generated by the elements $x_{\alpha}(p)$, where $\alpha \in \Phi_{-}$and $p \in \mathcal{K}$ satisfy $\operatorname{val}(p) \geqslant f_{F}(\alpha)$.

2. Let $F$ and $V$ be two faces of the Coxeter complex such that $V \subset F$. Then Stab_ $(V, F)$ is a set of representatives for the right cosets of $\mathrm{Stab}_{-}(F)$ in $\mathrm{Stab}_{-}(V)$. For any total order on the set $\Phi_{-}^{\mathfrak{a}}(V, F)$, the map

$$
\left(a_{\beta}\right)_{\beta \in \Phi_{-}^{\mathfrak{a}}(V, F)} \mapsto \prod_{\beta \in \Phi_{-}^{\mathfrak{a}}(V, F)} x_{\beta}\left(a_{\beta}\right)
$$

is a bijection from $\mathbb{C}_{-}^{\Phi_{-}^{\mathfrak{a}}(V, F)}$ onto $\mathrm{Stab}_{-}(V, F)$, where $\mathbb{C}^{\Phi_{-}^{\mathfrak{a}}(V, F)}$ is the set of all mappings from $\Phi_{-}^{\mathfrak{a}}(V, F)$ to $\mathbb{C}$.

In $\mathcal{J}_{V}^{\mathfrak{a}}, F_{V}$ corresponds to a spherical face of dimension one given by an element $\overline{w_{F}} \in$ $W_{V} / W_{F}$ such that $F=\overline{w_{F}} \phi_{F}^{-}$, where $\phi_{F}^{-}$is the face having the same type as $F_{V}$ contained in $C_{V}^{-}$. Let $D=\operatorname{proj}_{F}\left(C_{V}^{-}\right)$be the closest chamber to $C_{V}^{-}$containing $F_{V}$, then $\overline{w_{F}}=\overline{w\left(C_{V}^{-}, D\right)}$.

Proposition 5. The walls $\mathrm{H}_{\beta}, \beta \in \Phi_{-}^{\mathfrak{a}}(V, F)$, viewed as walls in $\mathbb{A}_{V}$, are the walls crossed by any minimal gallery of chambers between $C_{V}^{-}$and $D$.

Proof. By definition, $\Phi_{-}^{\mathfrak{a}}(V, F)=\left\{\beta \in \Phi_{-} \times \mathbb{Z} \mid V \subset \mathrm{H}_{\beta}, F \not \subset H_{\beta}^{+}\right\}$. So, for any $\beta$ in this set, the wall $\mathrm{H}_{\beta}$ separates $C_{V}^{-}$from $F_{V}$. Moreover, $F \not \subset H_{\beta}^{+}$implies that it separates also $C_{V}^{-}$ from $D$. Hence, $\mathrm{H}_{\beta}$ is crossed by any minimal gallery of chambers between $C_{V}^{-}$and $D$.

Therefore, $\mathrm{Stab}_{-}(V, F)$ can be identified with $U_{V}^{-}\left(\overline{w_{F}}\right)$, where the latter is defined as $B_{V}^{-} \overline{w_{F}} P_{F}^{-} / P_{F}^{-}=U_{V}^{-}\left(\overline{w_{F}}\right) \overline{w_{F}} P_{F}^{-} / P_{F}^{-}$. Let

$$
\delta=\left[\delta_{0}, \delta_{1}, \ldots, \delta_{r}\right]=\left(0=V_{0} \subset E_{0} \supset V_{1} \subset \cdots \supset V_{r} \subset E_{r} \supset V_{r+1}\right) \in \Gamma\left(\gamma_{\lambda}\right)
$$

and set

$$
\operatorname{Stab}_{-}(\delta)=\operatorname{Stab}_{-}\left(V_{0}, E_{0}\right) \times \operatorname{Stab}_{-}\left(V_{1}, E_{1}\right) \times \cdots \times \operatorname{Stab}_{-}\left(V_{r}, E_{r}\right) .
$$

Proposition 6. The map

$$
\begin{aligned}
& f:\left(v_{0}, v_{1}, \ldots, v_{r}\right) \mapsto \\
& {\left[v_{0} \overline{\delta_{0}}, \overline{\delta_{0}}{ }^{-1} v_{1} \overline{\delta_{0} \delta_{1}},{\overline{\delta_{0} \delta_{1}}}^{-1} v_{2} \overline{\delta_{0} \delta_{1} \delta_{2}}, \ldots, \overline{\delta_{0} \cdots \delta_{r-1}}-1 v_{r} \overline{\delta_{0} \cdots \delta_{r}}\right] }
\end{aligned}
$$

from Stab_ $(\delta)$ to $\Sigma\left(\gamma_{\lambda}\right)$ is injective and its image is $C_{\delta}$ (here $\bar{x}$ means that we take a coset representative of $x$ in $G(\mathcal{K}))$. Therefore, $C_{\delta}$ is isomorphic to $\mathbb{C}^{\Phi_{-}^{a}\left(V_{0}, E_{0}\right)} \times \cdots \times \mathbb{C}^{\Phi_{-}^{a}\left(V_{r}, E_{r}\right)}$.

Proof. The proof is similar to the one of Proposition 22 in [1, we give it for the comfort of the reader. Set $\widetilde{\mathrm{Stab}_{-}}(\delta)=$

$$
\mathrm{Stab}_{-}\left(V_{0}\right) \underset{\mathrm{Stab}_{-}\left(E_{0}\right)}{\times} \mathrm{Stab}_{-}\left(V_{1}\right) \underset{\mathrm{Stab}_{-}\left(E_{1}\right)}{\times} \cdots \underset{\mathrm{Stab}_{-}\left(E_{r-1}\right)}{\times} \mathrm{Stab}_{-}\left(V_{r}\right) / \mathrm{Stab}_{-}\left(E_{r}\right) .
$$


Using the inclusions

$$
\begin{array}{ll}
\operatorname{Stab}_{-}\left(E_{j}\right) \subseteq \overline{\delta_{0} \cdots \delta_{j}} P_{E_{j}^{f}}{\overline{\delta_{0} \cdots \delta_{j}}}^{-1} & (\text { for } 0 \leqslant j \leqslant r), \\
\operatorname{Stab}_{-}\left(V_{0}\right) \subseteq G(\mathcal{O}) \bar{\delta}_{0}^{-1}, & \\
\operatorname{Stab}_{-}\left(V_{j}\right) \subseteq \overline{\delta_{0} \cdots \delta_{j-1}} P_{V_{j}^{f}}{\overline{\delta_{0} \cdots \delta_{j}}}^{-1} & (\text { for } 1 \leqslant j \leqslant r),
\end{array}
$$

standard arguments imply that the map

$$
\begin{aligned}
& f:\left[v_{0}, v_{1}, \ldots, v_{r}\right] \mapsto \\
& {\left[v_{0} \overline{\delta_{0}}, \overline{\delta_{0}}{ }^{-1} v_{1} \overline{\delta_{0} \delta_{1}}, \overline{\delta_{0} \delta_{1}}-1 v_{2} \overline{\delta_{0} \delta_{1} \delta_{2}}, \ldots, \overline{\delta_{0} \cdots \delta_{r-1}}-1 v_{r} \overline{\delta_{0} \cdots \delta_{r}}\right] }
\end{aligned}
$$

from $\widetilde{\operatorname{Stab}_{-}(\delta)}$ to $\hat{\Sigma}\left(\gamma_{\lambda}\right)$ is well-defined.

The proof of Proposition 6 in [9] says that an element $d=\left[g_{0}, g_{1}, \ldots, g_{r}\right]$ in the BottSamelson variety belongs to the cell $C_{\delta}$ if and only if there exists $u_{0}, u_{1}, \ldots, u_{r} \in U^{-}(\mathcal{K})$ such that

$$
g_{0} g_{1} \cdots g_{j} E_{j}^{f}=u_{j} E_{j} \quad \text { and } \quad u_{j-1} V_{j}=u_{j} V_{j}
$$

for each $j$. Setting $v_{0}=u_{0}$ and $v_{j}=u_{j-1}^{-1} u_{j}$ for $1 \leqslant j \leqslant r$, the conditions above can be rewritten

$$
g_{0} g_{1} \cdots g_{j} P_{E_{j}^{f}}=v_{0} v_{1} \cdots v_{j} \overline{\delta_{0} \delta_{1} \cdots \delta_{j}} P_{E_{j}^{f}} \quad \text { and } \quad v_{j} \in \operatorname{Stab}_{-}\left(V_{j}\right),
$$

which shows that $f\left(\left[v_{0}, v_{1}, \ldots, v_{r}\right]\right)=d$. Therefore the image of $f$ contains the cell $C_{\delta}$. The reverse inclusion can be established similarly.

The map $f$ is injective. Indeed suppose that two elements $v=\left[v_{0}, v_{1}, \ldots, v_{r}\right]$ and $v^{\prime}=$ $\left[v_{0}^{\prime}, v_{1}^{\prime}, \ldots, v_{r}^{\prime}\right]$ in $\left.\widetilde{\operatorname{Stab}_{-}(} \delta\right)$ have the same image. Then

$$
v_{0} v_{1} \cdots v_{j} \overline{\delta_{0} \delta_{1} \cdots \delta_{j}} P_{E_{j}^{f}}=v_{0}^{\prime} v_{1}^{\prime} \cdots v_{j}^{\prime} \overline{\delta_{0} \delta_{1} \cdots \delta_{j}} P_{E_{j}^{f}}
$$

for each $j \in\{0, \ldots, r\}$. This means geometrically that

$$
v_{0} v_{1} \cdots v_{j} \overline{\delta_{0} \delta_{1} \cdots \delta_{j}} E_{j}^{f}=v_{0}^{\prime} v_{1}^{\prime} \cdots v_{j}^{\prime} \overline{\delta_{0} \delta_{1} \cdots \delta_{j}} E_{j}^{f}
$$

in other words, $v_{0} v_{1} \cdots v_{j}$ and $v_{0}^{\prime} v_{1}^{\prime} \cdots v_{j}^{\prime}$ are equal in $U^{-}(\mathcal{K}) / \operatorname{Stab}_{-}\left(E_{j}\right)$. Since this holds for each $j$, the two elements $v$ and $v^{\prime}$ are equal in $\widetilde{\operatorname{Stab}_{-}(\delta)}$.

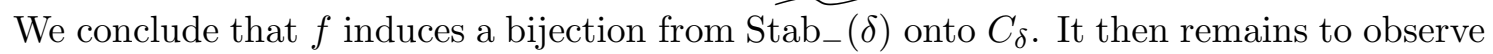
that the map $\left(v_{0}, v_{1}, \ldots, v_{r}\right) \mapsto\left[v_{0}, v_{1}, \ldots, v_{r}\right]$ from $\operatorname{Stab}_{-}(\delta)$ to $\widetilde{\operatorname{Stab}_{-}(\delta)}$ is bijective. This follows from Proposition 4 part 2 , indeed for each $\left[a_{0}, a_{1}, \ldots, a_{r}\right] \in \widehat{\operatorname{Stab}_{-}(\delta)}$, the element $\left(v_{0}, v_{1}, \ldots, v_{r}\right) \in \mathrm{Stab}_{-}(\delta)$ such that $\left[v_{0}, v_{1}, \ldots, v_{r}\right]=\left[a_{0}, a_{1}, \ldots, a_{r}\right]$ is uniquely determined by the condition that for all $j \in\{0,1, \ldots, r\}$,

$$
v_{j} \in\left(\left(v_{0} \cdots v_{j-1}\right)^{-1}\left(a_{0} \cdots a_{j}\right) \operatorname{Stab}_{-}\left(E_{j}\right)\right) \cap \operatorname{Stab}_{-}\left(V_{j}, E_{j}\right) .
$$




\section{Minimal one-skeleton galleries}

To study the intersection $Z_{\lambda, \mu}:=G(\mathcal{O}) \cdot \lambda \cap U^{-}(\mathcal{K}) \cdot \mu$ (see (2) ) using the language of galleries, we need to characterize which galleries in $\Sigma\left(\gamma_{\lambda}\right)$ map onto the dense orbit $G(\mathcal{O}) \cdot \lambda$ in $X_{\lambda}$ (see Corollary 1). This will be done by introducing the notion of minimal galleries. These galleries replace the minimal galleries of alcoves used in [9].

\subsection{Minimality relative to an equivalence class of sectors}

A sector $\mathfrak{s}$ in the affine building is a sector in some apartment. Two sectors are called equivalent if the intersection of the two is again a sector. Recall that for two given sectors $\mathfrak{s}_{1}, \mathfrak{s}_{2}$, there exists an apartment $A$ and subsectors $\mathfrak{s}_{1}^{\prime} \subset \mathfrak{s}_{1}, \mathfrak{s}_{2}^{\prime} \subset \mathfrak{s}_{2}$ such that $\mathfrak{s}_{1}^{\prime}, \mathfrak{s}_{2}^{\prime} \subset A$. The set of equivalence classes of sectors is in bijection with the set of Weyl chambers in $\mathbb{A}$. Given a sector $\mathfrak{s}$, we denote such an equivalence class by $\mathfrak{s}$.

Definition 9. A one-skeleton gallery

$$
\gamma=\left(V_{0} \subset E_{0} \supset V_{1} \subset E_{1} \supset \cdots \supset V_{r} \subset E_{r} \supset V_{r+1}\right)
$$

is called minimal if there exists an equivalence class of sectors $\underline{\mathfrak{s}}_{\gamma}$ and representatives $\mathfrak{s}_{0}, \ldots, \mathfrak{s}_{r} \in$ $\underline{\mathfrak{s}}_{\gamma}$ such that for all $i=0, \ldots, r: V_{i}$ is the vertex for the sector $\mathfrak{s}_{i}$ and $V_{i} \subset E_{i} \subset \mathfrak{s}_{i}$. The class $\underline{\mathfrak{s}}_{\gamma}$ is not necessarily uniquely determined by $\gamma$.

The sequence $\underline{\mathfrak{s}}(\gamma)=\left(\mathfrak{s}_{0}, \ldots, \mathfrak{s}_{r}\right)$ is called a chain of sectors associated to $\gamma$.

Example 7. The galleries described in Examples 1,2, 3 and 4] are minimal galleries such that $\underline{\mathfrak{s}}_{\gamma}=\underline{C}^{+}$.

Remark 1. 1) With a little extra effort, one can see that this definition is an "instance" of Definition 5.24 of [7], where Contou-Carrère defines generalized minimal galleries in a Coxeter complex.

2) Thinking in geometric terms one might be inclined to demand that "minimality" should be a local property, i.e. to be verified at each vertex of the gallery. This is not sufficient, see below. Propositions 7 and 8 show that the more rigid definition above is the right definition for our purpose.

Example 8. Consider the apartment of type $A_{2}$, we use the notation $\gamma_{\omega_{1}}, \gamma_{\omega_{2}}$ as in Example 2 for the fundamental weights. For an element $w$ of the Weyl group set $\gamma_{w\left(\omega_{i}\right)}:=w\left(\gamma_{\omega_{i}}\right), i=1,2$.

The galleries $\gamma_{1}=\gamma_{s_{1}\left(\omega_{1}\right)} * \gamma_{s_{1} s_{2}\left(\omega_{2}\right)}$ and $\gamma_{2}=\gamma_{s_{1} s_{2}\left(\omega_{2}\right)} * \gamma_{s_{2} s_{1}\left(\omega_{1}\right)}$ are minimal with $\underline{\mathfrak{s}}_{\gamma_{1}}=$ $\underline{s_{1} s_{2}\left(C^{+}\right)}$and $\underline{\mathfrak{s}_{2}}=\underline{s_{1} s_{2} s_{1}\left(C^{+}\right)}$. But the gallery $\gamma:=\gamma_{s_{1}\left(\omega_{1}\right)} * \gamma_{s_{1} s_{2}\left(\omega_{2}\right)} * \gamma_{s_{2} s_{1}\left(\omega_{1}\right)}$ is not minimal in the sense above.

The natural action of $G(\mathcal{K})$ on $\mathcal{J}^{\mathfrak{a}}$ induces a natural action on one-skeleton galleries: Let $\gamma$ be a one-skeleton gallery and $\mathbf{g} \in G(\mathcal{K})$, then we set

$$
\text { g. } \gamma=\left(\text { g. } V_{0} \subset \text { g. } E_{0} \supset \text { g. } V_{1} \subset \text { g. } E_{1} \supset \cdots \supset \text { g. } V_{r} \subset \text { g. } E_{r} \supset \text { g. } V_{r+1}\right)
$$

It follows immediately that the property of being minimal is preserved by the action. Let $\mathfrak{o}$ be the origin in $\mathbb{A}$.

Proposition 7. Let $\gamma$ be a minimal one-skeleton gallery in the building $\mathcal{J}^{\mathfrak{a}}$ starting in $V_{0}=\mathfrak{o}$ and let $\underline{\mathfrak{s}}(\gamma)=\left(\mathfrak{s}_{0}, \ldots, \mathfrak{s}_{r}\right)$ be an associated chain of sectors. 
a) $\gamma$ is contained in $\mathfrak{s}_{0}$.

b) For all $i=0, \ldots, r+1:\left(V_{i} \subset E_{i} \supset V_{i+1} \subset \cdots \supset V_{r+1}\right) \subset \mathfrak{s}_{0}\left(V_{i}\right)$. In particular, one may choose as associated chain of sectors $\mathfrak{s}(\gamma)=\left(\mathfrak{s}_{0}, \mathfrak{s}_{0}\left(V_{1}\right), \ldots, \mathfrak{s}_{0}\left(V_{r}\right)\right)$.

c) There exists a unique gallery $\gamma^{\prime}$ in the orbit $G(\mathcal{O}) \cdot \gamma$ such that $\gamma^{\prime}$ is contained in the dominant Weyl chamber $C^{+}$in $\mathbb{A}$ and the chain of sectors associated to $\gamma$ can be chosen to be all in the class of $\mathrm{C}^{+}$.

Proof. The sectors $\mathfrak{s}_{i}, \mathfrak{s}_{i+1}, 0 \leq i \leq r-1$, are in the same equivalence class, so there exists a subsector $\mathfrak{s}_{i}^{\prime}$ contained in both sectors. A sector is the closure of the convex hull of its vertex and any subsector, and hence $\mathfrak{s}_{i}$ is the closure of the convex hull of $V_{i}$ and $\mathfrak{s}_{i}^{\prime}$, and $\mathfrak{s}_{i+1}$ is the closure of the convex hull of $V_{i+1}$ and $\mathfrak{s}_{i}^{\prime}$. Since $\mathfrak{s}_{i} \supset E_{i} \supset V_{i+1}$ it follows that $\mathfrak{s}_{i+1}$ is a subsector of $\mathfrak{s}_{i}$, in fact, $\mathfrak{s}_{i+1}=\mathfrak{s}_{i}\left(V_{i+1}\right)$. By induction we conclude:

$$
\mathfrak{s}_{0} \subset \mathfrak{s}_{0}\left(V_{1}\right)=\mathfrak{s}_{1} \subset \mathfrak{s}_{0}\left(V_{2}\right)=\mathfrak{s}_{1}\left(V_{2}\right)=\mathfrak{s}_{2} \subset \ldots \subset \mathfrak{s}_{0}\left(V_{r}\right)=\ldots=\mathfrak{s}_{r}
$$

Now $E_{j} \subset \mathfrak{s}_{j}$ for all $j=0, \ldots, r$, so $\left(V_{i} \subset E_{i} \supset V_{i+1} \subset \cdots \supset V_{r+1}\right) \subset \mathfrak{s}_{0}\left(V_{i}\right)$, which finishes the proof of $a$ ) and $b$ ).

Since $G(\mathcal{O})$ acts transitively on the set of sectors having $\mathfrak{o}$ as vertex, there exists $\mathbf{g} \in G(\mathcal{O})$ such that $\mathbf{g} \cdot \mathfrak{s}_{0}=C^{+}$. It follows: $\gamma^{\prime}=\mathbf{g} \cdot \gamma$ is completely contained in $C^{+}$. It remains to prove the uniqueness.

Suppose now $\mathbf{g}^{\prime} \cdot \gamma=\left(V_{0}^{\prime} \subset E_{0}^{\prime} \ldots\right)$ and $\mathbf{g}^{\prime \prime} \cdot \gamma=\left(V_{0}^{\prime \prime} \subset E_{0}^{\prime \prime} \ldots\right)$ are contained in the dominant Weyl chamber and hence in $\mathbb{A}$. The action of $G(\mathcal{O})$ preserves types, so both galleries have the same gallery of types. Obviously we have $V_{0}^{\prime}=V_{0}^{\prime \prime}=\mathfrak{o}$ and $E_{0}^{\prime}=E_{0}^{\prime \prime}$ since both are faces of the same type of the fundamental alcove. It follows: $V_{1}^{\prime}=V_{1}^{\prime \prime}$. Since $\mathbf{g}^{\prime} \cdot \mathfrak{s}_{1}=\mathbf{g}^{\prime} \cdot\left(\mathfrak{s}_{0}\left(V_{1}\right)\right)=$ $C^{+}\left(V_{1}^{\prime}\right)=C^{+}\left(V_{1}^{\prime \prime}\right)=\mathbf{g}^{\prime \prime} \cdot\left(\mathfrak{s}_{0}\left(V_{1}\right)\right)=\mathbf{g}^{\prime \prime} \cdot \mathfrak{s}_{1}, E_{1}^{\prime} \supset V_{1}^{\prime}$ and $E_{1}^{\prime \prime} \supset V_{1}^{\prime}$ are faces of the same type of the same sector $C^{+}\left(V_{1}^{\prime}\right)$, so necessarily $E_{1}^{\prime}=E_{1}^{\prime \prime}$. Repeating the argument shows $\gamma=\gamma^{\prime}$.

Remark 2. In part $b$ ) above one can replace $\mathfrak{s}_{0}$ by $-\mathfrak{s}_{0}$ (see (4) for the notation), but one has to replace the "tail" of the gallery by the "head": For all $i=0, \ldots, r+1:\left(V_{0} \subset E_{0} \supset\right.$ $\left.V_{1} \subset \cdots \supset V_{i}\right) \subset-\mathfrak{s}_{0}\left(V_{i}\right)$.

\subsection{Orbits}

The following proposition gives us a precise dictionary between the language of minimal oneskeleton galleries and orbits of $G(\mathcal{O})$ in the affine Grassmannian $G(\mathcal{K}) / G(\mathcal{O})$.

Proposition 8. Let $\gamma$ be a minimal one-skeleton gallery in $\mathcal{J}^{\mathfrak{a}}$ starting in $\mathfrak{o}$ and ending in $\lambda=V_{r+1}$ in the dominant Weyl chamber $C^{+}$in $\mathbb{A}$. The target $\lambda=V_{r+1}$ is a special point and hence is a coweight, by abuse of notation we also write $\lambda$ for the corresponding point in $G(\mathcal{K}) / G(\mathcal{O})$. The following natural map between the $G(\mathcal{O})$-orbit of the gallery $\gamma$ and the $G(\mathcal{O})$-orbit of $\lambda$ in $\mathcal{G}$ is bijective:

$$
G(\mathcal{O}) \cdot \gamma \longrightarrow G(\mathcal{O}) \cdot \lambda \subset G(\mathcal{K}) / G(\mathcal{O}), \quad \text { g. } \gamma \mapsto \text { g. } \lambda .
$$

Proof. The map $\pi$ defined in Proposition 3 is $G(\mathcal{O})$-equivariant and, as a desingularization of $X(\lambda)$, it must be an isomorphism over an open subset of $X(\lambda)=\overline{G(\mathcal{O}) \cdot \lambda}$. So it restricts to a bijection $G(\mathcal{O}) \cdot \gamma \simeq G(\mathcal{O}) \cdot \lambda$.

Summarizing we have proved: 
Corollary 1. 1. Let $\gamma$ be a minimal one-skeleton gallery starting in $\mathfrak{o}$, then the $G(\mathcal{O})$-orbit of $\gamma$ contains a unique element completely contained in the dominant Weyl chamber.

2. Let $\gamma, \gamma^{\prime}$ be two minimal one-skeleton galleries starting in $\mathfrak{o}$. The two galleries are conjugate under the action of $G(\mathcal{O})$ if and only if they have the same galleries of types.

3. Let $\gamma=\left(V_{0}=\mathfrak{o} \subset E_{0} \supset V_{1} \subset E_{1} \supset \cdots \supset V_{r} \subset E_{r} \supset V_{r+1}\right)$ be a minimal one-skeleton gallery contained in the dominant Weyl chamber and let $\lambda=V_{r+1} \in X^{+}$be the target. The projection $G(\mathcal{O}) \cdot \gamma \mapsto G(\mathcal{O}) \cdot \lambda \subset G(\mathcal{K}) / G(\mathcal{O})$ is a bijection.

\subsection{Positively folded one-skeleton galleries}

Consider a vertex $V$ of a gallery together with the two edges $E$ and $F$. To simplify the notation, we call such a sequence $\left(V_{0} \subset E \supset V \subset F \supset V_{1}\right)$ of vertices and edges a two steps gallery. Note that none of the vertices needs to be a special vertex, and we often omit $V_{0}$ and $V_{1}$. A two steps gallery is called minimal if there exists a sector $\mathfrak{s}$ with vertex $V_{0}$ such that $E \subset \mathfrak{s}$ and $F \subset \mathfrak{s}(V)$. An equivalent condition is the following: there exists a sector $\mathfrak{s}^{\prime}$ with vertex $V$ such that $E \subset \mathfrak{s}^{\prime}$ and $F \subset-\mathfrak{s}^{\prime}$.

Definition 10. We say that a two steps gallery $\left(E \supset V \subset F^{\prime}\right) \subset \mathbb{A}$ is obtained from $(E \supset V \subset F) \subset \mathbb{A}$ by a positive folding if there exists an affine root $(\beta, n)$ such that

$$
V \in \mathrm{H}_{\beta, n}, \quad F^{\prime}=s_{\beta, n}(F) \quad \text { and } \quad \mathrm{H}_{\beta, n} \text { separates } F \text { and } C^{-}(V) \text { from } F^{\prime} .
$$

A two steps gallery $(E \supset V \subset F)$ in $\mathbb{A}$ is called positively folded if either the gallery is a minimal, or if there exist faces $F_{0}, \ldots, F_{s}$ containing $V$ such that:

- $\left(E \supset V \subset F_{0}\right)$ is minimal and $F_{s}=F$,

- $\forall j=1, \ldots, s:\left(E \supset V \subset F_{j}\right)$ is obtained from $\left(E \supset V \subset F_{j-1}\right)$ by a positive folding.

In the residue building at a vertex $V$ we say that $\left(E_{V}, F_{V}\right)$ is a minimal pair if there exists two opposite sectors $\mathfrak{s}$ and $-\mathfrak{s}$ with vertex $V$ such that $E \subset \mathfrak{s}$ and $F \subset-\mathfrak{s}$. We use this notion to get the following equivalent definition for a positively folded two-step gallery, which uses more the language of the residue building:

Definition 11. The two-step gallery $(E \supset V \subset F)$ in $\mathbb{A}$ is called positively folded if there exist

- faces $F_{0, V}, \ldots, F_{s, V}$ such that $\left(E_{V}, F_{0, V}\right)$ is a minimal pair, and $F_{s, V}=F_{V}$,

- for all $j=1, \ldots, s$ there exists an affine $\operatorname{root}\left(\beta_{j}, n_{j}\right)$ such that $\beta_{j} \in \Phi_{V}, V \in \mathrm{H}_{\beta_{j}, n_{j}}$, $s_{\beta_{j}, n_{j}}\left(F_{j-1, V}\right)=F_{j, V}$ and $\mathrm{H}_{\beta_{j}, n_{j}}$ separates $C_{V}^{-}$and $F_{j-1, V}$ from $F_{j, V}$.

Remarks 3. 1) Note that two faces $E_{V}$ and $F_{V}^{\prime}$ could be opposite in $\mathcal{J}_{V}^{\mathfrak{a}}$ (i.e. there exists two opposite chambers $D$ and $-D$ such that $E_{V} \subset D$ and $F_{V}^{\prime} \subset-D$ ) without being a minimal pair. This can be seen in a root system of type $B_{2}$.

2) Note that neither the face $F_{0}$ nor the sequence of reflections are unique in Definition 10 and Definition 11. Below is an example for a root system of type $B_{2}$. The dot in the middle is the vertex $V$. The fact that $(E \supset V \subset F)$ is positively folded can be seen using one of the two faces $F_{0}$ and some reflections with respect to the drawn walls. 


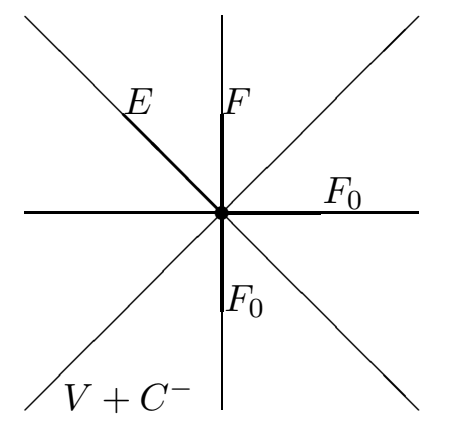

Since the equivalence classes of sectors are in bijection with the Weyl chambers, we can endow the set of equivalence classes with the Bruhat order: $\underline{\mathfrak{s}} \geq \underline{\mathfrak{s}}^{\prime}$ iff $\underline{\mathfrak{s}}=\underline{\tau\left(C^{+}\right)}, \underline{\mathfrak{s}}=\kappa\left(C^{+}\right)$ and $\tau \geq \kappa$. Minimal galleries $\gamma$ are characterized by the property that one can find an associated chain of sectors $\underline{\mathfrak{s}}(\gamma)=\left(\mathfrak{s}_{0}, \ldots \mathfrak{s}_{r}\right)$ such that we have for the classes: $\underline{\mathfrak{s}}_{0}=\ldots=\underline{\mathfrak{s}}_{r}$.

We are going to weaken this condition for the combinatorial positively folded one-skeleton galleries:

Definition 12. For a dominant coweight $\lambda$, let $\gamma_{\lambda}$ be a minimal one-skeleton gallery contained in $C^{+}$, starting in $\mathfrak{o}$ and ending in $\lambda$. A combinatorial one-skeleton gallery of type $t_{\gamma_{\lambda}}$ :

$$
\gamma=\left(V_{0}=\mathfrak{o} \subset E_{0} \supset \ldots \subset E_{r} \supset V_{r+1}\right) \subset \mathbb{A}
$$

is called globally positively folded or just positively folded if

i) the gallery is locally positively folded, i.e. the two-step galleries $\left(E_{i-1} \supset V_{i} \subset E_{i}\right)$ are positively folded for all $i=1, \ldots, r$;

ii) there exists a chain of sectors $\underline{\mathfrak{s}}(\gamma)=\left(\mathfrak{s}_{0}, \ldots \mathfrak{s}_{r}\right)$ such that for all $i=0, \ldots, r$ : $V_{i}$ is the vertex of $\mathfrak{s}_{i}$ and $E_{i} \subset \mathfrak{s}_{i}$, and $\underline{\mathfrak{s}}_{0} \geq \ldots \geq \underline{\mathfrak{s}}_{r}$.

The sequence of sectors respectively the sequence of Weyl group elements $\operatorname{def}(\gamma)=\left(\tau_{0}, \ldots, \tau_{r}\right)$ (where $\underline{\tau_{i}\left(C^{+}\right)}=\underline{\mathfrak{s}}_{i}$ ) is called a defining chain for $\gamma$.

Remark 4. A defining chain for a gallery is not necessarily unique. If $\tau_{0}=\ldots=\tau_{r}$, then the gallery is obviously minimal. Note that the gallery $\gamma$ in Example 8 is locally minimal and hence locally positively folded, i.e. the two-step galleries $\left(E_{0} \supset V_{1} \subset E_{1}\right)$ and $\left(E_{1} \supset V_{2} \subset E_{2}\right)$ are positively folded, but the gallery is not globally positively folded.

\subsection{Local and global properties in special cases}

By Remark 4 and Example 8 we see that minimality and being positively folded are in general not local properties. In this section we will show now that there are many interesting cases where actually local minimality implies global minimality and locally positively folded implies globally positively folded.

Fix a dominant coweight $\lambda$ and let $\gamma_{\underline{\lambda}}$ be a concatenation of the galleries $\gamma_{\omega}$ associated to the fundamental coweights as in Example 3. More precisely, recall that we fixed a total order on the set of fundamental coweights: $\omega_{1}, \ldots, \omega_{n}$, and if $\lambda=\sum a_{i} \omega_{i}$, the associated minimal gallery $\gamma_{\underline{\lambda}}$ is the concatenation of the correspondingly displayed galleries, see Example 3;

$$
\begin{aligned}
\gamma_{\underline{\lambda}} & =\underbrace{\gamma_{\omega_{1}} * \cdots * \gamma_{\omega_{1}}}_{a_{1} \text { times }} * \cdots * \underbrace{\gamma_{\omega_{n}} * \cdots * \gamma_{\omega_{n}}}_{a_{n} \text { times }} \\
& =\left(0=V_{0}^{c} \subset E_{0}^{c} \supset V_{1}^{c} \subset E_{1}^{c} \supset \cdots \supset V_{r}^{c} \subset E_{r}^{c} \supset V_{r+1}^{c}=\lambda\right),
\end{aligned}
$$


where the $V_{j}^{c}$ 's and the $E_{j}^{c}$ 's are vertices and faces of the dominant Weyl chamber.

Let $\operatorname{supp} \lambda$ be the set of nodes $N_{i}$ of the Dynkin diagram such that $a_{i} \neq 0$. We make a special assumption on the enumeration of the nodes:

(*) If $N_{i} \in \operatorname{supp} \lambda$, then none of the nodes $\left\{N_{j} \mid j<i\right\}$ is connected in the Dynkin diagram with one of the nodes $\left\{N_{j} \mid j>i\right\}$.

If the Dynkin diagram has no branches, i.e. the root system of $G$ is of type $\mathrm{A}, \mathrm{B}, \mathrm{C}, \mathrm{F}_{4}$ or $\mathrm{G}_{2}$, then the Bourbaki enumeration of the nodes satisfies the property $(*)$ for all dominant coweights. If $G$ is of type D or $\mathrm{E}$ and $\operatorname{supp} \lambda$ is contained in a subdiagram of type A, then it is easy to see that one can find an enumeration satisfying the condition $(*)$.

Proposition 9. Suppose the enumeration of the nodes of the Dynkin diagram of G satisfies the condition $(*)$ for supp $\lambda$. Let $\gamma \subset \mathbb{A}$ be a combinatorial one-skeleton gallery of the same type as $\gamma_{\underline{\underline{\lambda}}}$. If $\gamma$ is locally positively folded, then $\gamma$ is globally positively folded.

Remark 5. A locally positively folded combinatorial one-skeleton can be viewed as the gallery version of a weakly standard Young tableau defined by Lakshmibai, Musili and Seshadri in [14], §12. The proof below is an adaption of their proof that in special cases (like the ones above) weakly standard Young tableaux are standard Young tableaux.

Proof. Let $\gamma$ be a combinatorial one-skeleton gallery of the same type as $\gamma_{\underline{\lambda}}$, say

$$
\gamma=\left(V_{0}=\mathfrak{o} \subset E_{0} \supset \ldots \subset E_{r} \supset V_{r+1}\right) \subset \mathbb{A} .
$$

The gallery $\gamma$ is a concatenation $\gamma=\gamma_{1} * \ldots * \gamma_{N}$ of combinatorial one-skeleton galleries, each being of the same type as $\gamma_{\omega}$ for some fundamental weight $\omega$ corresponding to one of the nodes in the support of $\lambda$. By abuse of notation we say that an edge $E_{i}$ is of weight type $\omega_{E_{i}}$ if $E_{i}$ occurs in the concatenation within a one-skeleton gallery of the same type as $\gamma_{\omega_{E_{i}}}$, and we say that $E_{i}$ is of weight class $\kappa_{i} \in W / W_{\omega_{E_{i}}}$ if the ray $\mathbb{R}_{\geq 0} \kappa_{i}\left(\omega_{E_{i}}\right)$ coincides with the ray starting in $V_{i}$ and passing through $V_{i+1}$, up to a displacement by $V_{i}$. The gallery $\gamma$ is hence completely described by the sequence of Weyl group classes $\left(\kappa_{0}, \ldots, \kappa_{r}\right)$. Further, given a sector $\mathfrak{s}$ with vertex $V_{i}$, then $E_{i} \subset \mathfrak{s}$ only if $\underline{\mathfrak{s}}=\tau\left(C^{+}\right)$for an element $\tau \in W$ such that $\tau \equiv \kappa_{i} \bmod W_{\omega_{E_{i}}}$.

It follows that to give a sequence of sectors $\left(\mathfrak{s}_{0}, \ldots, \mathfrak{s}_{r}\right)$ such that $\mathfrak{s}_{i}$ has vertex $V_{i}$ and $E_{i} \subset \mathfrak{s}$ is equivalent to give a sequence of Weyl group elements $\left(\tau_{0}, \ldots, \tau_{r}\right)$ such that $\tau_{i} \equiv \kappa_{i} \bmod W_{\omega_{E_{i}}}$ for $i=0, \ldots, r$. The gallery is globally positively folded if and only if one can choose the Weyl group elements such that in addition $\tau_{0} \geq \ldots \geq \tau_{r}$.

As a first step, we show that the local minimality implies for all $i=0, \ldots, r-1$ the existence of pairs $\left(\sigma_{i}, \eta_{i+1}\right) \in W \times W$ such that $\sigma_{i} \geq \eta_{i+1}, \sigma_{i} \equiv \kappa_{i} \bmod W_{\omega_{E_{i}}}$ and $\eta_{i+1} \equiv$ $\kappa_{i+1} \bmod W_{\omega_{E_{i+1}}}$. For the positively folded two-step gallery $\left(E_{i} \supset V_{i+1} \subset E_{i+1}\right)$ let $\left(E_{i} \supset\right.$ $\left.V_{i+1} \subset F_{0}\right)$ be a corresponding minimal gallery with sector $\mathfrak{t}_{0}$, i.e., $\mathfrak{t}_{0}$ has vertex $V_{i}, E_{i} \subset \mathfrak{t}_{0}$ and $F_{0} \subset \mathfrak{t}_{0}^{\prime}=\mathfrak{t}_{0}\left(V_{i+1}\right)$. If $F_{0}=E_{i+1}$, then set $\mathfrak{t}_{1}=\mathfrak{t}_{0}^{\prime}$. If $F_{0} \neq E_{i+1}$, then let $(\beta, n)$ be the affine root such that $F_{1}=s_{\beta, n}\left(F_{0}\right)$ is obtained by a positive folding. Since $\mathrm{H}_{\beta, n}$ separates $F_{0}$ and $C^{-}(V)$ from $F_{1}$, it separates also $\mathfrak{t}_{0}^{\prime}$ and $C^{-}(V)$ from $\mathfrak{t}_{1}^{\prime}=s_{\beta, n}\left(\mathfrak{t}_{0}^{\prime}\right)$, so $\underline{\mathfrak{t}}_{0} \geq \underline{\mathfrak{t}}_{1}^{\prime}$. By repeating the argument if $E_{i+1} \neq F_{1}$, we obtain successively the sector $\mathfrak{t}_{1}$ with vertex $V_{i+1}$ such that $E_{i+1} \subset \mathfrak{t}_{1}$ and $\underline{\mathfrak{t}}_{0} \geq \underline{\mathfrak{t}}_{1}$. Let $\sigma_{i}, \eta_{i+1} \in W$ be such that $\sigma_{i}\left(C^{+}\right)=\underline{\mathfrak{t}}_{0}$ and $\eta_{i+1}\left(C^{+}\right)=\underline{\mathfrak{t}}_{1}$, so $\sigma_{i} \geq \eta_{i+1}$ and $\sigma_{i} \equiv \kappa_{i} \bmod W_{\omega_{E_{i}}}, \eta_{i+1} \equiv \kappa_{i+1} \bmod W_{\omega_{E_{i+1}}}$.

We start now to define the sequence of Weyl group elements $\tau_{0}, \ldots, \tau_{r}$ by choosing for $\tau_{0} \in$ $W$ the maximal representative of the class $\kappa_{0}$. Suppose we have already defined $\tau_{0}, \ldots, \tau_{i} \in W$ 
such that $\tau_{0} \geq \ldots \geq \tau_{i}$ and $\tau_{j} \equiv \kappa_{j} \bmod W_{\omega_{E_{j}}}$ for $j=0, \ldots, i$. Let $k_{0}$ be such that the node $N_{k_{0}}$ corresponds to the fundamental weight $\omega_{E_{i}}$, let $I$ be the set of nodes $I=\left\{N_{\ell} \mid \ell<k_{0}\right\}$ and set $J=\left\{N_{\ell} \mid \ell>k_{0}\right\}$. Denote by $W_{I}, W_{J}$ and $W_{I \cup J}$ the subgroups of $W$ generated by the $s_{\alpha}$ associated to the simple roots corresponding to the nodes in $I, J$ and $I \cup J$ respectively. The condition $(*)$ implies that the elements in $W_{I}$ commute with the elements in $W_{J}$. By abuse of notation we write $\bar{\tau}_{j}$ not only for the class of $\tau_{j}$ in $W / W_{\omega_{E_{i}}}=W / W_{I \cup J}$, but also for the minimal representative of this class in $W$. So we can write $\tau_{j}=\bar{\tau}_{j} x_{j} y_{j}$, where $x_{j} \in W_{I}$ and $y_{j} \in W_{J}$. Recall that $x_{j} y_{j}=y_{j} x_{j}$ by condition $(*)$.

Since $\bar{\tau}_{j} x_{j}$ is a minimal representative in $W$ of the class $\left(\tau_{j} \bmod W_{J}\right)$, the inequalities $\tau_{0} \geq \ldots \geq \tau_{i}$ imply the inequalities $\bar{\tau}_{0} x_{0} \geq \ldots \geq \bar{\tau}_{i} x_{i}$. Let now $y$ be the maximal element in $W_{J}$. Since $y$ and the $y_{0}, \ldots, y_{i}$ fix the fundamental weight $\omega_{\ell}$ for $\ell<k_{0}$, we can assume without loss of generality $y_{j}=y$ for all $j=0, \ldots, i$, because if one replaces the $y_{j}$ by $y$, then one still has the desired properties for all $j=0, \ldots, i$ :

$$
\tau_{0}=\bar{\tau}_{0} x_{0} y \geq \tau_{1}=\bar{\tau}_{1} x_{1} y \geq \ldots \geq \tau_{i}=\bar{\tau}_{i} x_{i} y, \quad \text { and } \quad \tau_{j} \equiv \kappa_{j} \quad \bmod W / W_{\omega_{E_{j}}} .
$$

To extend the sequence and define $\tau_{i+1}$, we consider now the pair $\sigma_{i} \geq \eta_{i+1}$ defined at the beginning. Recall that $\sigma_{i} \equiv \kappa_{i}=\bar{\tau}_{i} \bmod W_{\omega_{E_{i}}}, \eta_{i+1} \equiv \kappa_{i+1} \bmod W_{\omega_{E_{i+1}}}$. We can write $\sigma_{i}=\bar{\tau}_{i} p_{i} q_{i}$ and $\eta_{i+1}=\bar{\eta}_{i+1} r_{i+1} t_{i+1}$, where $p_{i}, r_{i+1} \in W_{I}, q_{i}, t_{i+1} \in W_{J}$ and $\bar{\eta}_{i+1}$ denotes the class of $\eta_{i+1}$ in $W / W_{I \cup J}$ as well as the minimal representative of the class in $W$.

Set $\tau_{i+1}=\bar{\eta}_{i+1} t_{i+1}$, then $\tau_{i+1} \equiv \kappa_{i+1} \bmod W_{\omega_{E_{i+1}}}$ because $r_{i+1}$ fixes $\omega_{E_{i+1}}$. Further, $\tau_{i}=\bar{\tau}_{i} x_{i} y \geq \tau_{i+1}=\bar{\eta}_{i+1} t_{i+1}$ because

$$
\left(\sigma_{i} \bmod W_{\omega_{E_{i}}}\right)=\kappa_{i}=\bar{\tau}_{i} \geq\left(\eta_{i+1} \bmod W_{\omega_{E_{i}}}\right)=\bar{\eta}_{i+1}
$$

and, by construction, $y \geq t_{i+1}$. Proceeding by induction gives the desired defining chain.

\subsection{Semistandard Young tableaux and positively folded one-skeleton gal- leries}

To characterize the tableaux corresponding to positively folded galleries, recall that the Bourbaki enumeration of the fundamental coweights satisfies the condition $(*)$ in section 5.4 for the groups of type $A_{n}, B_{n}$ and $C_{n}$.

Proposition 10. The bijection in Proposition 1 induces a bijection between the positively folded galleries and the semistandard tableaux.

Proof. By Proposition 9, a locally positively folded gallery is automatically globally positively folded. Consider two consecutive faces of dimension one in the gallery: $\left(V_{i-1} \subset E_{i-1} \supset V_{i} \subset\right.$ $\left.E_{i}\right)$. Then either $E_{i-1}=V_{i-1}+\sigma\left(\left\{t \omega_{j} \mid t \in[0,1]\right\}\right)$ or $E_{i-1}=V_{i-1}+\sigma\left(\left\{t \omega_{j} \mid t \in\left[0, \frac{1}{2}\right]\right\}\right)$ for some $j$ and some $\sigma \in W / W_{\omega_{j}}$, and $E_{i}=V_{i}+\tau\left(\left\{t \omega_{k} \mid t \in[0,1]\right\}\right)$ or $E_{i}=V_{i}+\tau\left(\left\{t \omega_{k} \mid\right.\right.$ $\left.t \in\left[0, \frac{1}{2}\right]\right\}$ ) for $k=j$ or $k=j+1$ and some $\tau \in W / W_{\omega_{k}}$ (for a more precise description of the possible $\tau$ in the second case, see Equation (8) ). Denote by $C_{i-1}, C_{i}$ the columns in the Young tableaux corresponding to the weights $\sigma\left(\omega_{j}\right)$ and $\tau\left(\omega_{k}\right)$. It remains to show that the condition positively folded at $V_{i}$ is equivalent to the condition that the entries in the rows of the tableaux consisting of the two columns $C_{i-1}$ (on the right side) and $C_{i}$ are weakly increasing.

It is easy to verify that the condition on the rows is equivalent to $\sigma \geq \tau$ in $W / W_{\omega_{j}}$ if $\omega_{j}=\omega_{k}$ (see [2], Chapter 3), respectively there exists lifts $\tilde{\sigma} \in W$ of $\sigma$ and $\tilde{\tau} \in W$ of $\tau$ such 
that $\tilde{\sigma} \geq \tilde{\tau}$ (see [14]). Suppose first $V_{i}$ is a special point. The condition $\tilde{\sigma} \geq \tilde{\tau}$ implies the condition positively folded: one starts with the sector $V_{i}+\tilde{\sigma}\left(C^{+}\right)$which contains a conjugate of $E_{i}$ forming a minimal pair with $E_{i-1}$. If $\tilde{\sigma}>\tilde{\tau}$, then one can find a sequence of reflections such that $\tilde{\sigma}>s_{\beta_{1}} \tilde{\sigma}>\ldots>\tilde{\tau}$ and the length decreases in each step by one. It follows that the corresponding folds at $V_{i}$ are all positive. The reverse direction is proved in the same way: start with a minimal pair $E_{i-1} \supset V_{i} \subset E_{i, 0}$ for $E_{i-1} \supset V_{i} \subset E_{i}$, let $\tilde{\sigma}\left(C^{+}\right)$be the chamber such that $E_{i-1} \subset V_{i-1}+\tilde{\sigma}\left(C^{+}\right)$and $E_{i, 0} \subset V_{i}+\tilde{\sigma}\left(C^{+}\right)$, applying the positive folds to the sector $V_{i}+\tilde{\sigma}\left(C^{+}\right)$yields a sector $V_{i}+\tilde{\tau}\left(C^{+}\right)$containing $E_{i}$, and, since the folds are positive, one has $\tilde{\sigma} \geq \tilde{\tau}$. Since the sectors contain the faces we have $\tilde{\sigma}\left(\omega_{j}\right)=\sigma\left(\omega_{j}\right)$ and $\tilde{\tau}\left(\omega_{k}\right)=\tau\left(\omega_{k}\right)$, i.e. these are lifts for $\sigma$ and $\tau$. If $V_{i}$ is not a special point, then $W_{V_{i}}^{v}=\left(W_{V_{i}}^{1}\right)^{v} \times\left(W_{V_{i}}^{2}\right)^{v}$ is of type $\mathrm{B}_{j} \times \mathrm{B}_{n-j}$ or $\mathrm{D}_{j} \times \mathrm{C}_{j}$, where, in both cases, the second factor acts trivially. We have a bijection between the possible entries of $C_{i}$ and the orbit $\left(W_{V_{i}}^{1}\right)^{v} \cdot\left(\epsilon_{1}+\epsilon_{2}+\cdots+\epsilon_{j}\right)$, in the following way: Let $k_{1}, \ldots, k_{s}, \bar{\ell}_{1}, \ldots, \bar{\ell}_{j-s}, 1 \leq k_{p}, \ell_{q} \leq n$, be the entries of $C_{i-1}$, we order the set of integers $\left\{k_{1}, \ldots, k_{s}, \ell_{1}, \ldots, \ell_{j-s}\right\}$ in ascending order and we identify this linearly ordered set with $\{1,2, \ldots, j\}$. With respect to this bijection, the columns $C_{i-1}, C_{i}$ correspond to $\sigma\left(\epsilon_{1}+\epsilon_{2}+\cdots+\epsilon_{j}\right)$ and $\tau\left(\epsilon_{1}+\epsilon_{2}+\cdots+\epsilon_{j}\right)$ for some $\sigma, \tau \in\left(W_{V_{i}} 1\right)^{v} /\left(W_{V_{i}} 1\right)_{\epsilon_{1}+\cdots+\epsilon_{j}}^{v}$. Now again (see [2]), the Bruhat order on the orbit and the row condition on pairs of colums coincide. So the same arguments, as above, show that positively folded and the row condition are equivalent.

\section{Local minimality}

The language of building theory allows us to translate the study of the intersection $Z_{\lambda, \mu}:=$ $G(\mathcal{O}) . \lambda \cap U^{-}(\mathcal{K}) . \mu$ into a problem of studying intersections of subsets of a Bott-Samelson variety $\Sigma\left(\gamma_{\lambda}\right)$ :

$$
Z_{\lambda, \mu}=G(\mathcal{O}) \cdot \lambda \cap U^{-}(\mathcal{K}) \cdot \mu=\bigcup_{\substack{\delta \in \Gamma\left(t_{\gamma_{\lambda}}, \mathfrak{o}\right) \\ \operatorname{target}(\delta)=\mu}}\{\text { minimal galleries }\} \cap C_{\delta} .
$$

Here $C_{\delta}$ denotes the Białynicki-Birula cell associated to the combinatorial gallery $\delta$, which, in terms of building theory, is the same as the fiber over $\delta$ of the retraction $r_{-\infty}$.

To describe more precisely the intersection of the set of minimal galleries with such a cell, we need to "unfold" $\delta$, i.e. we need to construct minimal galleries that retract onto $\delta$. As a first step we will, in this section, describe how to unfold two steps galleries. An important tool will be the galleries of residue chambers.

\subsection{Positively folded galleries of chambers}

Let $E$ and $F$ be one dimensional faces in $\mathcal{J}^{\mathfrak{a}}$ containing a vertex $V$, let also $\mathfrak{s}$ be a sector with vertex $V$ containing $E$. Let $w_{\mathfrak{s}_{V}}=w\left(C_{V}^{-}, \mathfrak{s}_{V}\right)$ be the element in $W_{V}^{v}$ that sends $C_{V}^{-}$to $\mathfrak{s}_{V}$. Among the residue chambers containing $F_{V}$ denote by $D$ the one closest to $C_{V}^{-}$. Fix a reduced decomposition of $w_{F}=w\left(C_{V}^{-}, D\right)=s_{i_{1}} \cdots s_{i_{r}}$ in $W_{V}^{v}$ and let $\mathbf{i}=\left(i_{1}, \ldots, i_{r}\right)$ be the type of the decomposition. We denote by $\alpha_{i_{j}}$ the simple root in $\Phi_{V}$ corresponding to $s_{i_{j}}$. For any root $\alpha \in \Phi_{V}, x_{\alpha}(\cdot)$ denotes the one-parameter additive subgroup of $H_{V}$ associated to $\alpha$, let $U_{\alpha}$ denote its image in $H_{V}$.

We consider now galleries of residue chambers $\mathbf{c}=\left(C_{V}^{-}, C_{1}, \ldots, C_{r}\right)$ in the apartment $\mathbb{A}_{V}$ starting at $C_{V}^{-}$and of type $\mathbf{i}$. The set of these galleries is in bijection with the set 
$\Gamma(\mathbf{i})=\left\{1, s_{i_{1}}\right\} \times \cdots \times\left\{1, s_{i_{r}}\right\}$ via the $\operatorname{map}\left(c_{1}, \ldots, c_{r}\right) \mapsto\left(C_{V}^{-}, c_{1} C_{V}^{-}, \ldots, c_{1} \cdots c_{r} C_{V}^{-}\right)$. Let $\beta_{j}=c_{1} \cdots c_{j}\left(\alpha_{i_{j}}\right)$, then $\beta_{j}$ is the root corresponding to the common wall $H_{j}=H_{\beta_{j}}$ of $C_{j-1}=c_{1} \cdots c_{j-1} C_{V}^{-}$and $C_{j}=c_{1} \cdots c_{j} C_{V}^{-}$. In the following, we shall identify a sequence $\left(c_{1}, \ldots, c_{r}\right)$ and the corresponding gallery.

Definition 13. A gallery $\mathbf{c}=\left(c_{1}, \ldots, c_{r}\right) \in \Gamma(\mathbf{i})$ is said to be positively folded with respect to $\mathfrak{s}_{V}$ if $c_{j}=1$ implies $w_{\mathfrak{s}_{V}}^{-1} \beta_{j}<0$. We denote the set of positively folded galleries by $\Gamma_{\mathfrak{s}_{V}}^{+}(\mathbf{i})$.

If $\mathfrak{s}_{V}=C_{V}^{+}$, a gallery $\mathbf{c}=\left(c_{1}, \ldots, c_{r}\right)$ is positively folded with respect to $C_{V}^{+}$if, and only if, the associated subexpression $\left(i d, c_{1}, c_{1} c_{2}, \ldots, c_{1} \cdots c_{r}\right)$ is distinguished, see Deodhar [8], Definition 2.3.

Proposition 11. A gallery $\mathbf{c}=\left(C_{V}^{-}, C_{1}, \ldots, C_{r}\right) \in \Gamma(\mathbf{i})$ is positively folded with respect to $\mathfrak{s}_{V}$ if, and only if, $C_{j}=C_{j-1}$ implies that the wall $H_{j}=H_{\beta_{j}}$ separates $\mathfrak{s}_{V}$ from $C_{j}=C_{j-1}$.

Proof. We have the following equivalences:

$\left(H_{j}\right.$ separates $\mathfrak{s}_{V}$ from $\left.C_{j}=C_{j-1}\right) \Longleftrightarrow\left(w_{\mathfrak{s}_{V}}^{-1} H_{j}\right.$ separates $C_{V}^{-}$from $\left.w_{\mathfrak{s}_{V}}^{-1} C_{j}=w_{\mathfrak{s}_{V}}^{-1} C_{j-1}\right) \Longleftrightarrow$ $\left(w_{\mathfrak{s}_{V}}^{-1} \beta_{j}\right.$ is a negative root).

The set of all galleries of chambers starting at $C_{V}^{-}$of type $\mathbf{i}$ in the building $\mathcal{J}_{V}^{\mathfrak{a}}$ has a structure of a smooth projective algebraic variety, which we denote by $\mathrm{BS}(\mathbf{i})$. (In fact, it is a Bott-Samelson variety.) To a gallery of chambers $\mathbf{c}=\left(c_{1}, \ldots, c_{r}\right)=\left(C_{V}^{-}, C_{1}, \ldots, C_{r}\right)$ in $\Gamma(\mathbf{i})$, one can associate an open subset $\mathcal{O}_{\mathfrak{s}_{V}}(\mathbf{c})$ and a cell $\mathcal{C}_{\mathfrak{s}_{V}}(\mathbf{c})$ in the variety $\mathrm{BS}(\mathbf{i})$. They are defined in the following way: for any $j \in\{1, \ldots, r\}$, and any $a_{j} \in \mathbb{C}$, set $o_{j}=x_{c_{j}\left(\alpha_{i_{j}}\right)}\left(a_{j}\right) c_{j}$, then $\mathcal{O}_{\mathfrak{s}_{V}}(\mathbf{c})=\left\{\left(C_{V}^{-}=C_{0}^{\prime}, C_{1}^{\prime}, \ldots, C_{r}^{\prime}\right) \mid \forall j: C_{j}^{\prime}=o_{1} \cdots o_{j} C_{V}^{-}\right\}$; further, set

$$
g_{j}= \begin{cases}c_{j} & \text { if } w_{\mathfrak{s}_{V}}^{-1} \beta_{j}>0 \\ x_{c_{j}\left(\alpha_{i_{j}}\right)}\left(a_{j}\right) c_{j} & \text { if } w_{\mathfrak{s}_{V}}^{-1} \beta_{j}<0\end{cases}
$$

then $\mathcal{C}_{\mathfrak{s}_{V}}(\mathbf{c})=\left\{\left(C_{V}^{-}=C_{0}^{\prime}, C_{1}^{\prime}, \ldots, C_{r}^{\prime}\right) \mid \forall j: C_{j}^{\prime}=g_{1} \cdots g_{j} C^{-}\right\}$. The minimal galleries in $\mathcal{C}_{\mathfrak{s}_{V}}(\mathbf{c})$ are those such that for any $j: C_{j-1}^{\prime} \neq C_{j}^{\prime}$, i.e. $c_{j} \neq 1$ if $w_{\mathfrak{s}_{V}}^{-1} \beta_{j}>0$, and $a_{j} \neq 0$ if $c_{j}=1$ and $w_{\mathfrak{s}_{V}}^{-1} \beta_{j}<0$. We denote the set of minimal galleries by $\mathcal{C}_{\mathfrak{s}_{V}}^{m}(\mathbf{c})$.

Lemma 4. The set $\mathcal{C}_{\mathfrak{s}_{V}}^{m}(\mathbf{c})$ is empty if the gallery $\mathbf{c}$ is not positively folded with respect to $\mathfrak{s}_{V}$. If $\mathbf{c}$ is positively folded with respect to $\mathfrak{s}_{V}$, then $\mathcal{C}_{\mathfrak{s}_{V}}^{m}(\mathbf{c})$ is isomorphic to:

$$
\mathcal{C}_{\mathfrak{s}_{V}}^{m}(\mathbf{c}) \simeq \mathbb{C}^{t(\mathbf{c})} \times\left(\mathbb{C}^{*}\right)^{r(\mathbf{c})}
$$

where

$$
t(\mathbf{c})=\sharp\left\{j \mid c_{j}=s_{i_{j}} \text { and } w_{\mathfrak{s}_{V}}^{-1} \beta_{j}<0\right\}, r(\mathbf{c})=\sharp\left\{j \mid c_{j}=1 \text { and } w_{\mathfrak{s}_{V}}^{-1} \beta_{j}<0\right\} .
$$

Proposition 12. The cell $\mathcal{C}_{\mathfrak{s}_{V}}(\mathbf{c})$ identifies with $r_{\mathfrak{s}_{V}}^{-1}(\mathbf{c})$, where $r_{\mathfrak{s}_{V}}: \mathcal{J}_{V}^{\mathfrak{a}} \rightarrow \mathbb{A}_{V}$ is the retraction centered at $\mathfrak{s}_{V}$.

Proof. For any chamber $C^{\prime}$, the retraction can be defined as $r_{\mathfrak{s}_{V}}\left(C^{\prime}\right)=\lim _{s \rightarrow 0} s^{\theta} C^{\prime}$, where $\theta$ is a regular coweight contained in $\mathfrak{s}_{V}$. To simplify, we take $\theta=w_{\mathfrak{s}_{V}}\left(-\rho^{\vee}\right)$. Further, the retraction applies componentwise to the galleries, whence $r_{\mathfrak{s}_{V}}(\mathbf{g})=\left(C_{V}^{-}, r_{\mathfrak{s}_{V}}\left(C_{1}^{\prime}\right), \ldots, r_{\mathfrak{s}_{V}}\left(C_{r}^{\prime}\right)\right)$. For any $j, r_{\mathfrak{s}_{V}}\left(C_{j}^{\prime}\right)=\lim _{s \rightarrow 0} s^{w_{\mathfrak{s}_{V}}}\left(-\rho^{\vee}\right) g_{1} \cdots g_{j} C_{V}^{-}=\lim _{s \rightarrow 0} g_{1}^{\prime} \cdots g_{j}^{\prime} C_{V}^{-}$, where

$$
g_{j}^{\prime}= \begin{cases}c_{j} & \text { if } w_{\mathfrak{s}_{V}}^{-1} \beta_{j}>0 \\ x_{c_{j}\left(\alpha_{i_{j}}\right)}\left(s^{\left\langle c_{j}\left(\alpha_{i_{j}}\right), c_{j-1} \cdots c_{1} w_{\mathfrak{s}_{V}}\left(-\rho^{\vee}\right)\right\rangle} a_{j}\right) c_{j} & \text { if } w_{\mathfrak{s}_{V}}^{-1} \beta_{j}<0 .\end{cases}
$$


But $\left\langle c_{j}\left(\alpha_{i_{j}}\right), c_{j-1} \cdots c_{1} w_{\mathfrak{s}_{V}}\left(-\rho^{\vee}\right)\right\rangle=\left\langle w_{\mathfrak{s}_{V}}^{-1} \beta_{j},-\rho^{\vee}\right\rangle$. Therefore, $\mathcal{C}_{\mathfrak{s}_{V}}(\mathbf{c}) \subset r_{C_{\mathfrak{s}_{V}}}^{-1}(\mathbf{c})$. One sees in the same way that $\mathcal{C}_{\mathfrak{s}_{V}}(\mathbf{c}) \supset r_{C_{\mathfrak{s}_{V}}}^{-1}(\mathbf{c})$.

Remark 6. The cells define a Białynicki-Birula decomposition of the variety of all galleries of chambers BS(i). In fact, $\mathrm{BS}(\mathbf{i})=\coprod_{\mathbf{c} \in \Gamma(\mathbf{i})} \mathcal{C}_{\mathfrak{s}_{V}}(\mathbf{c})$.

\subsection{Two steps minimal one-skeleton galleries}

Theorem 1. Let $(E \supset V \subset F)$ be a two steps one-skeleton gallery in $\mathbb{A}$. There exists a minimal gallery $\left(E \supset V \subset E^{\prime}\right)$ in $\mathcal{J}^{\mathfrak{a}}$ such that $E^{\prime}$ has the same type as $F$ and $r_{-\infty}\left(E^{\prime}\right)=F$ if, and only if, $(E \supset V \subset F)$ is positively folded.

We divide the proof of Theorem 1 into four lemmas. Choose a chamber $D$ containing $F_{V}$ and let $w$ be the element that sends $C_{V}^{-}$to $D$.

Lemma 5. Suppose there exists a minimal one-skeleton gallery $\left(E \supset V \subset E^{\prime}\right)$ such that $r_{-\infty}\left(E^{\prime}\right)=F$. Let $\mathfrak{s}$ be a sector in $\mathbb{A}$ with vertex $V$ containing $E$ such that $E^{\prime} \subset-\mathfrak{s}$, in any apartment containing $\mathfrak{s}$ and $E^{\prime}$. Then one can find a minimal gallery of residue chambers $\mathbf{m}^{\prime}$ of type $\mathbf{i}=\left(i_{1}, \ldots, i_{r}\right)$ between $C_{V}^{-}$and $E_{V}^{\prime}$ such that

i) $w=s_{i_{1}} \cdots s_{i_{r}}$ is a reduced decomposition,

ii) $\mathbf{c}=r_{\mathfrak{s}_{V}}\left(\mathbf{m}^{\prime}\right) \subset \mathbb{A}_{V}$ is a positively folded gallery of residue chambers with respect to $\mathfrak{s}_{V}$,

iii) $\left(E_{V}, F_{V}^{\prime}\right)$ is a minimal pair, where $F_{V}^{\prime}=r_{\mathfrak{s}_{V}}\left(E_{V}^{\prime}\right)$ and $F_{V}^{\prime}$ is of the same type as $F_{V}$.

Proof. The fact that $E^{\prime}$ has the same type as $F$ is a consequence of $r_{-\infty}\left(E^{\prime}\right)=F$. Transferred to the setting of the residue building, the retraction $r_{-\infty}$ identifies with the retraction centered at $C_{V}^{-}$of $\mathcal{J}_{V}^{\mathfrak{a}}$ onto $\mathbb{A}_{V}$, so $r_{C_{V}^{-}}\left(E_{V}^{\prime}\right)=F_{V}$. Since this retraction preserves the distances from $C_{V}^{-}$, any minimal gallery $\mathbf{m}^{\prime}=\left(C_{V}^{-}, C_{1}^{\prime}, \ldots, C_{r}^{\prime}\right)$ of residue chambers in $\mathcal{J}_{V}^{\mathfrak{a}}$ from $C_{V}^{-}$to $E_{V}^{\prime}$ (in any apartment containing those two) retracts onto a minimal gallery from $C_{V}^{-}$to $F_{V}$, say of type $\mathbf{i}=\left(i_{1}, \ldots, i_{r}\right)$. Further, one can choose $C_{r}^{\prime}$ such that $r_{C_{V}^{-}}\left(C_{r}^{\prime}\right)=D$. Since the gallery is minimal, it follows that $\mathbf{i}=\left(i_{1}, \ldots, i_{r}\right)$ corresponds to a reduced decomposition of the element $w$.

Consider the variety of galleries $\mathrm{BS}(\mathbf{i})$. The gallery $\mathbf{m}^{\prime}$ belongs to the cell $\mathcal{C}_{\mathfrak{s}_{V}}(\mathbf{c})$, where $\mathbf{c}=r_{\mathfrak{s}_{V}}\left(\mathbf{m}^{\prime}\right)=\left(C_{V}^{-}, C_{1}, \ldots, C_{r}\right)$. Let us suppose that $C_{j}=C_{j-1}$. Let us moreover assume (without loss of generality) that the gallery $\mathbf{m}^{\prime}$ is already retracted until the index $j-1$, meaning that $\mathbf{m}^{\prime}=\left(C_{V}^{-}, C_{1}, \ldots, C_{j-1}, D_{j}, \ldots, D_{r}\right)$, where $\left(C_{j-1}, D_{j}, \ldots, D_{r}\right)$ is a minimal gallery retracting onto $\left(C_{j-1}, C_{j}, \ldots, C_{r}\right)$. Suppose that $H_{j}$ does not separate $\mathfrak{s}_{V}$ from $C_{j}=C_{j-1}$. The chambers $C_{j-1}$ and $D_{j}$ have to be distinct by the assumption on the minimality, so $C_{j}=r_{\mathfrak{s}_{V}}\left(D_{j}\right)$ and $\mathfrak{s}_{V}$ can not be on the same side of $H_{j}$, contradicting the assumption $C_{j}=C_{j-1}$ are not separated from $\mathfrak{s}_{V}$ by $H_{j}$. It follows that the gallery of chambers $\mathbf{c}$ is positively folded, i.e., $\mathbf{c} \in \Gamma_{\mathfrak{s}_{V}}^{+}(\mathbf{i})$.

Let $r_{\infty, \mathfrak{s}}$ be the retraction from $\infty$, but now with respect to the sector $\mathfrak{s}$. On the level of the residue building, the retraction $r_{\infty, \mathfrak{s}}$ identifies with the retraction centered at $\mathfrak{s}_{V}$ of $\mathcal{J}_{V}^{\mathfrak{a}}$ onto $\mathbb{A}_{V}$. So if we set $r_{\infty, \mathfrak{s}}\left(E^{\prime}\right)=F^{\prime}$, then $F_{V}^{\prime}=r_{\mathfrak{s}_{V}}\left(E_{V}^{\prime}\right)$ and we get a minimal pair $\left(E_{V}, F_{V}^{\prime}\right)$ in $\mathcal{J}_{V}^{\mathfrak{a}}$. 
The face $E_{V}^{\prime}$ is contained in the opposite of $\mathfrak{s}_{V}$ in any apartment containing $E_{V}$ and $E_{V}^{\prime}$, and $r_{\mathfrak{s}_{V}}$ preserves the distance from $\mathfrak{s}_{V}$. It follows that $F_{V}^{\prime}=r_{\mathfrak{s}_{V}}\left(E_{V}^{\prime}\right)$ is contained in $-\mathfrak{s}_{V}$, and hence we get a minimal pair $\left(E_{V}, F_{V}^{\prime}\right)$ in $\mathcal{J}_{V}^{\mathfrak{a}}$. Since the type of $E_{V}^{\prime}$ and $F_{V}$ are the same and the type of $E_{V}^{\prime}$ and $F_{V}^{\prime}$ are the same, this finishes the proof of the lemma.

Lemma 6. If there exists a minimal one-skeleton gallery $\left(E \supset V \subset E^{\prime}\right)$ such that $r_{-\infty}\left(E^{\prime}\right)=$ $F$, then the one-skeleton gallery $(E \supset V \subset F)$ is positively folded.

Proof. Let $\mathbf{c}=r_{\mathfrak{s}_{V}}\left(\mathbf{m}^{\prime}\right) \subset \mathbb{A}_{V}$ be the positively folded gallery of residue chambers with respect to $\mathfrak{s}_{V}$ described in Lemma 5. By construction, unfolding $\mathbf{c}$ gives a minimal gallery from $C_{V}^{-}$to $F_{V}$. We will see that this unfolding procedure shows that $(E \supset V \subset F)$ is positively folded.

The procedure works as follows: Let $\left\{j_{1}<\cdots<j_{k}\right\} \subset\{1, \ldots, r\}$ be the indices where c is folded. Then we unfold the gallery of chambers starting with the fold at the wall $H_{j_{1}}$, the resulting gallery will then still have a fold at $s_{H_{j_{1}}}\left(H_{j_{2}}\right)$, we unfold the gallery at this wall etc. The face $F_{V}^{\prime}$ will be reflected each time and we get

$$
\begin{aligned}
F_{V} & =s_{H_{j_{1}}} \cdots s_{H_{j_{k-1}}} s_{H_{j_{k}}}\left(s_{H_{j_{1}}} \cdots s_{H_{j_{k-1}}}\right)^{-1} \cdots s_{H_{j_{1}}} s_{H_{j_{2}}} s_{H_{j_{1}}} s_{H_{j_{1}}} F_{V}^{\prime} \\
& =\tau_{k} \cdots \tau_{1} F_{V}^{\prime}
\end{aligned}
$$

where $\tau_{l}=s_{H_{j_{1}}} \cdots s_{H_{j_{l-1}}} s_{H_{j_{l}}}\left(s_{H_{j_{1}}} \cdots s_{H_{j_{l-1}}}\right)^{-1}$. To see that $(E \supset V \subset F)$ is positively folded, it remains to prove that each time the face is reflected away from $C_{V}^{-}$.

First recall that $\mathbf{c}$ is positively folded, so for each folding step we have the chambers $C_{j_{k}}=C_{j_{k}-1}$ and $\mathfrak{s}_{V}$ lie within different half-spaces with respect to the wall $H_{j_{k}}$. Further, since $F_{V}^{\prime} \subset-\mathfrak{s}_{V}$, the chambers $C_{j_{k}}=C_{j_{k}-1}$ and the face $F_{V}^{\prime}$ lie within the same half-space. We use the suggestive notation

$$
F_{V}^{\prime}, C_{j_{k}}=\left.C_{j_{k}-1} \quad\right|_{H_{j_{k}}} \quad \mathfrak{s}_{V} \supset E_{V}
$$

for this situation.

The gallery of chambers $\mathbf{c}$ starts at $C_{V}^{-}$and is folded for the first time at the hyperplane $H_{j_{1}}$. It follows that the chambers $C_{V}^{-}$and $C_{j_{1}}=C_{j_{1}-1}$, and hence also $F_{V}^{\prime}$, are within the same half-space with respect to $H_{j_{1}}$ :

$$
C_{V}^{-}, C_{j_{k}}=C_{j_{k}-1},\left.F_{V}^{\prime} \quad\right|_{H_{j_{k}}} \quad \mathfrak{s}_{V} \supset E_{V}
$$

Thus, after the first unfolding, we have:

$$
C_{V}^{-}, C_{1}, \ldots,\left.C_{j_{1}-1} \quad\right|_{H_{j_{1}}} \tau_{1}\left(C_{j_{1}}\right), \tau_{1}\left(F_{V}^{\prime}\right)
$$

meaning that the chambers $C_{V}^{-}, C_{1}, \ldots, C_{j_{1}-1}$ are separated from $\tau_{1}\left(C_{j_{1}}\right)$ and from $\tau_{1}\left(F_{V}^{\prime}\right)$ by the wall $H_{j_{1}}$ (note that the face $F_{V}^{\prime}=\tau_{1}\left(F_{V}^{\prime}\right)$ may be contained in the wall $H_{j_{1}}$ ). In particular, either $F_{V}^{\prime}$ is fixed by the reflection or is reflected away from $C_{V}^{-}$. The gallery

$$
\mathbf{c}^{1}=\left(C_{V}^{-}, C_{1}, \ldots, C_{j_{1}-1}, \tau_{1}\left(C_{j_{1}}\right), \ldots, \tau_{1}\left(C_{j_{2}-1}\right), \tau_{1}\left(C_{j_{2}}\right), \ldots, \tau_{1}\left(C_{r}\right)\right)
$$

is now minimal up to the index $j_{2}-1$. Moreover, we know that

$$
F_{V}^{\prime}, C_{j_{2}-1}=\left.C_{j_{2}} \quad\right|_{H_{j_{2}}} \quad \mathfrak{s}_{V} \supset E_{V}
$$


applying $\tau_{1}$, we get

$$
\tau_{1}\left(F_{V}^{\prime}\right), \tau_{1} C_{j_{2}-1}=\left.\tau_{1} C_{j_{2}} \quad\right|_{\tau_{1} H_{j_{2}}} \tau_{1} E_{V} .
$$

The gallery of chambers $\mathbf{c}^{1}$ is folded for the first time at the hyperplane $\tau_{1} H_{i_{2}}$, so $C_{V}^{-}$and $\tau_{1} C_{j_{2}-1}=\tau_{1} C_{j_{2}}$, and hence also $\tau_{1}\left(F_{V}^{\prime}\right)$ are on the same side of $\tau_{1} H_{i_{2}}$. Therefore, when we unfold with respect to $\tau_{1} H_{i_{2}}$, this wall separates $C_{V}^{-}$and $\tau_{1}\left(F_{V}^{\prime}\right)$ from $\tau_{2} \tau_{1}\left(F_{V}^{\prime}\right)$. This procedure can be iterated to show that at each step the image of $F_{V}^{\prime}$ is folded away from $C_{V}^{-}$, which proves that $(E \supset V \subset F)$ is positively folded.

Proof of Theorem 1;: " $\Rightarrow "$. Lemmas 5 and 6 show the existence of a minimal oneskeleton gallery $\left(E \supset V \subset E^{\prime}\right)$ such that $r_{-\infty}\left(E^{\prime}\right)=F$ implies that the one-skeleton gallery $(E \supset V \subset F)$ is positively folded.

Let $(E \supset V \subset F$ ) be a positively folded one-skeleton gallery. Let $\mathfrak{s} \subset \mathbb{A}$ be a sector with vertex $V$ containing $E$. Choose a chamber $D$ containing $F_{V}$ and let $w_{D}$ be the element that sends $C_{V}^{-}$to $D$. Let $\mathbf{i}=\left(i_{1}, \ldots, i_{r}\right)$ be the type of a reduced decomposition of $w_{D}=s_{i_{1}} \cdots s_{i_{r}}$ in $W_{V}^{v}$.

Lemma 7. For $w \leq w_{D}$ let $F_{V}^{\prime}$ be the a face of $w\left(C_{V}^{-}\right)$of the same type as $F_{V}$. Then there exists a gallery of chambers $\mathbf{c}=\left(C_{V}^{-}, C_{1}, \ldots, C_{r}\right)$ of type $\mathbf{i}$, positively folded with respect to $-w\left(C_{V}^{-}\right)$, such that $F_{V}^{\prime} \subset C_{r}$.

Proof. Let $\mathbf{m}$ be a minimal gallery of type $\mathbf{i}=\left(i_{1}, \ldots, i_{r}\right)$ between $C_{V}^{-}$and $D \supset F_{V}$. By the subword property, there exists a folded gallery $\mathbf{d}=\left(C_{V}^{-}, D_{1}, \ldots, D_{r}\right)$ of type $\mathbf{i}$ in $\mathbb{A}_{V}$ such that $D_{r} \supset F_{V}^{\prime}$.

Suppose the gallery is not positively folded with respect to $-w\left(C_{V}^{-}\right)$. Let $j$ be the smallest index such that $-w\left(C_{V}^{-}\right)$and $D_{j}=D_{j+1}$ are on the same side of the wall $H_{i_{j}}$ of type $i_{j}$.

The last chamber $D_{r}$ contains $F_{V}^{\prime} \subset w\left(C_{V}^{-}\right)$, and $w\left(C_{V}^{-}\right)$lies within the other half-space defined by $H_{i_{j}}$. It follows that the gallery $\mathbf{d}$ has to meet $H_{i_{j}}$ for some index larger than $j$. Let $j_{\text {max }}=\max _{k}\left\{H_{i_{k}}=H_{i_{j}}, k>j\right\}$ or set $j_{\max }=r$ if $H_{i_{j}} \supset F_{V}^{\prime}$.

Consider the new gallery of type $\mathbf{i}, \mathbf{d}^{\prime}=\left(C_{V}^{-}, D_{1}^{\prime}, \ldots, D_{r}^{\prime}\right)$ defined by :

$$
D_{k}^{\prime}= \begin{cases}D_{k} & \text { if } k \leqslant j \\ s_{H_{i_{j}}}\left(D_{k}\right) & \text { if } j+1 \leqslant k \leqslant j_{\max } \\ D_{k} & \text { if } k>j_{\max }\end{cases}
$$

This gallery still has the property that the last chamber contains $F_{V}^{\prime}: D_{r}^{\prime} \supset F_{V}^{\prime}$, and the gallery is now positively folded with respect to $-w\left(C_{V}^{-}\right)$till the index $i_{j}$. By repeating the procedure if necessary, one obtains a gallery $\mathbf{c}=\left(C_{V}^{-}, C_{1}, \ldots, C_{r}\right) \in \Gamma_{-w\left(C_{V}^{-}\right)}^{+}(\mathbf{i})$ such that $F_{V}^{\prime} \subset C_{r}$.

Lemma 8. Let $F_{V}^{\prime}$ be the face of $-\mathfrak{s}_{V}$ of the same type as $F_{V}$. Then there exists a face $E_{V}^{\prime}$ of the same type as $F_{V}$ such that $\left(E_{V}, E_{V}^{\prime}\right)$ is a minimal pair in $\mathcal{J}_{V}^{\mathfrak{a}}$ and $r_{C_{V}^{-}}\left(E_{V}^{\prime}\right)=F_{V}$.

Proof. Because $(E \supset V \subset F)$ is positively folded and $\mathfrak{s} \supset E$, the chamber $-\mathfrak{s}_{V}$ is closer to $C_{V}^{-}$than $D$. Therefore $w=w\left(C_{V}^{-},-\mathfrak{s}_{V}\right) \leq w_{D}$. So we can apply Lemma 7 to get a gallery of chambers $\mathbf{c}=\left(C_{V}^{-}, C_{1}, \ldots, C_{r}\right)$ of type $\mathbf{i}$ such that $\mathbf{c}$ is positively folded with respect to $\mathfrak{s}_{V}$ and $F_{V} \subset C_{r}$.

According to the preceding section (see Lemma 4 and before), there exist a minimal gallery $\mathbf{m}=\left(C_{V}^{-}, C_{1}^{\prime}, \ldots, C_{r}^{\prime}\right)$ in the cell $\mathcal{C}_{\mathfrak{s}_{V}}(\mathbf{c})$, and the chambers $C_{j}^{\prime}$ can be described as 
$C_{j}^{\prime}=g_{1} \cdots g_{j} C_{V}^{-}$where $g_{j}=c_{j}$ or $x_{c_{j}\left(\alpha_{i_{j}}\right)}\left(a_{j}\right) c_{j}$, and $c_{j} \neq 1$ if $w_{\mathfrak{s}_{V}}^{-1} \beta_{j}>0$, and $a_{j} \neq 0$ if $c_{j}=1$ and $w_{\mathfrak{s}_{V}}^{-1} \beta_{j}<0$.

Let $E_{V}^{\prime}$ be the face of the same type as $F_{V}^{\prime}$ contained in $C_{r}^{\prime}$. First, we note that the minimality of the gallery $\mathbf{m}=\left(C_{V}^{-}, C_{1}^{\prime}, \ldots, C_{r}^{\prime}\right)$ and the fact that $r_{C_{V}^{-}}(\mathbf{m})=\mathbf{c}$ ensures that $r_{C_{V}^{-}}\left(E_{V}^{\prime}\right)=F_{V}$. Second, we are going to prove that $\mathfrak{s}_{V}$ and $E_{V}^{\prime}$ are contained in the apartment $g \mathbb{A}_{V}$, with $g=g_{1} \cdots g_{r}$, and, in this apartment, $E_{V}^{\prime}$ is contained in the chamber opposite $\mathfrak{s}_{V}$.

The proof is by an inductive procedure. We show that, for all $j \in\{1, \ldots, r\}, \mathfrak{s}_{V}$ and $g_{1} \cdots g_{j} C_{V}^{-}$are in the apartment $g_{1} \cdots g_{j} \mathbb{A}_{V}$. We write in the following just $H_{j}$ for the common wall $H_{\beta_{j}}$ of $C_{j-1}$ and $C_{j}$ of type $i_{j}$.

By assumption, c is a positively folded gallery with respect to $\mathfrak{s}_{V}$, so there are three possible relative position for $\mathfrak{s}_{V}, C_{V}^{-}$and $C_{1}$ with respect to $H_{1}$ :

1) $\mathfrak{s}_{V}$ and $C_{V}^{-}$are on the same side of $H_{1}$ and $C_{1}$ not, then $C_{1}^{\prime}=g_{1} C_{V}^{-}=x_{-\alpha_{i_{1}}}\left(a_{1}\right) s_{i_{1}} C_{V}^{-}=$ $x_{-\alpha_{i_{1}}}\left(a_{1}\right) C_{1}$. But $x_{-\alpha_{i_{1}}}\left(a_{1}\right)$ pointwise stabilizes the halfspace bounded by $H_{1}$ containing $C_{V}^{-}$, hence $x_{-\alpha_{i_{1}}}\left(a_{1}\right)\left(\mathfrak{s}_{V}\right)=\mathfrak{s}_{V}$ and $C_{1}^{\prime}$ are in the apartment $g_{1} \mathbb{A}_{V}$;

2) $\mathfrak{s}_{V}$ and $C_{V}^{-}=C_{1}$ are separated by $H_{1}$, then $C_{1}^{\prime}=g_{1} C_{V}^{-}=x_{\alpha_{i_{1}}}\left(a_{1}\right) C_{V}^{-}$but $x_{\alpha_{i_{1}}}\left(a_{1}\right)$ pointwise stabilizes the halfspace bounded by $H_{1}$ not containing $C_{V}^{-}$, hence $\mathfrak{s}_{V}$ and $C_{1}^{\prime}$ are in the apartment $g_{1} \mathbb{A}_{V}$

3) $\mathfrak{s}_{V}$ and $C_{1}$ are on the same side of $H_{1}$ and $C_{V}^{-}$not, then $w_{\mathfrak{s}_{V}}$ has a reduced decomposition that starts with $s_{i_{1}}, w_{\mathfrak{s}_{V}}=s_{i_{1}} u$, so $w_{\mathfrak{s}_{V}}^{-1}\left(-\alpha_{i_{1}}\right)>0$, whence $g_{1}=c_{1}=s_{i_{1}}$ and $\mathfrak{s}_{V}$ and $C_{1}^{\prime}=s_{i_{1}} C_{V}^{-}$are in the apartment $g_{1} \mathbb{A}_{V}$.

By induction we assume now that the chambers $\mathfrak{s}_{V}$ and $g_{1} \cdots g_{j-1} C_{V}^{-}$are in the apartment $A_{j-1}=g_{1} \cdots g_{j-1} \mathbb{A}_{V}$. Again, we have three possible relative positions for $\mathfrak{s}_{V}, C_{j-1}$ and $C_{j}$ :

1) $\mathfrak{s}_{V}$ and $C_{j-1}$ are on the same side of $H_{j}$ and $C_{j}$ not, then $\mathfrak{s}_{V}$ and $C_{j-1}^{\prime}$ are on the same side of $g_{1} \cdots g_{j-1} H_{j}$ in $A_{j-1}$, and

$$
\begin{aligned}
C_{j}^{\prime} & =g_{1} \cdots g_{j-1} x_{-\alpha_{i_{j}}}\left(a_{j}\right) s_{i_{j}} C_{V}^{-} \\
& =g_{1} \cdots g_{j-1} x_{-\alpha_{i_{j}}}\left(a_{j}\right) s_{i_{j}}\left(g_{1} \cdots g_{j-1}\right)^{-1} C_{j-1}^{\prime} \\
& =g_{1} \cdots g_{j-1} x_{-\alpha_{i_{j}}}\left(a_{j}\right)\left(g_{1} \cdots g_{j-1}\right)^{-1} g_{1} \cdots g_{j-1} s_{i_{j}}\left(g_{1} \cdots g_{j-1}\right)^{-1} C_{j-1}^{\prime},
\end{aligned}
$$

where $g_{1} \cdots g_{j-1} s_{i_{j}}\left(g_{1} \cdots g_{j-1}\right)^{-1} C_{j-1}^{\prime}$ is the chamber adjacent to $C_{j}^{\prime}$ along $g_{1} \cdots g_{j-1} H_{j}$ in $A_{j-1}$. Moreover, $g_{1} \cdots g_{j-1} x_{-\alpha_{i_{j}}}\left(a_{j}\right)\left(g_{1} \cdots g_{j-1}\right)^{-1}$ pointwise stabilizes the halfspace bounded by $g_{1} \cdots g_{j-1} H_{j}$ containing $C_{j-1}^{\prime}$ and $\mathfrak{s}_{V}$. So $\mathfrak{s}_{V}$ and $C_{j}^{\prime}$ are in the apartment $g_{1} \cdots g_{j} \mathbb{A}_{V}$.

2) $C_{j-1}=C_{j}$ and $\mathfrak{s}_{V}$ are separated by $H_{j}$, then $C_{j-1}^{\prime}$ and $\mathfrak{s}_{V}$ are separated by $g_{1} \cdots g_{j-1} H_{j}$ in $A_{j-1}$, and $\mathfrak{s}_{V}$ and the chamber

$$
g_{1} \cdots g_{j-1} s_{i_{j}}\left(g_{1} \cdots g_{j-1}\right)^{-1} C_{j-1}^{\prime}
$$

are on the same side of this wall. Moreover, for $a_{j} \neq 0$

$$
C_{j}^{\prime}=g_{1} \cdots g_{j-1} x_{\alpha_{i_{j}}}\left(a_{j}\right) C_{V}^{-}=g_{1} \cdots g_{j-1} x_{\alpha_{i_{j}}}\left(a_{j}\right)\left(g_{1} \cdots g_{j-1}\right)^{-1} C_{j-1}^{\prime}
$$

is a chamber adjacent to $C_{j-1}^{\prime}$ along $g_{1} \cdots g_{j-1} H_{j}=g_{1} \cdots g_{j-1} x_{\alpha_{i_{j}}}\left(a_{j}\right) H_{j}$ in $g_{1} \cdots g_{j} \mathbb{A}_{V}$. The root-subgroup $g_{1} \cdots g_{j-1} x_{\alpha_{i_{j}}}\left(a_{j}\right)\left(g_{1} \cdots g_{j-1}\right)^{-1}$ pointwise stabilizes the halfspace bounded by $g_{1} \cdots g_{j-1} H_{j}$ and containing the chamber $g_{1} \cdots g_{j-1} s_{i_{j}}\left(g_{1} \cdots g_{j-1}\right)^{-1} C_{j-1}^{\prime}$. So $\mathfrak{s}_{V}$ and $C_{j}^{\prime}$ are in the apartment $g_{1} \cdots g_{j} \mathbb{A}_{V}$.

3) $\mathfrak{s}_{V}$ and $C_{j}$ are on the same side of $H_{j}$ and $C_{j-1}$ not, then $w_{\mathfrak{s}_{V}}^{-1} \beta_{j}>0$ and so $C_{j}^{\prime}=$ $g_{1} \cdots g_{j-1} s_{i_{j}} C_{V}^{-}$. Whence $\mathfrak{s}_{V}$ and $C_{j}^{\prime}$ are in the apartment $g_{1} \cdots g_{j} \mathbb{A}_{V}$. 
Therefore $\mathfrak{s}_{V}$ and $E_{V}^{\prime}$ are contained in the apartment $g \mathbb{A}_{V}=g_{1} \cdots g_{r} \mathbb{A}_{V}$, and in this apartment $E_{V}^{\prime}$ is the image of the face $F_{V}^{\prime}$ contained in $-\mathfrak{s}_{V}$. More precisely, $E_{V}^{\prime}=g \phi_{V}^{-}=$ $b w_{-\mathfrak{s}_{V}} \phi_{V}^{-}=b F_{V}^{\prime}$, where $\phi_{V}^{-}$is the face having the type of $F_{V}^{\prime}$ contained in $C_{V}^{-}, F_{V}^{\prime}=w_{-\mathfrak{s}_{V}} \phi_{V}^{-}$ and $b \in B_{\mathfrak{s}_{V}}=S t a b_{H_{V}}\left(\mathfrak{s}_{V}\right)$. This element is obtained as follows:

$$
\begin{aligned}
g & =g_{1} \cdots g_{r} \\
& =x_{c_{1}\left(\alpha_{i_{1}}\right)}\left(a_{1}\right) c_{1} \cdots x_{c_{r}\left(\alpha_{i_{r}}\right)}\left(a_{r}\right) c_{r} \\
& =x_{\beta_{1}}\left( \pm a_{1}\right) \cdots x_{\beta_{r}}\left( \pm a_{r}\right) c_{1} \cdots c_{r} \\
& =x_{\beta_{1}}\left( \pm a_{1}\right) \cdots x_{\beta_{r}}\left( \pm a_{r}\right) w_{-\mathfrak{s}_{V}} .
\end{aligned}
$$

As $E_{V}$ and $F_{V}^{\prime}$ are in opposite chambers in $\mathbb{A}_{V}$, so are $E_{V}$ and $E_{V}^{\prime}$ in $g \mathbb{A}_{V}$. Let $E^{\prime} \subset \mathcal{J}^{\mathfrak{a}}$ be the one dimensional face such that $V \subset E^{\prime}$ and $E_{V}^{\prime}$ is the associated face in the residue building $\mathcal{J}_{V}^{\mathfrak{a}}$. Let $r_{\infty, \mathfrak{s}}$ be the retraction from $\infty$, but now with respect to the sector $\mathfrak{s}$. On the level of the residue building, the retraction $r_{\infty, \mathfrak{s}}$ identifies with the retraction $r_{\mathfrak{s}_{V}}$ centered at $\mathfrak{s}_{V}$ of $\mathcal{J}_{V}^{\mathfrak{a}}$ onto $\mathbb{A}_{V}$. Since $E_{V}^{\prime}$ retracts with respect to $r_{\mathfrak{s}_{V}}$ onto $F_{V}^{\prime}$ in $\mathbb{A}_{V}, E^{\prime}$ retracts with respect to $r_{\infty, \mathfrak{s}}$ onto $F^{\prime}$ in $\mathbb{A}$. The retraction is distance preserving with respect to $\mathfrak{s}$, so the fact that $E$ and $F^{\prime}$ are in opposite sectors implies that the same holds for $E$ and $E^{\prime}$. In other words, $\left(E_{V}, E_{V}^{\prime}\right)$ is a minimal pair.

Proof of Theorem 1; " $\Leftarrow$ ". Since $(E \supset V \subset F)$ is positively folded, there exists a sector $\mathfrak{s} \supset E$ with vertex $V$ and a face $F^{\prime} \supset V$ of the same type as $F$ such that $F^{\prime} \subset-\mathfrak{s}$. Therefore, we can apply Lemmata 7 and 8 to get a minimal pair $\left(E_{V}, E_{V}^{\prime}\right)$, with $r_{C_{V}^{-}}\left(E_{V}^{\prime}\right)=F_{V}$, in other words a minimal gallery $\left(E \supset V \subset E^{\prime}\right)$, with $r_{-\infty}\left(E^{\prime}\right)=F$. The fact that $E^{\prime}$ has the same type as $F$ is a consequence of $r_{-\infty}\left(E^{\prime}\right)=F$.

Definition 14. Given a two-step gallery $(E \supset V \subset F)$ in $\mathcal{J}^{\mathfrak{a}}$, denote by $\operatorname{Min}(E, F)$ the set of all faces $E^{\prime} \supset V$ such that $r_{-\infty}\left(E^{\prime}\right)=F$ and $\left(E \supset V \subset E^{\prime}\right)$ is minimal. This set can be identified with the set of all faces $E_{V}^{\prime}$ such that $r_{C_{V}^{-}}\left(E_{V}^{\prime}\right)=F_{V}$ and $\left(E_{V}^{\prime}, E_{V}\right)$ is a minimal pair.

We assume now that ( $E \supset V \subset F$ ) is positively folded, we want to give this set an algebraic structure as an open subset of a union of cells in a Bott-Samelson variety.

We use the same notation as in section 6.1. Let $\mathfrak{s}$ be a sector containing $E$ and let $w_{\mathfrak{s}_{V}}=w\left(C_{V}^{-}, \mathfrak{s}_{V}\right)$ be the element in $W_{V}^{v}$ that sends $C_{V}^{-}$to $\mathfrak{s}_{V}$. Let $D$ be the chamber containing $F_{V}$ the closest to $C_{V}^{-}$. Since $(E \supset V \subset F)$ is positively folded, $w_{-\mathfrak{s}_{V}}=w\left(C_{V}^{-},-\mathfrak{s}_{V}\right) \leq w_{D}=$ $w\left(C_{V}^{-}, D\right)$. Fix a reduced decomposition of $w_{D}=s_{i_{1}} \cdots s_{i_{r}}$ in $W_{V}^{v}$ and denote its type by $\mathbf{i}=\left(i_{1}, \ldots, i_{r}\right)$.

We denote by $\Gamma_{\mathfrak{S}_{V}}^{+}(\mathbf{i}, o p)$ the set of all galleries $\mathbf{c}=\left(C_{V}^{-}, C_{1}, \ldots, C_{r}\right)$ of residue chambers of type $\mathbf{i}$ which are positively folded with respect to $\mathfrak{s}_{V}$ and have the property that the face $F_{V}^{\prime}$ of the same type as $F_{V}$ contained in $C_{r}$ forms a minimal pair with $E_{V}$ in $\mathbb{A}_{V}$.

Proposition 13. The set $\operatorname{Min}(E, F)$ is in bijection with the disjoint union $\coprod_{\mathbf{c} \in \Gamma_{\mathfrak{s}_{V}}^{+}(\mathbf{i}, \text { op })} \mathcal{C}_{\mathfrak{s}_{V}}^{m}(\mathbf{c})$, where $\mathcal{C}_{\mathfrak{s}_{V}}^{m}(\mathbf{c})$ is the set of all minimal galleries in the cell $\mathcal{C}_{\mathfrak{s}_{V}}(\mathbf{c}) \subset \mathrm{BS}(\mathbf{i})$.

Proof. First recall that $\operatorname{Min}(E, F)$ identifies with the set of all faces $E_{V}^{\prime}$ such that $\left(E_{V}, E_{V}^{\prime}\right)$ is a minimal pair and $r_{C_{V}^{-}}\left(E_{V}^{\prime}\right)=F_{V}$. Next, the proof of Lemma 8 asserts that to a minimal gallery $\mathbf{m}^{\prime} \in \mathcal{C}_{\mathfrak{s}_{V}}(\mathbf{c})$ corresponds such a unique face $E_{V}^{\prime}=g\left(\mathbf{m}^{\prime}\right)$. It is the face of the same type as $F_{V}$ contained in the last chamber of $\mathbf{m}^{\prime}$. Lemma $[5$ shows that this mapping $g$ is surjective. 
Suppose now that $\mathbf{m}^{\prime}, \mathbf{n}^{\prime} \in \mathcal{C}_{\mathfrak{s}_{V}}(\mathbf{c})$ are two minimal galleries such that $E_{V}^{\prime}=g\left(\mathbf{m}^{\prime}\right)=g\left(\mathbf{n}^{\prime}\right)$. Since $D$ is the closest chamber to $C_{V}^{-}$containing $F_{V}$ the last chambers of $\mathbf{m}^{\prime}$ and of $\mathbf{n}^{\prime}$ have to be the same. Since they have the same type and the same origin, $\mathbf{m}^{\prime}=\mathbf{n}^{\prime}$.

\section{From local properties to global properties}

In Theorem 1 we have shown that one can obtain a minimal two steps gallery by "unfolding" a combinatorial two steps gallery $(E \supset V \subset F)$ only if the latter is positively folded. This provides a procedure to unfold a locally positively folded combinatorial one-skeleton gallery inductively to get a locally minimal one-skeleton gallery. The first aim of this section is to show that if one starts with a globally positively folded gallery, then this unfolding algorithm produces automatically globally minimal one-skeleton galleries. Next we derive a formula for the polynomials $L_{\lambda, \mu}$.

\subsection{From positively folded two-steps galleries to minimal galleries}

Proposition 14. Let $\delta=\left[\delta_{0}, \delta_{1}, \ldots, \delta_{r}\right]=\left(V_{0} \subset E_{0} \supset \cdots \subset E_{r} \supset V_{r+1}\right) \in \Gamma\left(\gamma_{\lambda}\right)$. The intersection $\{$ minimal galleries $\} \cap C_{\delta}$ is non-empty if, and only if, $\delta$ is positively folded.

Proof. Let $\gamma=\left(V_{0} \subset E_{0}^{\prime} \supset V_{1}^{\prime} \subset \cdots \supset V_{r}^{\prime} \subset E_{r}^{\prime} \supset V_{r+1}^{\prime}\right)$ be a minimal one-skeleton gallery in the cell $C_{\delta}$. Since $\gamma$ starts at $V_{0}=\mathfrak{o}$, we may replace $\gamma$ by $u \gamma$ for some $u \in U^{-}(\mathcal{O})$ if necessary and assume that $E_{0}^{\prime}=E_{0}$ and $V_{1}^{\prime}=V_{1}$ are in $\mathbb{A}$. Let $\underline{\mathfrak{s}}^{\prime}(\gamma)=\left(\mathfrak{s}_{0}, \mathfrak{s}_{1}^{\prime}, \ldots, \mathfrak{s}_{r}^{\prime}\right)$ be the sequence of representatives of the same equivalence class of sectors such that $V_{i}^{\prime}$ is the vertex of $\mathfrak{s}_{i}^{\prime}$ and $E_{i}^{\prime} \subset \mathfrak{s}_{i}^{\prime}$. The sequence starts with a sector tipped at 0 whose image by $r_{-\infty}$ is the chamber $\tau_{0}\left(C^{+}\right)$, for some $\tau_{0} \in W$. We know that $\left(E_{0} \supset V_{1} \subset E_{1}^{\prime}\right)$ is minimal and such that $r_{-\infty}\left(E_{1}^{\prime}\right)=E_{1}$, hence Lemma $\left[6\right.$ shows that $\left(E_{0} \supset V_{1} \subset E_{1}\right)$ is positively folded. This means that there exists a face $V_{1} \subset E_{1}^{\prime \prime} \subset \mathbb{A}$ such that $\left(E_{0} \supset V_{1} \subset E_{1}^{\prime \prime}\right)$ is minimal and $\left(E_{0} \supset V_{1} \subset E_{1}\right)$ is obtained from $\left(E_{0} \supset V_{1} \subset E_{1}^{\prime \prime}\right)$ by a positive folding, see Lemma 5 and Lemma 6 and the proofs. Now in the proof one may choose for the minimal gallery of residue chambers as last chamber the residue chamber associated to $\mathfrak{s}_{0}\left(V_{1}\right)=\mathfrak{s}_{1}$. But $E_{1}^{\prime \prime}$ is contained in $\tau_{0}\left(C^{+}\right)\left(V_{1}\right)$ (see Lemma 5 and its proof) and the sector $\mathfrak{s}_{1}$ retracts onto a sector of $\mathbb{A}$ tipped at $V_{1}$ and containing $E_{1}$. Therefore $r_{-\infty}\left(\mathfrak{s}_{1}\right)=\tau_{1}\left(C^{+}\right)\left(V_{1}\right)$, with $\tau_{0} \geq \tau_{1}$.

We want to repeat this argument to prove the claim in an inductive procedure. To do so, recall from Proposition 6 , that $\gamma$ corresponds to a sequence

$$
\left(v_{0}, v_{1}, \ldots, v_{r}\right) \in \mathrm{Stab}_{-}\left(V_{0}, E_{0}\right) \times \mathrm{Stab}_{-}\left(V_{1}, E_{1}\right) \times \cdots \times \mathrm{Stab}_{-}\left(V_{r}, E_{r}\right)
$$

and that $E_{j}^{\prime}=v_{0} v_{1} \cdots v_{j} \delta_{0} \delta_{1} \cdots \delta_{j} E_{j}^{f}=v_{0} v_{1} \cdots v_{j} E_{j}$. The gallery $\gamma$ can be retracted step by step, that means that we consider the sequence:

$$
\begin{aligned}
\gamma= & \left(V_{0} \subset E_{0}^{\prime} \supset V_{1}^{\prime} \subset \cdots \supset V_{r}^{\prime} \subset E_{r}^{\prime} \supset V_{r+1}^{\prime}\right), \\
\gamma^{0}= & \left(V_{0} \subset E_{0} \supset V_{1} \subset v_{1} E_{1} \cdots \subset\left(v_{1} \cdots v_{r}\right) E_{r} \supset\left(v_{1} \cdots v_{r}\right) V_{r+1}\right), \\
\vdots & \\
\gamma^{j-1}= & \left(V_{0} \subset E_{0} \supset V_{1} \subset \cdots \subset E_{j-1} \supset V_{j} \subset v_{j} E_{j} \supset \cdots\right. \\
& \left.\cdots \subset\left(v_{j} \cdots v_{r}\right) E_{r} \supset\left(v_{j} \cdots v_{r}\right) V_{r+1}\right) \\
\vdots & \\
\gamma^{r}=\delta= & \left(V_{0} \subset E_{0} \supset V_{1} \subset \cdots \supset V_{r} \subset E_{r} \supset V_{r+1}\right) .
\end{aligned}
$$


Now, at each step, $\left(E_{j-1} \supset V_{j} \subset v_{j} E_{j}\right)$ is minimal because it is obtained from a minimal two-step gallery by applying elements of $G(\mathcal{K})$. So, we can repeat the previous arguments to show that $\delta$ is globally positively folded.

Reciprocally, we show that if $\delta$ is positively folded then one can, inductively, built a minimal gallery that retracts onto it. Indeed, we start applying Theorem 1 at the vertex $V_{r}$. So we get a gallery

$$
\delta^{r}=\left(V_{0} \subset E_{0} \supset V_{1} \subset \cdots \subset E_{r-1} \supset V_{r} \subset E_{r}^{\prime} \supset V_{r+1}^{\prime}\right),
$$

where $\left(E_{r-1} \supset V_{r} \subset E_{r}^{\prime}\right)$ is minimal in an apartment $A_{r}, r_{-\infty}\left(E_{r}^{\prime}\right)=E_{r}$ and a sequence of sectors $\left(\mathfrak{s}_{0}, \mathfrak{s}_{1}, \ldots, \mathfrak{s}_{r}\right)$ such that $\underline{\mathfrak{s}}_{0} \geq \mathfrak{s}_{1} \geq \cdots \geq \underline{\mathfrak{s}}_{r-1}=\underline{\mathfrak{s}}_{r}$. We apply the theorem again at the vertex $V_{r-1}$. So we get a gallery

$$
\delta^{r-1}=\left(V_{0} \subset E_{0} \supset V_{1} \subset \cdots \subset E_{r-2} \supset V_{r-1} \subset E_{r-1}^{\prime} \supset V_{r}^{\prime}\right),
$$

where $\left(E_{r-2} \supset V_{r-1} \subset E_{r-1}^{\prime}\right)$ is minimal in an apartment $A_{r-1}, r_{-\infty}\left(E_{r-1}^{\prime}\right)=E_{r-1}$ and a sequence of sectors $\left(\mathfrak{s}_{0}, \mathfrak{s}_{1}, \ldots, \mathfrak{s}_{r-1}\right)$ such that $\underline{\mathfrak{s}}_{0} \geq \mathfrak{s}_{1} \geq \cdots \geq \underline{\mathfrak{s}}_{r-2}=\underline{\mathfrak{s}}_{r-1}$. Now, since $\left(E_{r-1} \supset V_{r} \subset E_{r}^{\prime}\right)$ is positively folded in $\mathbb{A}$, there exists a face $F_{r}^{\prime}$ of the same type as $E_{r}$ such that $\left(E_{r-1} \supset V_{r} \subset F_{r}^{\prime}\right)$ is minimal in $\mathbb{A}$. Since $E_{r-1}^{\prime}=u_{r-1} E_{r-1}$, we can take $A_{r-1}=u_{r-1} \mathbb{A}$ and the image of $\left(E_{r-1} \supset V_{r} \subset F_{r}^{\prime}\right)$ in $A_{r-1}$ is still minimal. So we complete the gallery $\delta^{r-1}$ with it to get a one-skeleton gallery which is minimal after the index $r-1$ and contained in the sector $\mathfrak{s}_{r-2}=\mathfrak{s}_{r-1}=\mathfrak{s}_{r}$ of $A_{r-1}$. Iterating this procedure, we get a minimal one-skeleton gallery that retracts onto $\delta$.

\subsection{A formula for $L_{\lambda, \mu}$}

For a dominant coweight $\lambda$ let $\gamma_{\lambda}$ be a dominant combinatorial gallery joining $\mathfrak{o}$ and $\lambda$ (see Example (4). The investigation of the intersection $Z_{\lambda, \mu}$ can be transferred to the Bott-Samelson variety $\Sigma\left(\gamma_{\lambda}\right)$ :

$$
Z_{\lambda, \mu}=G(\mathcal{O}) \cdot \lambda \cap U^{-}(\mathcal{K}) \cdot \mu=\bigcup_{\substack{\delta \in \Gamma\left(t_{\gamma_{\lambda}}, \mathfrak{o}\right) \\ \operatorname{target}(\delta)=\mu}}\{\text { minimal galleries }\} \cap C_{\delta}
$$

Proposition 14 states that the intersection \{minimal galleries $\} \cap C_{\delta}$ is non-empty if and only if $\delta$ is positively folded. We want to describe the intersection more precisely.

Recall from Definition 14 that for a two steps gallery $(E \supset V \subset F)$ the set $\operatorname{Min}(E, F)$ identifies with the set of all faces $E_{V}^{\prime}$ such that $\left(E_{V}, E_{V}^{\prime}\right)$ is a minimal pair and $r_{C_{V}^{-}}\left(E_{V}^{\prime}\right)=F_{V}$.

Let $\delta=\left(\mathfrak{o}=V_{0} \subset E_{0} \supset \cdots \supset V_{r} \subset E_{r} \supset \mu\right)$ be a positively folded combinatorial gallery and let $B^{-} \subset G$ be the opposite Borel subgroup. Denote by $D_{0}$ the chamber in $\mathbb{A}$ which contains $E_{0}$ and is the closest to $C^{-}$, and let $w_{D_{0}} \in W$ be the element such that $w_{D_{0}}\left(C^{-}\right)=D_{0}$.

Proposition 15. The set of all minimal one-skeleton galleries in the cell $C_{\delta}$ identifies with the product

$$
B^{-} w_{D_{0}} Q_{E_{0}}^{-} / Q_{E_{0}}^{-} \times \prod_{j=1}^{r} \operatorname{Min}\left(E_{j-1}, E_{j}\right) .
$$


Proof. If $\gamma=\left(V_{0} \subset E_{0}^{\prime} \supset V_{1}^{\prime} \subset \cdots \supset V_{r}^{\prime} \subset E_{r}^{\prime} \supset V_{r+1}^{\prime}\right)$ is a minimal gallery of $C_{\delta}$, then $E_{0}^{\prime}$ identifies with an element of the orbit $B^{-} w_{D_{0}} Q_{E_{0}}^{-} / Q_{E_{0}}^{-}$. Further, to $\gamma \in C_{\delta}$ corresponds a sequence $\left(v_{0}, v_{1}, \ldots, v_{r}\right)$ in

$$
\operatorname{Stab}_{-}(\delta)=\operatorname{Stab}_{-}\left(V_{0}, E_{0}\right) \times \operatorname{Stab}_{-}\left(V_{1}, E_{1}\right) \times \cdots \times \operatorname{Stab}_{-}\left(V_{r}, E_{r}\right) .
$$

In the proof of Proposition 14, we have seen that $h(\gamma):=\left(E_{0}^{\prime}, v_{1} E_{1}, \ldots, v_{r} E_{r}\right)$ belongs to $B^{-} w_{D_{0}} Q_{E_{0}}^{-} / Q_{E_{0}}^{-} \times \prod_{j=1}^{r} \operatorname{Min}\left(E_{j-1}, E_{j}\right)$, and we have also seen that $h$ is surjective. The fact that $h$ is injective is a consequence of Proposition 4 .

Let $\mathbb{F}_{q}$ be the finite field with $q$ elements and replace the field of complex numbers by the algebraic closure $K$ of $\mathbb{F}_{q}$. Assume that all groups are defined and split over $\mathbb{F}_{q}$. We replace now $\mathcal{K}$ by $\mathcal{K}_{q}=\mathbb{F}_{q}((t))$, the field of Laurent series, and $\mathcal{O}$ by $\mathcal{O}_{q}=\mathbb{F}_{q}[[t]]$. For a given positively folded gallery $\delta=\left[\delta_{0}, \delta_{1}, \ldots, \delta_{r}\right]=\left(V_{0} \subset E_{0} \supset V_{1} \subset \cdots \subset E_{r} \supset V_{r+1}\right)$ we want to count the number of points (over $\mathbb{F}_{q}$ ) of the intersection

$$
\{\text { minimal galleries }\} \cap C_{\delta} \text {. }
$$

For convenience, we first fix (and recall) some notation: $\forall j=0,1, \ldots, r$, let

- $D_{j}$ be the closest chamber to $C_{V_{j}}^{-}$containing $\left(E_{j}\right)_{V_{j}}$;

- $\mathfrak{s}^{j} \supset E_{j-1}$ be a sector with vertex $V_{j}$ such that there exists a face $F_{j}^{\prime} \subset-\mathfrak{s}^{j}$ containing $V_{j}$ of the same type as $E_{j}$;

- $\mathbf{i}_{j}=\left(\left(i_{j}\right)_{1}, \ldots,\left(i_{j}\right)_{r_{j}}\right)$ be a reduced decomposition of $w\left(C_{V_{j}}^{-}, D_{j}\right)$.

We denote by $\Gamma_{\mathfrak{s}_{V_{j}}^{j}}^{+}\left(\mathbf{i}_{j}, o p\right)$ the set of all galleries $\mathbf{c}=\left(C_{V_{j}}^{-}, C_{1}, \ldots, C_{r_{j}}\right)$ of residue chambers of type $\mathbf{i}_{j}$ which are positively folded with respect to $\mathfrak{s}_{V_{j}}^{j}$ and have the property that the face $\left(E_{j}^{\prime}\right)_{V_{j}}$ of the same type as $\left(E_{j}\right)_{V_{j}}$ contained in $C_{r_{j}}$ forms a minimal pair with $\left(E_{j-1}\right)_{V_{j}}$ in $\mathbb{A}_{V_{j}}$.

The exponents in the formula are, first, the length $\ell\left(w_{D_{0}}\right)$ and, second, for each $\mathbf{c}=$ $\left(c_{1}, \ldots, c_{r_{j}}\right) \in \Gamma_{\mathfrak{s}_{V_{j}}^{j}}^{+}\left(\mathbf{i}_{j}, o p\right)$, the nonnegative integers $t(\mathbf{c})$ and $r(\mathbf{c})$ defined in Lemma 4; $t(\mathbf{c})=$ $\sharp\left\{k \mid c_{k}=s_{\left(i_{j}\right)_{k}}\right.$ and $\left.w_{\mathfrak{s}_{V_{j}}^{j}}^{-1} \beta_{k}<0\right\}$ and $r(\mathbf{c})=\sharp\left\{j \mid c_{k}=1\right.$ and $\left.w_{\mathfrak{s}_{V_{j}}^{j}}^{-1} \beta_{k}<0\right\}$. Combining Lemma 4, Theorem 1 and Propositions 13-15, we obtain the following formula:

\section{Theorem 2.}

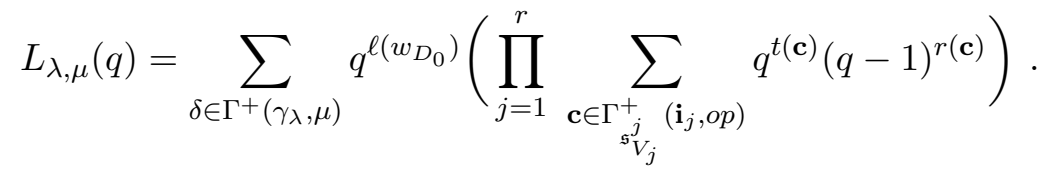

Remark 7. According to a result of Katz (Theorem 6.1.12) in [11]), the value $L_{\lambda, \mu}(1)$ gives the Euler-Poincaré characteristic of the variety $G(\mathcal{O}) . \lambda \cap U^{-}(\mathcal{K}) . \mu$. Now a summand above is nonzero if and only if the gallery is minimal. It is easy to see that a gallery is minimal if and only if the gallery has as a target an extremal weight. Thus, we recover a result of Ngô and Polo [24, saying that this characteristic is 1 if $\mu$ is in the orbit $W \lambda$, and it is 0 otherwise. 
Example 9. Let us consider an example in type $A_{2}$. Let $\lambda=2 \omega_{1}+\omega_{2}$ where $\omega_{i}$ are the fundamental coweights. There are three possibilities for $\mu \leq \lambda$ in the fundamental Weyl chamber: $\mu=\lambda, \mu=2 \omega_{2}$ and $\mu=\omega_{1}$. If $\mu=\lambda$, then one finds $L_{\lambda, \lambda}(q)=q^{2} q^{2} q^{2}$. In the second case, $L_{\lambda, 2 \omega_{2}}(q)=q(q-1) q q^{2}$. Finally, if $\mu=\omega_{1}$, there are two one-skeleton galleries starting in $\mathfrak{o}$ and ending in $\omega_{1}$. Let us explain the computation in the case of the gallery $\left(\mathfrak{o} \subset E_{1} \supset V_{1} \subset E_{2} \supset V_{2} \subset E_{3} \supset \omega_{1}\right)$ plotted in the picture below.

At the vertex $\mathfrak{o}, \ell\left(w_{D_{0}}\right)=1$, therefore we get a $q$. At the vertex $V_{1}$, there is only one gallery $\mathbf{c}$ of residue chambers positively folded with respect to $\mathfrak{s}^{1}$, starting in $C_{V_{1}}^{-}$and ending in a chamber containing an opposite to $E_{1}$. This gallery $\mathbf{c}$ has a positively (with respect to $\mathfrak{s}^{1}$ ) folding on one wall and crosses positively another, so we have $t(\mathbf{c})=1$ and $r(\mathbf{c})=1$, whence we get $(q-1) q$. At the vertex $V_{2}$, the gallery $\left(E_{2} \supset V_{2} \subset E_{3}\right)$ is minimal. The gallery of residue chambers has only two terms and positively (with respect to another sector) crosses the vertical wall, therefore, we get $q$. One computes in an analogous way the number of minimal one-skeleton galleries retracting on the second gallery ending in $\omega_{1}$ and one gets $q(q-1) q^{2}$. Finally, $L_{\lambda, \omega_{1}}(q)=q(q-1) q q+q(q-1) q^{2}=2(q-1) q^{3}$.

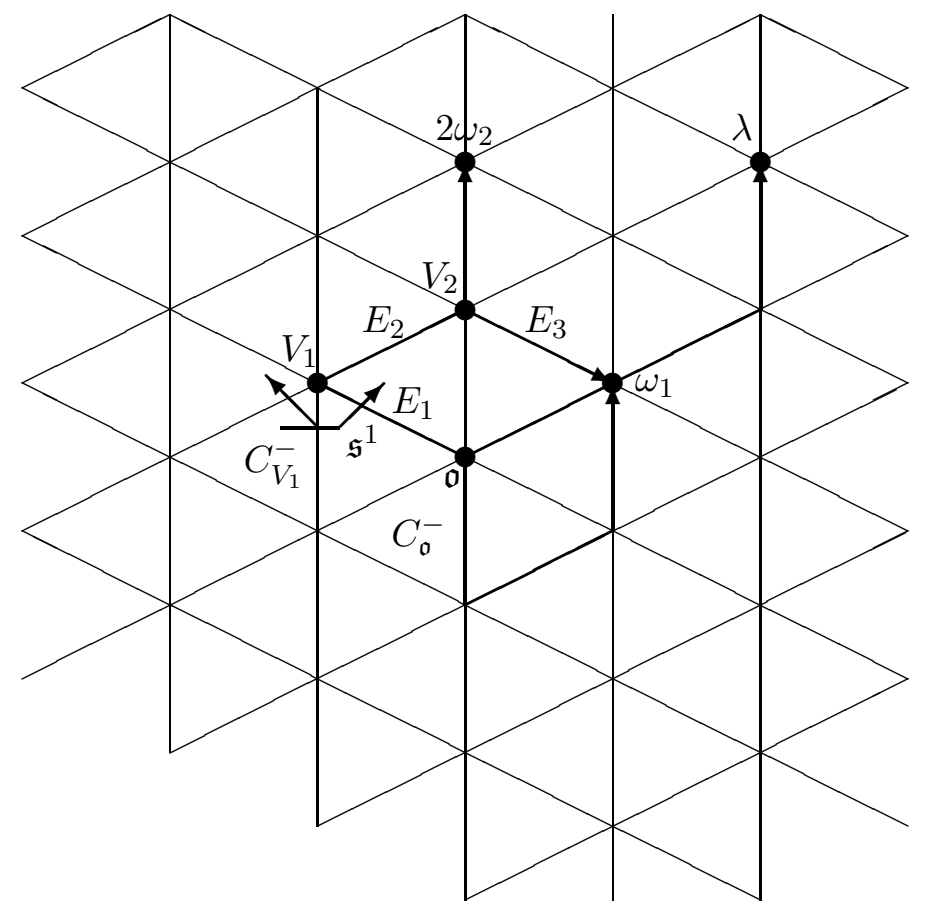

\section{Dimension of $r_{-\infty}^{\min }(\delta)$, LS-galleries and Young tableaux}

We want now to discuss some examples and the connection with the work of Lakshmibai, Musili and Seshadri. Recall that the theory of a path model for a representation is a generalization of the original idea of Lakshmibai, Musili and Seshadri (see for example [14, [16, [17]) to index a basis of fundamental representation by sequences of Weyl group elements satisfying certain combinatorial conditions. Monomials of these basis elements then form a 
generating system for the other irreducible representations (respectively the corresponding dual Weyl modules in positive characteristic), and the aim was to show that special monomials, the standard monomials = monomials having a defining chain, form in fact a basis. This program was successfully realized in many cases, for example for all representations of the classical groups but also in many other cases (see ibidem). The path model theory provided a new approach and made it possible to prove the conjecture for the character in full generality for Kac-Moody algebras [20], the construction of an associated standard monomial theory is discussed in [22].

\subsection{LS one-skeleton galleries}

Given a dominant coweight $\lambda$, let $\gamma_{\lambda}$ be a combinatorial one-skeleton gallery as in Example 4. Let $\delta=\left(V_{0}=\mathfrak{o} \subset E_{0} \supset \ldots \supset V_{r+1}\right)$ be a positively folded combinatorial one-skeleton gallery of the same type as $\gamma_{\lambda}$. By Proposition 14 we know that the intersection of the set of minimal galleries $G(\mathcal{O}) \cdot \gamma_{\lambda}$ with the cell $C_{\delta}$ is a dense subset of $C_{\delta}$, so

$$
\operatorname{dim} r_{-\infty}^{\min }(\delta)=\operatorname{dim}\left(\{\text { minimal galleries }\} \cap C_{\delta}\right)=\operatorname{dim}\left(C_{\delta}\right) .
$$

The dimension of the cell can be computed by Proposition 6 using combinatorial properties of the gallery: given an affine root $(\alpha, n), \alpha>0$, a vertex $V \in H_{\alpha, n}$ and an edge $E$ in $\mathbb{A}$, then we say that $(V, E)$ crosses the wall (or hyperplane) $H_{\alpha, n}$ in the positive (negative) direction if $F \not \subset H_{\alpha, n}^{-}$(respectively $\left.F \not \subset H_{\alpha, n}^{+}\right)$.

Remark 8. Using the terminology of section 4.4, an equivalent formulation is to say that a wall crossing is positive if $(-\alpha,-n) \in \Phi_{-}^{\mathfrak{a}}(V, F)$.

For the gallery $\delta$ denote by $\sharp^{+} \delta$ the number of positive wall crossings, by $\sharp^{-} \delta$ the number of negative wall crossings and by $\sharp^{ \pm} \delta$ the number of all wall crossings:

$$
\begin{aligned}
& \sharp^{+} \delta=\sum_{i=0}^{r}\left(\sharp \text { positive wall crossings of }\left(V_{i}, E_{i}\right)\right) \\
& \sharp^{-} \delta=\sum_{i=0}^{r}\left(\sharp \text { negative wall crossings of }\left(V_{i}, E_{i}\right)\right) \\
& \sharp^{ \pm} \delta=\sharp^{+} \delta+\sharp^{-} \delta .
\end{aligned}
$$

For the last number we have $\sharp^{ \pm} \delta=\sharp^{+} \gamma_{\lambda}=\langle\lambda, 2 \rho\rangle$ because it depends only on the type of the gallery. Together with Remark 8 and Proposition 6 we get:

Lemma 9. $\sharp^{+} \delta=\operatorname{dim}\left(C_{\delta}\right)$.

An upper bound for $\sharp^{+} \delta$ can be determined using the target of the gallery:

Proposition 16. Let $\mu$ be the target of $\delta$, then $\sharp^{+} \delta \leq\langle\lambda+\mu, \rho\rangle$.

Proof. Since $P_{\lambda} \rightarrow s_{\lambda}$ we know that $q^{-\langle\rho, \lambda+\mu\rangle} L_{\lambda, \mu} \in \mathbb{Z}\left[q^{-1}\right]$, so the power of the leading term in $L_{\lambda, \mu}$ is less or equal to $\langle\rho, \lambda+\mu\rangle$. By the formula in Theorem 2 , the maximal power of the contribution coming from a positively folded gallery $\delta$ occurs with coefficient +1 . The maximal power of the term coming from $\delta$ is $\operatorname{dim}\left(C_{\delta}\right)$, which proves the claim.

Definition 15. We call a positively folded combinatorial one-skeleton gallery $\delta$ of the same type as $\gamma_{\lambda}$ a $L S$-gallery if $\sharp^{+} \delta=\langle\lambda+\mu, \rho\rangle$, where $\mu$ is the target of $\delta$. 
Remark 9. All minimal combinatorial one-skeleton galleries are LS-galleries. Indeed, if $\delta$ is a minimal gallery with target $\mu$, then $\langle\mu, 2 \rho\rangle=\sharp^{+} \delta-\sharp^{-} \delta$, so

$$
\sharp^{+} \delta=\frac{1}{2}\left(\sharp^{+} \delta-\sharp^{-} \delta+\sharp^{ \pm} \delta\right)=\frac{1}{2}\langle\lambda+\mu, 2 \rho\rangle=\langle\lambda+\mu, \rho\rangle .
$$

\subsection{Reduction to the case of a fundamental weight}

To describe the connection of the path model with LS-galleries in the one-skeleton, one of the first steps is the reduction to the case of a fundamental weight.

Lemma 10. 1. If $\omega$ is a minuscule coweight, then all combinatorial galleries of the same type as $\gamma_{\omega}$ are LS-galleries.

2. Suppose $\delta_{1}, \ldots, \delta_{r}$ are positively folded combinatorial galleries of the same type as $\gamma_{\lambda_{1}}, \ldots, \gamma_{\lambda_{r}}$ respectively. Suppose the concatenation $\delta=\delta_{1} * \ldots * \delta_{r}$ is positively folded. Then $\delta$ is an LS-gallery if and only if each of the $\delta_{j}, j=1, \ldots, r$, is a LS-gallery.

Proof. If $\omega$ is a minuscule coweight, then all combinatorial galleries of the same type as $\gamma_{\omega}$ have no folds and hence are minimal, which proves the claim by Remark 9 ,

Let $\delta=\delta_{1} * \ldots * \delta_{r}$ be a concatenation of positively folded galleries as in (3). If $\delta$ has target $\mu$ and $\delta_{i}$ has target $\mu_{i}$, then $\sharp^{+} \delta=\sum_{j=1}^{r} \sharp^{+} \delta_{j}$ and $\langle\lambda+\mu, \rho\rangle=\sum_{j=1}^{r}\left\langle\lambda_{j}+\mu_{j}, \rho\right\rangle$. So by Proposition [16] we have equality $\sharp^{+} \delta=\langle\lambda+\mu, \rho\rangle$ if and only if $\sharp^{+} \delta_{j}=\left\langle\lambda_{j}+\mu_{j}, \rho\right\rangle$ for all $j=1, \ldots, r$.

In the following let $\gamma_{\lambda}$ be as in Example 2, we want to characterize the LS-galleries of the same type as $\gamma_{\lambda}$. The Lemma above reduces the consideration to the case where $\lambda=\omega$ is a fundamental weight.

Let $\delta_{0}=\left(\mathfrak{o}=V_{0} \subset E_{0} \supset \ldots \subset V_{j} \subset \ldots \supset V_{r}=\mu_{0}\right)$ be a positively folded gallery of the same type as $\gamma_{\omega}$, and let $j$ be such that $\delta_{0}$ has no folds at the vertices $V_{i}$ for $i \geqslant j$ (note: we do not ask $j$ to be minimal with this property). Let $\beta$ be a positive root and suppose there exists an $m \in \mathbb{Z}$ such that $V_{j} \in H_{\beta, m}$. Denote by $\delta$ the gallery

$$
\delta=\left(\mathfrak{o}=V_{0} \subset E_{0} \supset \ldots \subset V_{j} \subset s_{\beta, m}\left(E_{j}\right) \supset \ldots \supset s_{\beta, m}\left(V_{r}\right)=\mu\right)
$$

Given the one-dimensional face $E_{j}$ let $\nu_{E_{j}}$ be the rational weight $V_{j+1}-V_{j}$. Since $E_{j}$ is of type $\omega$, there exists a unique element $\tau_{E_{j}} \in W / W_{\omega}$ such that the two rays $\mathbb{R} \tau_{E_{j}}(\omega)$ and $\mathbb{R} \nu_{E_{j}}$ coincide.

Definition 16. We say that $\delta$ is obtained from $\delta_{0}$ by a positive fold if $s_{\beta} \tau_{E_{j}}<\tau_{E_{j}}$ in the Bruhat order on $W / W_{\omega}$. We say that $\delta$ is obtained from $\delta_{0}$ by an $L S$-fold if in addition $\ell\left(s_{\beta} \tau_{E_{j}}\right)=\ell\left(\tau_{E_{j}}\right)-1$ for the length function $\ell$ on $W / W_{\omega}$

By definition, if $\delta$ is obtained from $\delta_{0}$ by a positive fold, then $\delta$ is also positively folded. Obviously every positively folded gallery can be obtained from a minimal gallery by a sequence of such positive folds.

To be able to characterize the LS-galleries of the same type as $\gamma_{\omega}$, we divide this folding algorithm into the smallest possible steps. Since we can only fold with respect to the roots in the local root system $\Phi_{V_{j}}$, we consider first the Weyl group $W_{V_{j}}$ of $\Phi_{V_{j}}$. There exists a unique ray $\mathbb{R} \nu_{0}$ contained in the dominant Weyl chamber with respect to $\Phi_{V_{j}}$ and a unique element $t \in W_{V_{j}} /\left(W_{V_{j}}\right)_{\nu_{0}}$ such that $t\left(\nu_{0}\right)=\nu_{E_{j}}$. 
Definition 17. We say that the fold by $s_{\beta, m}$ is minimal for the local root system $\Phi_{V_{j}}$ if $\ell\left(s_{\beta} t\right)=\ell(t)-1$ for the length function $\ell$ on $W_{V_{j}} /\left(W_{V_{j}}\right)_{\nu_{0}}$.

If the fold is not minimal, then one can find positive roots $\beta_{1}, \ldots, \beta_{q}$ in $\Phi_{V_{j}}$ such that $t>s_{\beta_{1}} t>\ldots>s_{\beta_{q}} \cdots s_{\beta_{1}} t=s_{\beta} t$ in the Bruhat ordering on $W_{V_{j}} /\left(W_{V_{j}}\right)_{\nu_{0}}$, and in each step the length decreases by one. For each root $\beta_{i}$ let $m_{i}$ be such that $V_{j} \in H_{\beta_{i}, m_{i}}$, then the sequence of folds by the affine reflections $s_{\beta_{1}, m_{1}}, \ldots, s_{\beta_{q}, m_{q}}$ are all positive and, by the choice, minimal. Summarizing we have:

Lemma 11. A positively folded gallery of the same type as $\gamma_{\omega}$ is obtained from a minimal gallery by a sequence of positive folds such that each fold is minimal for the local root system associated to the corresponding vertex.

We want to compare $\sharp^{+} \delta$ and $\sharp^{+} \delta_{0}$, where $\delta$ is obtained from $\delta_{0}$ by a fold as in (11), but now assume that the positive fold is minimal.

Proposition 17. $\sharp^{+} \delta \leq \sharp^{+} \delta_{0}+\left\langle\mu-\mu_{0}, \rho\right\rangle$. Further, $\delta$ is an LS-gallery if and only if $\delta_{0}$ is an $L S$-gallery and the new fold is an LS-fold.

Since the condition of being folded by a sequence of LS-folds is equivalent to the condition for LS-paths, we get as an immediate consequence:

Corollary 2. For a fundamental coweight $\omega$ let $\pi_{\omega}:[0,1] \rightarrow X_{\mathbb{R}}^{\vee}$ be the path $t \mapsto t \omega$ and let $\pi$ be an LS-path of shape $\omega$ as in [20]. As associated gallery $\gamma_{\pi}$ in the one-skeleton of $\mathbb{A}$ take the sequence of edges and vertices lying on the path. This map $\pi \mapsto \gamma_{\pi}$ describes a bijection between the LS-paths of shape $\omega$ and the LS-galleries of the same type as $\gamma_{\omega}$.

Proof of the proposition. For $\delta_{0}=\left(\mathfrak{o}=V_{0} \subset E_{0} \supset \ldots \supset V_{r}=\mu_{0}\right)$ let $j$ be such that $\delta=\left(\mathfrak{o}=V_{0} \subset E_{0} \supset \ldots \subset V_{j} \subset s_{\beta, m}\left(E_{j}\right) \supset \ldots \supset s_{\beta, m}\left(V_{r}\right)=\mu\right)$. Denote by $\sharp_{j}^{+} \delta_{0}$ the number of positive crossings associated to the vertices $V_{k}$ for $k \geqslant j$. Since the two galleries coincide till $V_{j}$, we have $\sharp^{+} \delta-\sharp^{+} \delta_{0}=\sharp_{j}^{+} \delta-\sharp_{j}^{+} \delta_{0}$.

Let $\nu_{0}$ be the rational weight $\mu_{0}-V_{j}$ and set $\nu=\mu-V_{j}$. There exists a rational number $0<r \leq 1$ and elements $\kappa, \tau \in W / W_{\omega}$ such that $\nu_{0}=r \tau(\omega), \nu=r \kappa(\omega), r\langle\kappa(\omega), \beta\rangle \in \mathbb{Z}$, and $s_{\beta} \tau=\kappa$. Note that

$$
\begin{aligned}
\langle\omega+\mu, \rho\rangle-\left\langle\omega+\mu_{0}, \rho\right\rangle & =\left\langle\mu-\mu_{0}, \rho\right\rangle \\
& =\langle r(\kappa(\omega)-\tau(\omega)), \rho\rangle \\
& =\frac{r}{2}\left(\sum_{\gamma>0}\langle\kappa(\omega), \gamma\rangle-\sum_{\gamma>0}\langle\tau(\omega), \gamma\rangle\right) .
\end{aligned}
$$

We need the following simple lemma, which we state without proof.

Lemma 12. Let $\psi$ be a root system with Weyl group $W(\Psi)$ and let $\nu$ be a dominant weight. Fix $s_{\beta} \tau \in W(\Psi) / W(\Psi)_{\nu}$ and let $\beta$ be a positive root such that $s_{\beta} \tau<\tau$. We divide the set of positive roots into $\Psi^{+}=A \cup B$, where $A=\left\{\gamma>0 \mid s_{\beta}(\gamma)>0\right\}$ and $B=\left\{\gamma>0 \mid s_{\beta}(\gamma)<0\right\}$. Consider the following sets:

$$
\begin{array}{ll}
A_{\tau}^{+}=\{\gamma \in A \mid\langle\tau(\nu), \gamma\rangle \geq 0\} & A_{\tau}^{0}=\{\gamma \in A \mid,\langle\tau(\nu), \gamma\rangle=0\} \\
B_{\tau}^{+}=\{\gamma \in B \mid\langle\tau(\nu), \gamma\rangle \geq 0\} & B_{\tau}^{0}=\{\gamma \in B \mid\langle\tau(\nu), \gamma\rangle=0\}
\end{array}
$$

and similarly we define the sets $A_{\tau}^{-}$and $B_{\tau}^{-}$.

Then $s_{\beta}\left(A_{\tau}^{ \pm}\right)=A_{s_{\beta} \tau}^{ \pm}, s_{\beta}\left(A_{\tau}^{0}\right)=A_{s_{\beta} \tau}^{0},-s_{\beta}\left(B_{\tau}^{+}\right)=B_{s_{\beta} \tau}^{-},-s_{\beta}\left(B_{\tau}^{-}\right)=B_{s_{\beta} \tau}^{+}$and $-s_{\beta}\left(B_{\tau}^{0}\right)=$ $B_{s_{\beta} \tau}^{0}$. Further, $B_{\tau}^{+} \cup\{\beta\} \subset B_{s_{\beta} \tau}^{+}$, and one has equality if and only if $\ell(\tau)=\ell\left(s_{\beta} \tau\right)+1$ for the length function $\ell$ on $W(\Psi) / W(\Psi)_{\nu}$. 
Using the notation and the results of Lemma 12, this sums reduces to

$$
\langle\omega+\mu, \rho\rangle-\left\langle\omega+\mu_{0}, \rho\right\rangle=r\left(\sum_{\gamma \in B_{\kappa}^{+}}\langle\kappa(\omega), \gamma\rangle-\sum_{\gamma \in B_{\tau}^{+}}\langle\tau(\omega), \gamma\rangle\right),
$$

since $\langle\kappa(\omega), \gamma\rangle=\left\langle s_{\beta} \kappa(\omega), s_{\beta}(\gamma)\right\rangle=\left\langle\tau(\omega), s_{\beta}(\gamma)\right\rangle$. Again by Lemma 12, we can divide $B_{\kappa}^{+}$ into $B_{\tau}^{+} \cup\{\beta\} \cup$ Rest and get:

$$
\begin{array}{r}
\langle\omega+\mu, \rho\rangle-\left\langle\omega+\mu_{0}, \rho\right\rangle= \\
=r\left(\sum_{\gamma \in B_{\tau}^{+}}\langle\kappa(\omega)-\tau(\omega), \gamma\rangle+\langle\kappa(\omega), \beta\rangle\right. \\
\left.+\sum_{\gamma \in \operatorname{Rest}}\langle\kappa(\omega), \gamma\rangle\right) \\
\quad \sum_{\gamma \in B_{\tau}^{+}} r\langle\kappa(\omega), \beta\rangle\langle\beta, \gamma\rangle+r\langle\kappa(\omega), \beta\rangle \\
+\sum_{\gamma \in \operatorname{Rest}} r\langle\kappa(\omega), \gamma\rangle .
\end{array}
$$

We want to compare this sum to $\sharp_{j}^{+} \delta-\sharp_{j}^{+} \delta_{0}$. If $\gamma$ is a positive root, then $\left(V_{k}, E_{k}\right)$ crosses some wall $H_{\gamma, p}$ positively for some $k \geqslant j$ only if $\langle\tau(\omega), \gamma\rangle>0$, and if $\gamma \in \Phi_{V_{j}}$, then the number of such crossings is $r\langle\tau(\omega), \gamma\rangle$. If $\gamma \notin \Phi_{V_{j}}$, then the number of such crossings is $\lfloor r\langle\tau(\omega), \gamma\rangle\rfloor$, the largest integer smaller or equal to $r\langle\tau(\omega), \gamma\rangle$. So again with the notation as in Lemma 12 and the decomposition $B_{\kappa}^{+}=B_{\tau}^{+} \cup\{\beta\} \cup$ Rest:

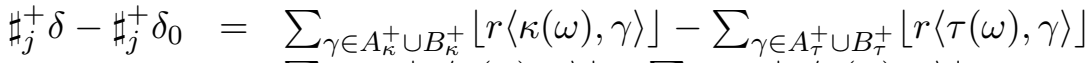

$$
\begin{aligned}
& =\sum_{\gamma \in B_{\kappa}^{+}}\lfloor r\langle\kappa(\omega), \gamma\rangle\rfloor-\sum_{\gamma \in B_{\tau}^{+}}\lfloor r\langle\tau(\omega), \gamma\rangle\rfloor \\
& =\sum_{\gamma \in B_{\tau}^{+}}(\lfloor r\langle\kappa(\omega), \gamma\rangle\rfloor-\lfloor r\langle\tau(\omega), \gamma\rangle\rfloor)+\lfloor r\langle\kappa(\omega), \beta\rangle\rfloor \\
& +\sum_{\gamma \in \operatorname{Rest}}\lfloor r\langle\kappa(\omega), \gamma\rangle\rfloor \\
& =\sum_{\gamma \in B_{\tau}^{+}}(\lfloor r\langle\kappa(\omega), \gamma\rangle\rfloor-\lfloor r\langle\kappa(\omega), \gamma\rangle-r\langle\kappa(\omega), \beta\rangle\langle\beta, \gamma\rangle\rfloor) \\
& +\lfloor r\langle\kappa(\omega), \beta\rangle\rfloor+\sum_{\gamma \in \text { Rest }}\lfloor r\langle\kappa(\omega), \gamma\rangle\rfloor
\end{aligned}
$$

Since $r\langle\kappa(\omega), \gamma\rangle$ is an integer by assumption, we obtain:

$$
\sharp_{j}^{+} \delta-\sharp_{j}^{+} \delta_{0}=\sum_{\gamma \in B_{\tau}^{+}} r\langle\kappa(\omega), \beta\rangle\langle\beta, \gamma\rangle+r\langle\kappa(\omega), \beta\rangle+\sum_{\gamma \in \operatorname{Rest}}\lfloor r\langle\kappa(\omega), \gamma\rangle\rfloor
$$

As a consequence we see:

$$
\begin{aligned}
\left(\left\langle\mu-\mu_{0}, \rho\right\rangle\right)-\left(\sharp^{+} \delta-\sharp^{+} \delta_{0}\right) & =\left(\langle\omega+\mu, \rho\rangle-\left\langle\omega+\mu_{0}, \rho\right\rangle\right)-\left(\sharp_{j}^{+} \delta-\sharp_{j}^{+} \delta_{0}\right) \\
& =\sum_{\gamma \in \text { Rest }}(r\langle\kappa(\omega), \gamma\rangle-\lfloor r\langle\kappa(\omega), \gamma\rangle\rfloor),
\end{aligned}
$$

which proves the inequality in the proposition. We have equality if and only if the right hand term above is zero. The target $\mu$ is a special point, so $r\langle\kappa(\omega), \gamma\rangle$ is an integer if an only if $\gamma \in \Phi_{V_{j}}$. Since the folding is minimal by assumption, the intersection Rest $\cap \Phi_{V_{j}}=\emptyset$. But this implies that we have equality if and only if Rest $=\emptyset$, i.e., the fold is an LS-fold by Lemma 12. In particular, $\delta$ is an LS-gallery if and only if $\delta_{0}$ is an LS-gallery and the new fold is an LS-fold.

\subsection{Connection with the path model}

Summarizing the results above, we have the following connection between the path model of a representation and the one-skeleton galleries: 
Corollary 3. Write a dominant coweight $\lambda=\omega_{i_{1}}+\ldots+\omega_{i_{r}}$ as a sum of fundamental coweights, write $\underline{\lambda}$ for this ordered decomposition. Let $\mathcal{P}_{\underline{\lambda}}$ be the associated path model of LS-paths of shape $\underline{\lambda}$ defined in [20]. The associated one-skeleton galleries (same procedure as in Corollary 2) are precisely the LS-galleries of the same type as $\gamma_{\omega_{i_{1}}} * \ldots * \gamma_{\omega_{i_{r}}}$.

In fact, the notion of a defining chain for LS-paths introduced by Lakshmibai, Musili and Seshadri coincides in this case with the notion of a defining chain for the associated gallery. As an immediate consequence of Theorem 2 and Proposition 16 we get the following character formula. In combination with Corollary 3, this provides a geometric proof of the path character formula, first conjectured by Lakshmibai (see for example [17]) and proved in 20]:

Corollary 4. Char $V(\lambda)=\sum_{\delta} e^{\text {target }(\delta)}$, where the sum runs over all LS-galleries of the same type as $\gamma_{\lambda}$.

Proof. The formula in Theorem 2 and the results above show that the highest power of $q$ in the Laurent polynomial $L_{\lambda, \mu}$ is $\langle\lambda+\mu, \rho\rangle$, and the coefficient of the highest power is the number of LS-galleries having $\mu$ as a target. Since $P_{\lambda} \rightarrow s_{\lambda}$ for $q \rightarrow \infty$, this proves the character formula.

Let us now consider some of the special cases discussed in section 5.4, these cases occur already in [14]. The question why for some enumeration of the fundamental weights the combinatorics for tableaux becomes suddenly much easier than for other enumerations seems to have a geometric answer: because for special orderings locally minimal and globally minimal are equivalent conditions for one-skeleton galleries.

\subsection{LS-tableaux and LS-galleries}

It remains to describe the semi-standard tableaux corresponding to LS-galleries, we call these LS-tableaux. Since the condition of being folded by a sequence of LS-folds is equivalent to the condition for LS-paths, these tableaux can be found in [21], we refer here to a slightly different but equivalent description by Lakshmibai.

Proposition 18. i) In type $\mathrm{A}_{n}$ all semistandard tableaux are LS-tableaux.

ii) In type $\mathrm{B}_{n}$ a semistandard tableau $\mathcal{T}$ is an LS-tableau if and only if the following holds: each pair of columns $\left(C_{1}, C_{2}\right)$ corresponding to a gallery for a non-minuscule weight satisfies the conditions for an admissible pair in Proposition B1 of [15].

iii) In type $\mathrm{C}_{n}$ a semistandard tableau $\mathcal{T}$ is an LS-tableau if and only if the following holds: each pair of columns $\left(C_{1}, C_{2}\right)$ corresponding to a gallery for a non-minuscule weight satisfies the conditions for an admissible pair in Proposition C1 of [15].

Example 10. The tableau of type $\mathrm{B}_{3}$ in Example 5 is semistandard but not $L S$.

Acknowlegments. We would like to thank Venkatraman Lakshmibai for helping us improving the manuscript. The first author would also like to thank Guy Rousseau for very useful comments and acknowledge financial support by the ANR as member of the project ANR-09-JCJC-0102-01. The second author acknowledge also financial support by the priority program SPP 1388 of the DFG. Both authors thank the Hausdorff research Institute 
for Mathematics for the hospitality during the Trimester Program "On the interaction of representation theory with geometry and combinatorics".

\section{References}

[1] P. Baumann and S. Gaussent, On Mirković-Vilonen cycles and crystal combinatorics, Represent. Theory 12 (2008), pp. 83-130.

[2] S. Billey and V. Lakshmibai, Singular Loci of Schubert varieties, Birkhuser, Progress in Math. 182.

[3] N. Bourbaki, Éléments de mathématique. Fasc. XXXIV. Groupes et algèbres de Lie. Chapitres IV, V, VI, Actualités Scientifiques et Industrielles, No. 1337, Hermann, Paris, 1968.

[4] K. S. Brown, Buildings, Springer-Verlag, New-York (1989).

[5] F. Bruhat and J. Tits, Groupes réductifs sur un corps local, I, Publ. Math. I.H.E.S., 41, (1972), pp. 5-252.

[6] F. Bruhat and J. Tits, Groupes réductifs sur un corps local, II, Publ. Math. I.H.E.S., 60, (1984), pp. 5-184.

[7] C. Contou-Carrère, Géométrie des groupes semi-simples, résolutions équivariantes et lieu singulier de leurs variétés de Schubert, Thèse d'état, (1983), Université Montpellier II.

[8] V. Deodhar, On some geometric aspects of Bruhat orderings. I. A finer decomposition of Bruhat cells, Inv. Math. 79, (1985), pp 499-511.

[9] S. Gaussent, P. Littelmann, LS-galleries, the path model, and MV-cycles, Duke Math. J. 127, (2005), pp. 35-88.

[10] M. Kapovich, J. Millson, A path model for geodesics in euclidean buildings and its applications to representation theory, Geometry, Groups and Dynamics 2, (2008), pp. 405-480.

[11] T. Hausel, F. Rodriguez-Villegas, Mixed Hodge polynomials of character varieties. With an appendix by Nicholas M. Katz, Invent. Math. 174 (2008), no. 3, pp. 555-624.

[12] I. Klostermann, Generalization of the Macdonald formula for Hall-Littlewood polynomials, preprint, arXiv:1102.0571.

[13] S. Kumar, Kac-Moody Groups, their Flag Varieties and Representation Theory, Birkhäuser, (2002).

[14] V. Lakshmibai, C. Musili, C. S. Seshadri, Geometry of G/P IV. Standard monomial theory for classical types, Proc. Indian Acad. Sci. Sect. A Math. Sci. 88 (1979), no. 4, pp. 279-362.

[15] V. Lakshmibai, Bases pour les reprsentations fondamentales des groupes classiques. I., C. R. Acad. Sci. Paris Sér. I Math. 302, 1986, no. 10, pp 387-390. 
[16] V. Lakshmibai, C.S. Seshadri, Geometry of $G / P \quad V$, J. Algebra 100 (1986), pp. $462-557$.

[17] V. Lakshmibai, C. S. Seshadri, Standard monomial theory, Proceedings of the Hyderabad Conference on Algebraic Groups, Manoj Prakashan, Madras, (1991), pp. 279-323.

[18] C. Lenart, Hall-Littlewood polynomials, alcove walks, and fillings of Young diagrams, I, Discrete Math., 311 : 4 (2011), 258-275.

[19] C. Lenart, Haglund-Haiman-Loehr type formulas for Hall-Littlewood polynomials of type B and C, Algebra Number Theory, 4 : 7 (2010).

[20] P. Littelmann, A Littlewood-Richardson rule for symmetrizable Kac-Moody algebras, Invent. Math. 116 (1994), pp. 499-525.

[21] P. Littelmann, A generalization of the Littlewood-Richardson rule, J. Algebra 130 (1990), pp. 328-368.

[22] P. Littelmann, Contracting modules and Standard Monomial Theory for symmetrizable Kac-Moody algebras, J. Amer. Math. Soc. 11, Number 3, (1998), pp. 551-567.

[23] I. G. Macdonald, Symmetric functions and Hall polynomials, Oxford Mathematical Monographs. The Clarendon Press Oxford University Press, New York, second edition, (1995).

[24] B. C. Ngô, P. Polo, Résolutions de Demazure affines et formule de Casselman-Shalika géométrique, J. Algebraic Geom. 10 (2001), no. 3, pp. 515-547.

[25] M. Ronan, Lectures on Buildings, Academic Press, (1989).

[26] C. Schwer, Galleries, Hall-Littlewood polynomials and structure constants of the spherical Hecke algebra, Int. Math. Res. Not. 2006, Art. ID 75395, 31 pp.

[27] J. Tits, Uniqueness and Presentation of Kac-Moody Groups over Fields, J. of Algebra, 105, (1987), pp. 542-573.

[28] J. Tits, Résumé de cours, Annuaire du Collège de France, Paris, (1982), pp. 91-106.

[29] J. Tits, Buildings of spherical type and finite BN-pairs, Lecture notes in Math. 386, Springer-Verlag, Heidelberg (1974). 